\title{
A CRITICAL ANALYSIS OF VULNERABLE POPULATIONS IN THE DURHAM-YORK ENERGY-FROM- WASTE INCINERATOR HUMAN HEALTH RISK ASSESSMENT
}

by

\section{Nazira Panchbhaya}

Honours Bachelor of Science, University of Toronto, 2011

\author{
A thesis \\ presented to Ryerson University \\ in partial fulfillment of the \\ requirements for the degree of \\ Master of Applied Science \\ in the Program of \\ Environmental Applied Science and Management
}

Toronto, Ontario, Canada, 2015

(C) Nazira Panchbhaya, 2015 


\section{AUTHOR'S DECLARATION}

I hereby declare that I am the sole author of this thesis. This is a true copy of the thesis, including any required final revisions, as accepted by my examiners.

I authorize Ryerson University to lend this thesis to other institutions or individuals for the purpose of scholarly research.

I further authorize Ryerson University to reproduce this thesis by photocopying or by other means, in total or in part, at the request of other institutions or individuals for the purpose of scholarly research.

I understand that my thesis may be made electronically available to the public

Nazira Panchbhaya 


\begin{abstract}
A Critical Analysis of Vulnerable Populations in the Durham-York Energy-From-Waste Incinerator Human Health Risk Assessment
\end{abstract}

Nazira Panchbhaya

Master of Applied Science

Environmental Applied Science and Management

2015

Ryerson University

A health risk assessment is an essential tool to assess effects of polluting facilities on vulnerable populations. The Durham-York Energy Centre is the first incinerator built in Ontario in over 20 years and it has caused public controversy due to the health effects of such facilities. The proponents conducted a human health risk assessment (HHRA) according to best practices to effectively protect the public. The objective of this thesis is to establish a HHRA best practice framework for vulnerable populations, particularly pregnant women and fetuses, to confirm if best practices were achieved and determine whether this group was adequately considered in the HHRA. Analysis ultimately showed that the Durham-York HHRA complied with most best practices but the failure to identify pregnant women and fetuses as a vulnerable population lead to some important deficiencies and omissions in the assessment. 


\section{ACKNOWLEDGEMENTS}

There were many individuals that I would like to recognize, as without their consistent guidance and support, the completion of this thesis would not have been possible.

First and foremost, I would like to thank Dr. Ron Pushchak for his constant support and guidance throughout this thesis. He invested his valuable time in me, making even the most daunting tasks seem possible. His constant motivation resulted in the completion of this thesis.

A thank you to Ryerson University for providing me with the funding for my Master's degree.

I would also like to thank all the experts who participated in my research and will remain anonymous for ethical reasons.

My graduating class at Ryerson made the entire process educational, practical and interesting and I would like to thank them all.

A big thank you to Professor Lynda McCarthy for her invaluable critiquing and honesty.

I owe a great thank you to my parents, siblings, my in-laws and the rest of my family members for providing endless support, love and motivation to complete this thesis. This would definitely not have been possible without you all.

And of course, I am thankful to my husband for motivating me when I was struggling and providing his love and being my rock when I felt lost. 
TABLE OF CONTENTS

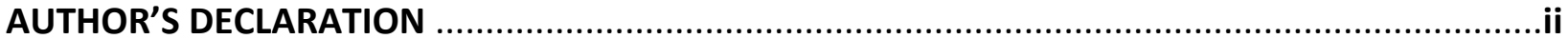

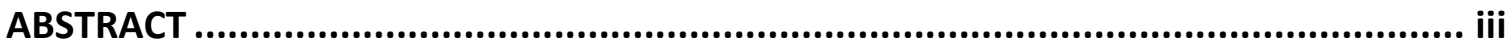

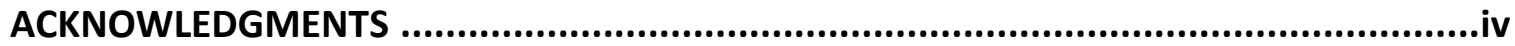

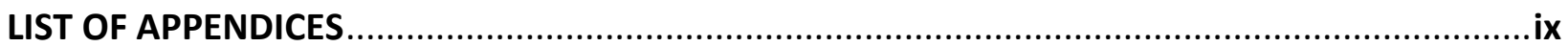

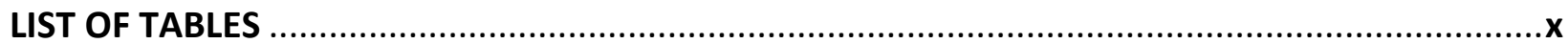

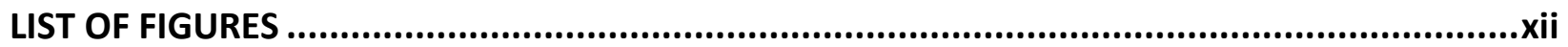

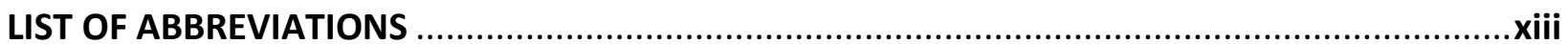

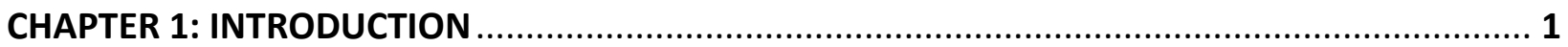

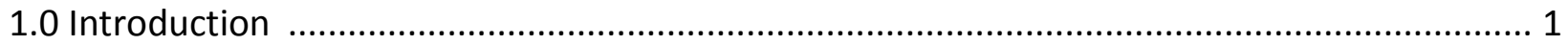

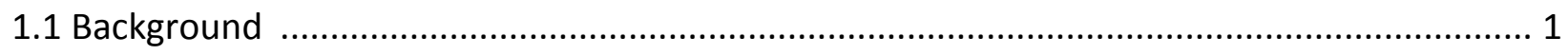

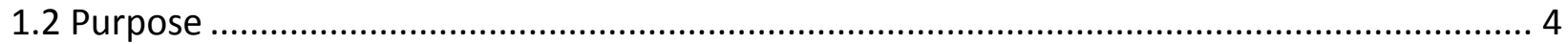

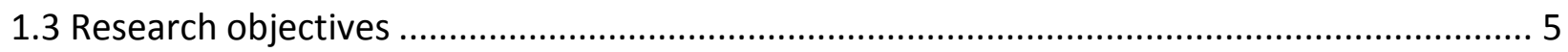

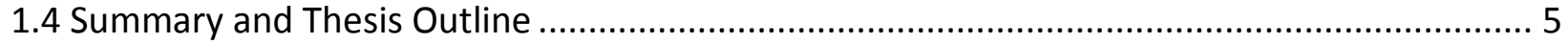

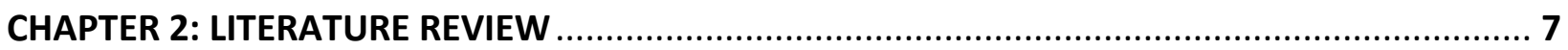

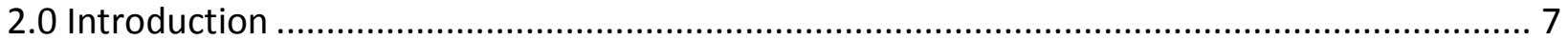


2.1 Energy-From-Waste Facilities: A General Overview

2.2 The Durham-York Environmental Assessment Process.................................................... 8

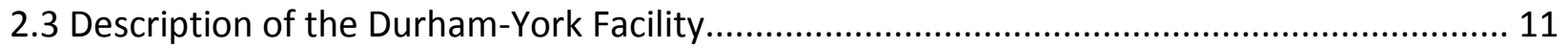

2.3.1 Air Pollution Control ................................................................................... 12

2.4 Contaminants of Potential Concern and Potential Health Effects .................................. 15

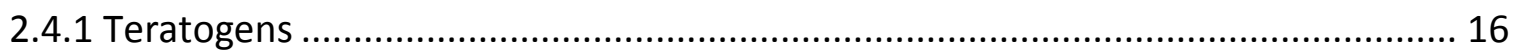

2.4.2 Contaminants of Potential Concern and their Associated Health Effects .............. 17

2.4.2 Emission Exceedances for Facilities with Modern Air Pollution Control Technologies

2.5 Susceptible Populations of Focus: Pregnant Women and Fetuses .................................. 26

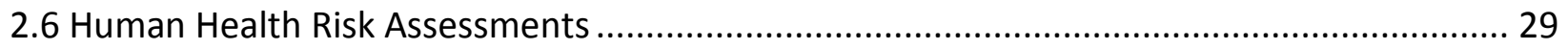

2.6.1 Human Health Risk Assessment for Pregnant Women and Fetuses ..................... 32

2.6.2 Inaccuracies of Human Health Risk Assessments ............................................ 35

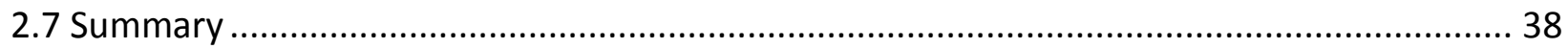

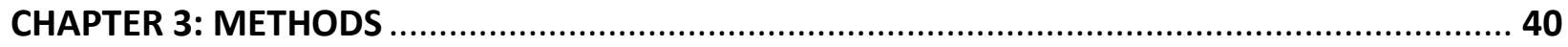

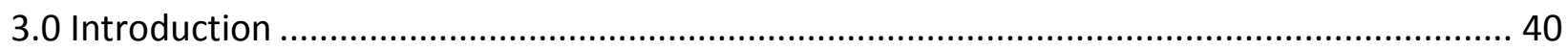

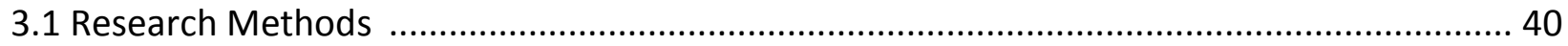

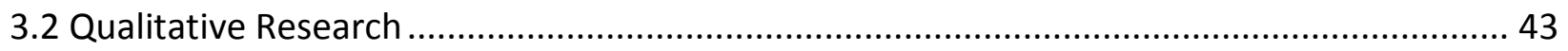


3.2.1 Interview Template

3.2.2 Recruiting Process

3.2.3 Participation Rates

3.3.1 Problem Formulation/Screening

3.3.2 Hazard Identification 60

3.3.3 Exposure Assessment 63

3.3.4 Dose-Response Assessment

3.3.5 Risk Characterization

3.4 Evaluation of the Durham-York Human Health Risk Assessment to the Best Practice Criteria

4.1.2 Problem Formulation/Screening: Deficiencies and Absences of Best Practice Criteria. 
4.2 Hazard Identification: Conformities and Deficiencies

4.2.1 Hazard Identification: Conformities with Best Practice Criteria. 86

4.2.2 Hazard Identification: Deficiencies and Absences of Best Practice Criteria 88

4.3 Exposure Assessment: Conformities and Deficiencies 89

4.3.1 Exposure Assessment: Conformities with Best Practice Criteria 89

4.3.2 Exposure Assessment: Deficiencies and Absences of Best Practice Criteria 93

4.4 Dose-Response Assessment: Conformities and Deficiencies 94

4.4.1 Dose-Response Assessment: Conformities with Best Practice Criteria 95

4.4.2 Dose-Response Assessment: Deficiencies of Best Practice Criteria 99

4.5 Risk Characterization: Conformities and Deficiencies 100

4.5.1 Risk Characterization: Conformities with Best Practice Criteria 101

4.5.2 Risk Characterization: Deficiencies and Absences of Best Practice Criteria 106

4.6 Summary 108

CHAPTER 5: CONCLUSIONS .109

5.0 Introduction 109

5.1 The Complete Evaluation of the Durham-York Human Health Risk Assessment and its Compliance to Best Practices 109

5.2 Summary of the Research Methods and Major Findings 115 


\section{LIST OF APPENDICES}

Appendix A: Research Consent Form

Appendix B: Letter of Invitation

Appendix C: Interview Template

Appendix D: Human Health Risk Assessment Best Practice Framework with Emphasis on 


\section{LIST OF TABLES}

Table 3.1: A summary of the Problem Formulation/Screening best practice criteria 59

Table 3.2: A summary of the hazard identification best practice criteria

Table 3.3: A summary of the exposure assessment best practice criteria...... 66

Table 3.4: A summary of the Dose-Response best practice criteria 71

Table 3.5: A summary of the Risk Characterization best practice criteria 76

Table 4.1: Criteria that conformed with best practices in the Problem Formulation/Screening stage of the Durham-York human health risk assessment 80

Table 4.2 Deficiencies of best practice criteria in the Problem Formulation/Screening stage of the Durham-York human health risk assessment

Table 4.3: Absence of a best practice criterion in the Problem Formulation/Screening stage of the Durham-York human health risk assessment 80

Table 4.4: Criteria that conformed with best practices in the Hazard Identification stage of the Durham-York human health risk assessment 87

Table 4.5: Deficiency of a best practice criterion in the Hazard Identification stage of the Durham-York human health risk assessment

Table 4.6: Absence of a best practice criterion in the Hazard Identification stage of the DurhamYork human health risk assessment

Table 4.7: Criteria that conformed with best practices in the Exposure Assessment of the Durham-York human health risk assessment 90 
Table 4.8: Deficiencies of best practice criteria in the Exposure Assessment of the Durham-York human health risk assessment 90

Table 4.9: Absence of a best practice criterion in the Exposure Assessment of the Durham-York human health risk assessment 90

Table 4.10: Criteria that conformed with best practices in the Dose-Response Assessment of the Durham-York human health risk assessment 95

Table 4.11:Deficiencies of best practice criteria in the Dose-Response Assessment of the Durham-York human health risk assessment 95

Table 4.12: Criteria that conformed to best practices in the Risk Characterization stage of the Durham-York human health risk assessment 101

Table 4.13: Deficiencies of best practice criteria in the Risk Characterization stage of the Durham-York human health risk assessment 101

Table 4.14: Absence of best practice criterion in the Risk Characterization stage of the DurhamYork human health risk assessment 101

Table 5.1 :The results of the Durham-York human health risk assessment when evaluated against the best practice framework

Table 5.2 The conformities, deficiencies and absences of criteria in the best practice framework by the Durham-York human health risk assessment that pertained directly to vulnerable populations 115 


\section{LIST OF FIGURES}

Figure 2.1: Overview of the Durham-York Environmental Assessment Process

Figure 3.1: Methods used to achieve research objectives

Figure 3.2: Number and specialization of total individuals contacted...................................... 50

Figure 3.3: Number and specialization of participants ......................................................... 50 


\section{LIST OF ABBREVIATIONS}

AENV: Alberta Environment

AERMOD: American Meteorological Society / Environmental Protection Agency Regulatory

Model

APC: Air Pollution Control

As: Arsenic

ATSDR: Agency for Toxic Substances and Disease Registry

CalEPA: California Environmental Protection Agency

CALPUFF: California Puff Model

CCME: Canadian Council of the Ministers of the Environment

Cd: Cadmium

$\mathrm{CH}_{4}$ : Methane

CO: Carbon Monoxide

$\mathrm{CO}_{2}$ : Carbon Dioxide

C of A-Certificate of Approval

COPC: Contaminants of Potential Concern

Cr: Chromium

CR: Concentration Ratio 
EA: Environmental Assessment

EAA: Environmental Assessment Act

EfW: Energy-from-Waste

EIA: Environmental Impact Assessment

EIS: Environmental Impact Statement

ESDM: Emissions Summary and Dispersion Modelling

HHRA- Human Health Risk Assessment

HIA: Health Impact Assessment

HCl: Hydrogen Chloride

HF: Hydrogen Fluoride

Hg: Mercury

HQ: Hazard Quotient

ILCR: Incremental Lifetime Cancer Risk

ILSI: International Life Sciences Institute

IRIS: US EPA Integrated Risk Information System

LADD: Lifetime Average Daily Dose

LCR: Lifetime Cancer Risk 
MSW: Municipal Solid Waste

MOE: Ministry of the Environment

NDP: New Democratic Party

$\mathbf{N H}_{3}$ : Ammonia

NO : Nitrogen Oxides

PAH: Polycyclic Aromatic Hydrocarbons

Pb: Lead

PCB: Polychlorinated Biphenyls

PCP: Pentachlorophenol

PCDD: Polychlorinated Dibenzodioxins

PCDF: Polychlorinated Dibenzofurans

PM: Particulate Matter

POP: Persistent Organic Pollutants

RfD: Reference Dose

RfC: Reference Concentration

SF: Cancer Slope Factor

SO ${ }_{x}$ : Sulfur Dioxides 
SNCR: Selective Non-Catalytic Reduction

TCEQ: Texas Commission of Environmental Quality

TPY: Tonnes Per Year

TRVs: Toxicity Reference Values

UK: United Kingdom

UR: Unit Risk

USEPA: United States Environmental Protection Agency

VOCs: Volatile Organic Compounds

WHO: World Health Organization 


\section{CHAPTER 1: INTRODUCTION}

\subsection{Introduction}

It has been over 20 years since Ontario has built its last energy-from-waste (EfW) facility, which is why concerns are raised with the construction and development of the Durham-York EfW facility in Clarington. Incinerators raise significant health concerns amongst members of the community and for this reason, a human health risk assessment $(\mathrm{HHRA})^{1}$ was conducted. Although this assessment was set out to evaluate any public health concerns, questions remain on how well the HHRA addressed specific concerns in vulnerable population segments such as pregnant women and fetuses.

\subsection{Background}

Currently, Ontario has only one incinerator processing municipal solid waste (MSW) operating in the Regional Municipality of Peel. The facility was built prior to the 1992 ban on new incineration projects which was in place to eliminate the need for constant fuel, limiting the overall reduction of waste (Carter-Whitney, 2007). At this time, there was also an elimination of numerous types of incinerators in Ontario, as many were not meeting air emission standards (Ron Pushchak per. comm., 2013). The moratorium was lifted in 1995 (Ontario Ministry of the Environment, 1999) but due to lingering public concerns and high costs

\footnotetext{
${ }^{1}$ A human health risk assessment is a document that estimates the nature and the probability of adverse health effects when humans are exposed to contaminants of concern (United States Environmental Protection Agency, 2012a)
} 
to building new facilities, the construction of incinerators was at a halt. Therefore, municipalities continued to rely heavily on landfills for waste disposal (Jackson, 1999). The problem is rapidly growing with landfills reaching capacity, siting difficulties and stringent requirements laid out in Ontario Regulation 232/98- Landfilling Sites. As a result, incinerators are gaining more attention in Ontario (Carter-Whitney, 2007).

The Regions of Durham and York have committed significant resources identifying solutions for long-term waste disposal. From 1974 to the mid-1990s, seven small municipal landfills closed in Durham Region, forcing them to ship their waste to the Keele Valley landfill in Vaughan (Januszkiewicz, 2009). In 2002, with the aforementioned landfill closing, Durham Region entered an agreement with the Pine Tree Acres landfill in Michigan (Januszkiewicz, 2009). With Michigan announcing its initiative to close the border to Ontario waste in 2004 (Januszkiewicz, 2009) and failed attempts in constructing new landfill facilities, the Region was forced to seek an alternate solution to their waste disposal problem (York Region, 2011). In 2005, Durham and York established a partnership to seek long-term solutions to manage postdiversion residual waste (Environmental Services Committee, 2013). In 2010, an Environmental Assessment approval was issued for the construction of a state- of-the-art mass burn incinerator predicted to process 140,000 tonnes of waste per year with a maximum design capacity of 400,000 tonnes per year (tpy) (Ollson, Knopper, Whitfield Aslund et al., 2014) while generating renewable energy ${ }^{2}$ (York Region,2011). During the same year, Michigan closed the

\footnotetext{
${ }^{2}$ The facility is expected to generate about 14 megawatts of renewable net electric energy on a continuous basis (Durham York Waste, 2015)
} 
border to Ontario's municipal waste (Januszkiewicz, 2009) which further attested the need for a waste management solution.

The facility has technologically modern air pollution control (APC) technology and will manage a considerable amount of waste, however, there still remain public concerns of the potential health risks associated with incineration, particularly due to the emissions from the combustion process ${ }^{3}$. Requirements to operate an incineration facility have evolved dramatically since the 1992 ban, with changes in APC technology and stringent air pollution limits outlined in Ontario Regulation 419/05: Air Pollution-Local Air Quality. The approval of this incinerator will trigger an increase in incineration projects in future years as a solution to waste management problems-ENTECH-REM is also proposing a similar facility in Port Hope but with significant controversy. Therefore, it is imperative to examine the HHRA conducted for the Durham-York facility to ensure that all factors are adequately addressed and follow best practices $^{4}$ of incineration HHRAs, particularly the vulnerable sub-population of pregnant women and fetuses. This research can guide future projects to ensure that all significant practices for a thorough HHRA are identified for the aforementioned population. This study will critically examine the literature and previous HHRAs to create a HHRA best practice framework that will aid in determining whether vulnerable populations particularly fetuses and pregnant women were adequately considered in the Durham-York HHRA.

\footnotetext{
${ }^{3}$ The combustion process is associated with the release of contaminants into the air due to the burning of waste and the release of chemicals is what raises questions (Allsopp et al., 2001).

${ }^{4}$ According to Storey and Noble (2004), a best practice is essentially the best way of doing things and is about selecting the highest quality option or set of procedures that are effective and produce a desired outcome. For the context of this research, a best practice represents the practice that had the highest number of occurrences in different sections of an HHRA, by conducting a literature review and review of previously conducted HHRAs and practices that were thought to be an important inclusion in the HHRA according to literature.
} 


\subsection{Purpose}

The HHRA for the Durham-York facility followed best practices as per the proponents (Jacques Whitford Limited, 2007). This thesis has critically examined the HHRA for the DurhamYork mass burn incinerator with a goal of determining whether human health risks were appropriately considered, specifically focusing on the vulnerable population of pregnant woman and fetuses.

There were a few key reasons why pregnant women and fetuses were chosen to be the focus of this research. Firstly, these two population segments and their health risks have not been addressed sufficiently in the literature while ample research surrounds healthy adults and post-natal children. Pregnant women and fetuses experience developing and physiological changes that react differently to exposures compared to other sub-populations. A detailed discussion with supporting literature outlining the vulnerabilities of this group will be presented in section 2.6.1 of Chapter 2. Secondly, several studies have outlined the effects of contaminants on pregnant women and their developing fetus, which is detailed in section 2.4.1 in Chapter 2. Furthermore, the peer review of the Durham-York HHRA stated that the risk assessment was conducted in a satisfactory manner but it failed to mention why the vulnerabilities of pregnant women and fetuses and other sensitive populations were not mentioned in the HHRA (Smith and Wilson, 2009). For these reasons, this sub-population was of interest and is of key focus in this study. 


\subsection{Research Objectives}

The goal of this thesis is to create a HHRA framework specific for incineration projects in Ontario and to use it to evaluate the Durham-York EfW incinerator HHRA. This thesis explores the fundamental question:

Did the Durham-York EfW incinerator HHRA conform to best practices and did it adequately consider health risks of vulnerable populations specifically pregnant women and fetuses?

There are four essential objectives that were achieved in order to answer the preceding research question:

1. Established HHRA best practices for incineration projects from authoritative sources, focusing on pregnant women and their fetuses

2. The best practices identified in literature, existing HHRAs for incineration projects and from authoritative experts was used to create a HHRA framework in form of a checklist

3. After the framework was created, it was used to evaluate the Durham-York HHRA

4. This helped in determining if the HHRA followed best practices and to the extent it followed best practices (conformities, deficiencies and absences) and if it adequately considered pregnant women and fetuses

\subsection{Summary and Thesis Outline}

Overall, this chapter shed insight into the research question that this study aims to address. Background information was presented on the Durham-York facility as well as related 
concerns. The purpose was identified along with the research objectives which will set the foundation for the rest of the study. In the following chapter, a detailed literature review will provide the necessary platform to support the research question. It will highlight the main concerns of incinerators and will provide literature supporting why pregnant women and fetuses should be considered as a vulnerable population in HHRAs. Inadequacies relating to risk assessments will also be highlighted. Chapter 3 will outline the research methods that resulted in the best practice framework. Chapter 4 will present the evaluation results of the DurhamYork HHRA. Chapter 5 will provide a discussion of the results, major findings as well as potential applications and future research initiatives of this study. 


\section{CHAPTER 2: LITERATURE REVIEW}

\subsection{Introduction}

The chapter provides a brief overview of EfW facilities as well as the EA process the Regions underwent to select the preferred option of managing waste. Following this, the Durham-York EfW facility will be described, including its APC technology. The chapter will also discuss the contaminants of potential concern (COPC) that are emitted by incinerators and their potential health effects; a discussion of pregnant women and their fetuses and HHRAs in general and for pregnant women and fetuses. It will conclude with a discussion of studies that highlight the inadequacies of HHRAs.

\subsection{Energy-From-Waste Facilities: A General Overview}

Incineration facilities that produce energy in the process are known as EfW facilities or thermal treatment facilities. This disposal method of waste involves the combustion of materials at higher temperatures after the diversion of glass, paper, plastic and other recyclable materials as well as the separation of organics (Cucchiella, D' Adamo and Gastaldi, 2014; Demirbas, 2011). The ash that is produced at the end of the combustion process is then disposed of in a landfill. The combustion process is used to generate thermal energy as a secondary product (Cucchiella, D' Adamo and Gastaldi, et al., 2014). The incineration process is guided under the paradigm of Reduce, Reuse, Recycle and Recover (Giroux Environmental Consulting, 2014). Therefore, EfW is suggested as an effective method not only managing large volumes of waste but also may serve as a cleaner source of energy compared to traditional 
power generation plants relying on fossil fuels (Statistics Canada, 2012). In order to reduce potential health effects associated with emissions from EfW, major APCs are installed.

\subsection{The Durham-York Environmental Assessment Process}

An EA study assesses the potential environmental effects of an infrastructure proposal. The key components of an EA include "consultation with government agencies and the public; consideration and evaluation of alternatives and the management of potential environmental effects" (Ontario Ministry of the Environment, 2014a). All public sector projects including waste management proposals designated by the Ministry of the Environment (MOE) that have the potential for harm or significant health or environmental effects are subject to the Environmental Assessment Act (EAA) (MacViro Consultants Inc. and Jacques Whitford Limited, 2005).

The decision to construct an EfW facility was based on the EA residual waste study that Durham and York agreed to participate in. On June 30, 2005 at the completion of the study, it was decided that the thermal treatment facility was the preferred solution to managing the post-diversion residual waste in Clarington (Jacques Whitford Limited, 2009a). Although Durham and York were in a partnership in this process, Durham has a larger share of the facility than York $-78.6 \%$ compared to York's $21.4 \%$ contribution (Januszkiewicz, 2013).

The Durham-York facility specifically underwent an individual EA. It is a process required for very complex infrastructure projects in Ontario under the EAA for MSW disposal undertakings that have the potential for causing significant environmental effects (Ontario 
Ministry of the Environment, 2015). The EA planning period was from 2011 to 2045, a total of 35 years and was for the purpose of physically, biologically and/ or thermally processing waste that remains after waste diversion programs in the two Regions, to recover materials and energy and to minimize the amount of material that would ultimately be disposed of in a landfill (Ontario Ministry of the Environment, 2014).

Four essential steps guided the EA process of the facility, with each step branching off to several others. The entire EA process is outlined in Figure 2.1 below. The first step was to develop and complete the EA Terms of Reference (ToR), which established the study scope. It described how the Regions of Durham and York would meet the requirements of the EAA (Jacques Whitford Limited, 2009a). The ToR identified the purpose of the study as well as the study area, study periods and consultation to be included in the project (Ontario Ministry of the Environment, 2014b; Jacques Whitford Limited, 2009a). It also outlined the waste to be managed by the decided undertaking. Further detail on this can be found in the Durham/York Residual Waste Study-Environmental Assessment Study Document (Jacques Whitford Limited, 2009a). The ToR was developed based on consultation with the public and was approved in March of 2006 by the Ontario Minister of the Environment (Jacques Whitford Limited, 2009a). The ToR also specified the need for the site-specific HHRA along with other technical reports, which will be discussed later in this document.

The second step for the EA process was to evaluate the alternatives for the undertaking. This included evaluating mechanical treatment, biological treatment and thermal treatment (which includes combustion, gasification and pyrolysis) (Jacques Whitford Limited, 2009a). Seven steps were taken to evaluate and compare each alternative to another, in order to 
identify the best solution for the waste management problem that was present in Durham and York. The Durham-York EA presents a detailed list of the steps. The result of this process was the identification of an EfW thermal treatment facility. The third step in the EA process was the evaluation of alternative methods of implementing the undertaking which involved the identification of a preferred site for the facility.

Prior to the final site-selection and the vendor-selection processes, the EA public consultations raised concerns for emission of contaminants and human health effects (Jacques Whitford Limited, 2009a). Concerns of EfW plants and emissions allowed for the Regions to opt for a generic HHRA as part of the EA study. The assessment was not meant to be site-specific until the actual site was chosen; it was used as a feasibility study to identify potential issues that should be monitored during a site-specific HHRA (Jacques Whitford Limited, 2009a).

After careful considerations of the advantages and disadvantages, the preferred site for the thermal treatment facility was Clarington 01 (Jacques Whitford Limited, 2009a), located in the Region of Durham, south of the 401 in the Municipality of Clarington. A vendor identification process followed the site-selection. Here, a competitive process was undertaken to identify a preferred vendor for the thermal treatment technology and Covanta Energy Corporation was selected. Covanta, the largest provider of thermal treatment services in all of North America with numerous facilities already in the United States, agreed to design, build, and operate the incinerator (Ollson et al., 2014).

The final step was a completion of site and vendor specific studies to assess the undertaking. This was done to assess the potential effects that were associated with the 
thermal treatment plant. The studies that were conducted included an Air Quality Assessment, Natural Environment Impact Assessment, Acoustic Assessment, Traffic Assessment, Visual Assessment, Economic Assessment, Social/Cultural Assessment, Geotechnical investigation, Surface Water and groundwater Assessment, Stage 2 Archaeological Assessment and Built Heritage for the Clarington 01 Site, Facility Energy and Life Cycle Assessment and the SiteSpecific Human Health and Ecological Risk Assessment which is the central focus of this study (Jacques Whitford Limited, 2009a).

\subsection{Description of the Durham-York Facility}

The proposed facility has an initial design capacity of 140,000 tpy with a maximum capacity of 400,000 tpy (Ollson et al., 2014). For the initial design capacity, the facility will consist of two independent waste processing trains with each consisting of a feed chute, stoker, integrated furnace/boiler, dry recirculation acid gas scrubber, a fabric filter bag house and associated ash and residue collection systems (Ollson et al., 2014). Once steam is produced in the boiler, it will drive a turbine-generator to produce electricity to be used for plant-use or district heating (Ollson et al., 2014). The thermal treatment process will take place after the removal of recyclable materials and any other diversion of material as necessary. The ash that is left over post-incineration, will be disposed of in a landfill or could be used for construction purposes to build roads (Ollson et al., 2014). The maximum design capacity would include two additional waste processing trains (Ollson et al., 2014). The human health concern is raised 
because although emission treatment is advanced, there will be fugitive emissions that will affect local exposed populations.

Within the 35 year planning period, there is a potential that the facility may need to be expanded. Therefore, potential phases of expansions have been planned; a phase one expansion of 250,000 tpy and the second expansion of 400,000 tpy, which is the maximum design capacity (Jacques Whitford Limited, 2009a). The EA approval is for the 140,000 tpy and any future expansion will require an amendment to the current EA (Januszkiewicz, 2013).

\subsubsection{Air Pollution Control}

Proponents of the Durham-York EfW facility have stated that it will follow stringent air emission standards to adhere to the Ontario A-7 Guideline: Air Pollution Control, Design and Operation for thermal treatment plants in Ontario as well as Ontario Regulation 419/05: Air Pollution-Local Air Quality. This document was created in order to assist applicants applying for air Certificates of Approval ( $\mathrm{C}$ of $\mathrm{A}$ ) for MSW facilities. A C of A is a document required by the Ontario Environmental Protection Act for projects that are related to air and waste management activities (Ontario Ministry of the Environment, 2010). It is a site-specific document that outlines the responsibilities for the operators of the facility in order to protect human health and the environment from harmful emissions. It requires a facility to conform to generally accepted engineering practices as well as provide minimum compliance requirements for facility operators (Ministry of the Environment, 2010). For air Cs of A, an Emission Summary and Dispersion Modelling (ESDM) Report is required along with the Cs of A application. 
Figure 2.1: Overview of the Durham-York Environmental Assessment Process (Adapted from Jacques Whitford Limited, 2009a)

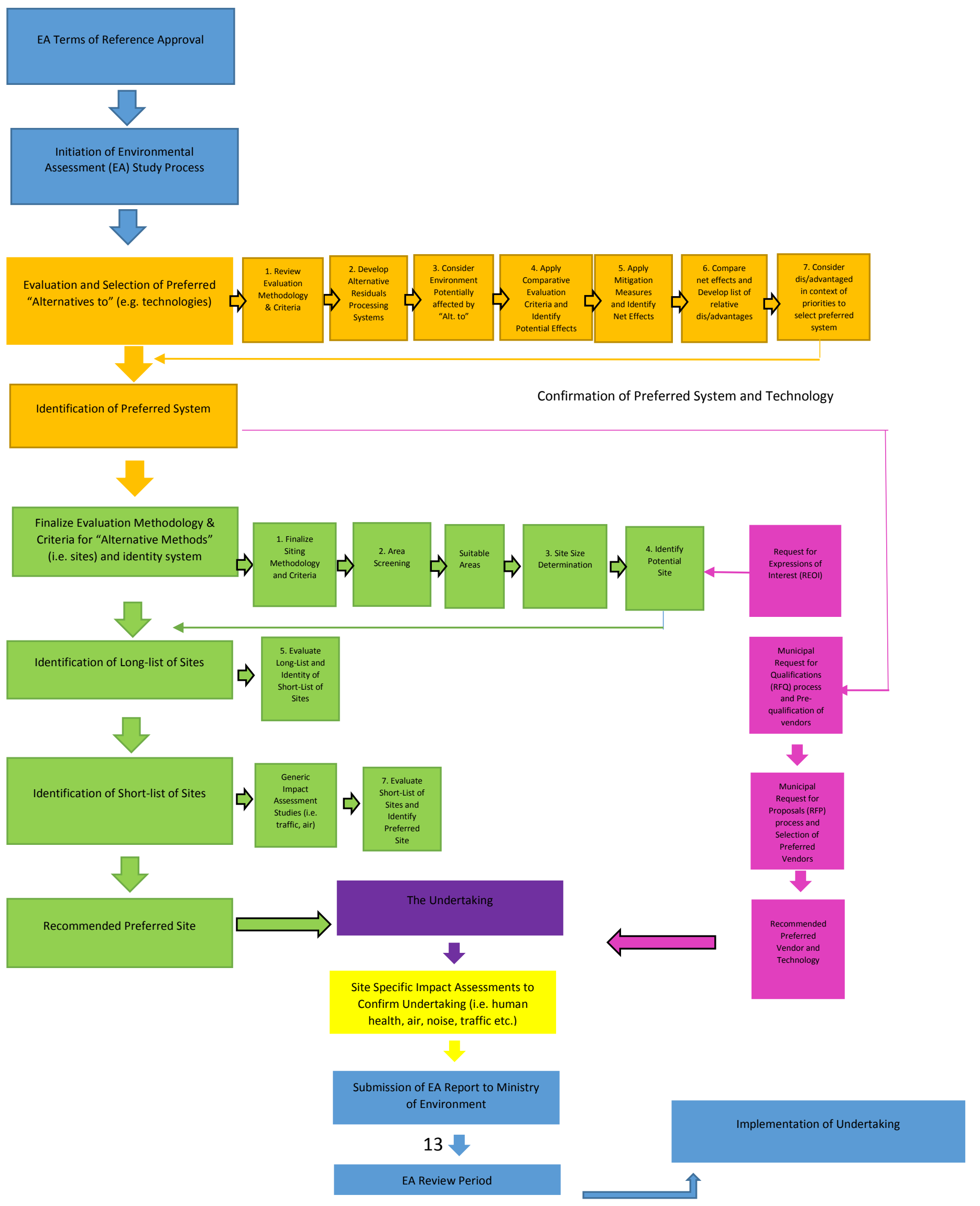


This report is to be prepared in accordance with the Ministry's guidelines and is to contain an assessment of all emissions of common contaminants from the facility (Ministry of the Environment, 2010).

The APC technology consists of a selective non-catalytic reduction system (SNCR System) with ammonia injection to control nitrogen oxides $\left(\mathrm{NO}_{\mathrm{x}}\right)$; an activated carbon injection system to reduce mercury and dioxins and furans in flue gas; a dry recirculation lime injection scrubber to control acid gases; and a pulse jet type highly efficient bag house to control particulate emissions (Jacques Whitford Limited, 2009a).

The $\mathrm{NO}_{\mathrm{x}}$ control system has been designed to work with two systems to control $\mathrm{NO}_{\mathrm{x}}$ emissions. The first is Covanta's VLN ${ }^{T M}$ (Very Low $\mathrm{NO}_{\mathrm{x}}$ ) system which uses a unique combustion air design along with an advanced combustion monitoring design to achieve a considerable reduction in the $\mathrm{NO}_{x}$ emissions (Jacques Whitford Limited, 2009a). The second system that will be used to control the emissions is the SNCR system, which when paired with the VLN ${ }^{\mathrm{TM}}$ system, will help in achieving $40 \%$ less emissions than that outlined in the MOE Ontario A-7 guideline mentioned above (Jacques Whitford Limited, 2009a).

The activated carbon injection system is another APC technology that will be utilized in the Durham-York facility to control mercury and dioxin/furan releases into the environment. Activated carbon particles will be injected into the flue gas to adsorb pollutants on their surface and then these carbon particles will be trapped by a baghouse (Jacques Whitford Limited, 2009a). In addition to carbon particle removal, the fabric filter baghouses will be used to remove fly ash particles, scrubber reaction products and unreacted lime by flue gas (Jacques 
Whitford Limited, 2009a). Acid gas scrubbers will also be used in the facility to reduce acidic gas emissions and will be either a semi-dry design or a circulating dry design (Jacques Whitford Limited, 2009a).

Overall, the APC equipment used throughout the facility is to ensure that there will be no exceedances of air emissions in accordance with Ontario MOE guidelines that are in place to control the release of contaminants into the atmosphere. However, even with modern APC, emissions have the possibility of releasing into the atmosphere and sometimes in excessive amounts, therefore, the HHRA needs to ensure that pregnant women and fetuses and other vulnerable sub-populations are effectively protected from this.

\subsection{Contaminants of Potential Concern and Potential Health Effects}

Despite modern incinerators such as the Durham-York one being equipped with advanced APC technology, they still emit potential harmful toxins, depending on the composition of the waste and some may still potentially be emitted into the atmosphere as discussed later in this section. The contaminants of primary concern include carbon monoxide (CO), carbon dioxide $\left(\mathrm{CO}_{2}\right)$, nitrogen oxides $\left(\mathrm{NO}_{\mathrm{x}}\right)$, sulfur dioxides $(\mathrm{SO})$, hydrogen chloride $(\mathrm{HCl})$, cadmium $(\mathrm{Cd})$, lead $(\mathrm{Pb})$, methane $\left(\mathrm{CH}_{4}\right)$, mercury $(\mathrm{Hg})$, particulate matter $(\mathrm{PM})$, chromium $(\mathrm{Cr})$, arsenic (As), beryllium (Be), dioxins (PCDDs) and furans (PCDFs), polychlorinated biphenyls (PCBs), and polycyclic aromatic hydrocarbons (PAHs) (National Research Council, 2000;

Snary,2002). Several studies have shown that the public has raised concerns about health risks posed by the process of incineration and the contaminants that are produced by such 
facilities (Snary, 2002). Several contaminants listed above have the potential of affecting different organs in humans and in animal bodies and the degree of harm is dependent upon the chemical entity and duration and extent of exposure (Chance, 2001).

\subsubsection{Teratogens}

Teratogens are a category of contaminants that are primarily of concern for the developing fetus. They are defined as environmental factors that can produce permanent abnormalities in structure or function of a fetus or growth restrictions (Frias and GilbertBarness, 2008). The first trimester of pregnancy is associated with increased rates of malformations whereas exposures during the second and third trimester can cause risks of growth restrictions of the exposed fetus (Holmes, 2011). The organogenesis period is one of the most critical stages of exposure for teratogens and can lead to many structural defects as well (Frias and Gilbert-Barnes, 2008). Fetuses are also known to have higher rates of cell proliferation and cell differentiation which provides further reason for their higher levels of sensitivity (Llop, Ballester, Estarlich et al., 2010).

Dioxins, mercury, lead, cadmium, chromium, arsenic, and PCBs are all examples of teratogens. Due to the limitations of research and difficulty of isolating the relationships between the contaminant and their effects, studies are unable to provide $100 \%$ accurate evidence that a contaminant can cause a specific effect. However, several studies have shown that association of MSW contaminants and the prevalence of detrimental outcomes with a high probability that the developmental defects are due to the contaminants. Vinceti, Malagoli, Teggi et al. (2008) studied rates of spontaneous abortion and the prevalence of congenital 
anomalies in women who resided near a MSW facility in Modena, Italy. Although their evidence was non-conclusive, they did find an increased risk of birth defects in female workers who worked in facilities near the exposed area (Vinceti et al., 2008). A study by Candella, Ranzi, Bonvicini et al. (2013) investigated eight incinerators in Italy to determine the relationship between the MSW and pregnancy outcomes. After analyzing women who resided within a 4-km radius from MSW facilities, they found that maternal exposures was associated with pre-term delivery, even at low levels (Candella et al., 2013). A more recent study by Candella, Bonvicini, Ranzi et al. (2015) stated with certainty that exposure to incinerator emissions was associated with an increase of miscarriages in pregnant women. This result came after the analysis of pregnant women residing within 4-km of seven MSW incinerators in Italy (Candella et al., 2015).

\subsubsection{Contaminants of Potential Concern and their Associated Health Effects}

Polychlorinated dibenzodioxins (PCDDs) and polychlorinated dibenzofurans (PCDF) most commonly known as dioxins and furans, respectively, are gaseous products known as persistent organic pollutants (POP) that are of most concern among incinerator emissions and have been subjects of the most controversy due to their toxic profiles. Dioxins-which are a likely human carcinogen produced when wastes are burned-may enter into humans by absorption into the lungs or by deposition onto skin, but according to Snary (2002) and Health Canada (2005a) the major pathway of this contaminant is through the food chain. It has been estimated that $90 \%$ of people's overall exposure to dioxins and furans are to be from their diet (Health Canada, 2005a; World Health Organization, 2014). Meat and milk products as well as fish are known to contain higher levels of dioxin and furans than fruits, grains and vegetables (Health Canada, 
2005a). Of the known dioxins and furans, 2, 3, 7, 8- TCDD has been characterized as the most toxic member of the dioxin family and a known human carcinogen (Snary, 2002; Health Canada, 2005; Agency for Toxic Substances and Disease Registry, n.d).

Long-term exposure of dioxins and furans also have the potential to alter hormone levels in animals and humans (Health Canada, 2005a; World Health Organization, 2014; Agency for Toxic Substances and Disease Registry, n.d); impair the immune system (World Health Organization, 2014; Agency for Toxic Substances and Disease Registry, n.d), and affect the endocrine system as well as reproductive functions (Health Canada, 2005a). Short-term exposure to dioxins and furans can result in skin disorders such as chloracne (Health Canada, 2005a; World Health Organization, 2014; Agency for Toxic Substances and Disease Registry, n.d). Dioxins have also been found to affect fetal development due to their carcinogenic nature (Health Protection Scotland, 2009). Tango, Fujita, Tanihata et al. (2004) conducted a study assessing MSW incinerators in Japan. In a study area within $10 \mathrm{~km}$ from 63 municipal solid waste incinerator, Tango et al. (2004) found that there were 3387 fetal deaths, and 835 infant deaths potentially associated with the exposures of contaminants. Due to the lack of information regarding dioxin exposures around the facilities, it could not be strongly proven that the dioxins were the cause of these deaths or any congenital malformations (Tango et al., 2004). However, this does not mean that dioxins do not have the potential for these types of effects, this study just proves the difficulties of this type of research (Tango et al., 2004).

The event of the Agent Orange exposure during the Vietnam War also highlights the detrimental effects of dioxins. The United States army sprayed millions of gallons of Agent Orange to defoliate and destroy crops and even decades later, the health effects associated 
with the exposure is associated with health effects to the offspring of the veterans (United States Department of Veteran Affairs, 2014). Agent Orange consisted of high levels of the most potent dioxin of the dioxin family, known as 2,3,7,8 TCDD, classified as a harmful carcinogen (Clapp, Baraldi, Grassman et al., 2014). Health effects included defects in the developing fetus causing spina bifida ( United State Department of Veteran Affairs, 2014), cleft lip, cleft palate as well as congenital heart disease ( Clapp et al., 2014). In addition, the exposure was associated with infant mortality, miscarriage, pre-mature birth as well as other types of congenital abnormalities (Palmer, 2007).

Heavy metals, including mercury are also of concern as they are released from MSW incinerators (Thompson and Anthony, 2008). The dangers of mercury were made more prominent with the outbreak of Minimata disease in Japan in 1956. Methylmercury is a toxic compound of mercury which caused numerous deaths and led to chronic damage to the nervous system for many victims (Harada, 1995; Kuntz, Ricco, Hill et al., 2010). This neurotoxin has been linked to learning disabilities and hyperactivity as well as Alzheimer's disease (Thompson and Anthony, 2008). Similarly, the dangers of mercury were apparent with the English-Wabigoon River contamination that occurred during 1962 to 1970, when an alkaline chlorine plant dumped their mercury waste into the river (Kinghorn, Solomon and Chan, 2007). The health effects that were prevalent in Minimata were also seen in humans who were contaminated by the English River and research presumed it to be from high levels of methylmercury contamination (Takaoka, Fujino, Hotta et al., 2013). Methylmercury has devastating effects with pre-natal exposures as well, resulting in impaired child neurological development (Oken and Bellinger, 2008). 
Arsenic, lead, cadmium, beryllium and chromium are also very toxic to humans as they all have potential carcinogenic effects. Arsenic exposure to humans and animals occurs through drinking water, and low levels of arsenic in the body has led to acute effects such as vomiting, skin effects, diarrhea (Health Canada, 2008a) and respiratory irritation (National Research Council, 2000; Agency for Toxic Substances and Disease Registry, 2007). Higher levels have led to cancer of the lungs, bladder, skin, kidney, nasal passages, liver a well as the prostate (Chance, 2001; United States Environmental Protection Agency, 2013a). The Agency for Toxic Substances and Disease Registry (ATSDR) has found evidence that arsenic can harm pregnant women and their fetus (Agency for Toxic Substances and Disease Registry, 2007). Animal studies with arsenic have also shown that pregnant females are more likely to fall ill and exposure can cause fetal malformations or even fetal death (Agency for Toxic Substances and Disease Registry, 2007). In addition, arsenic has the ability to cross the placenta barrier and has been observed in a mother's breast milk at low levels (Agency for Toxic Substances and Disease Registry, 2007). Health Canada (2008a) has also stated that infants and children are found to be more susceptible to the toxic effects of arsenic due to their smaller body sizes and weights.

Chronic exposure to cadmium results in toxic effects, particularly to the lungs and can potentially cause emphysema and proteinuria (Health Canada, 2008b). Chronic exposure can lead to a decrease in liver function as well as liver diseases (Health Canada, 2008b) and kidney damage (Statistics Canada, 2008). It is a known carcinogen as it has been associated with lung cancer (Statistics Canada, 2008).Cadmium is a contaminant known to have adverse reproductive health effects along with lead and mercury (Ashworth et al., 2014). 
Beryllium has been linked to an increased risk in lung cancer (Agency for Toxic Substances and Disease Registry, 2002a; Thompson, 2005); making it a probable human carcinogen (Agency for Toxic Substances and Disease Registry, 2002a). Dermal exposure to beryllium can lead to skin allergies and chronic inhalation of the contaminant has been cited as a cause of immunological effects in humans and animals (United States Environmental Protection Agency, 2000). Huai, Feng-Yuan Xiao-Wei et al. (2010) reported that beryllium has been associated with reductions in fetal and placental weights as well as stillbirths.

Lead is another heavy metal known to be stored in body tissues, blood, the kidney, brain and in bones (National Research Council, 2000). Ongoing research of the contaminant shows that safe levels of lead for children are non-existent and this raises concern (McClenaghan, Cooper, Muldoon et al., 2003). Children are found to absorb about $30 \%$ more lead through the gastrointestinal tract more than adults (National Research Council, 2000). This is dependent on dietary factors such as the types of vitamins and other essential nutrient deficiencies that can affect the amount of lead that is ultimately absorbed (National Research Council, 2000). Furthermore, maternal exposure to lead has been reported to increase the risk of abortions (Borja-Aburto, Hertz-Picciotto, Rojas Lopez et al., 1999), pre-term delivery and low birth weight (Health Protection Agency, 2012). In addition, lead has toxic effects that could potentially affect the developing nervous system of the fetus (Chance, 2001).

The health effects of chromium depend on its different speciation forms. The most common forms of chromium are chromium (0), chromium (III) and chromium (VI) (Agency for Toxic Substances and Disease Registry, 2008). Hexavalent chromium (chromium (VI)) is more toxic than chromium (III) and it is known to be carcinogenic to humans. It can cause lung cancer 
(Health Canada, 2010) as well as gastrointestinal and neurological effects (United States Environmental Protection Agency, 2013b).

$\mathrm{SO}_{\mathrm{x}}, \mathrm{NO}_{\mathrm{x}}, \mathrm{PM}, \mathrm{CO}$, and $\mathrm{NH}_{3}$ (ammonia) are common air pollutants (Environment Canada, 2013a) that have the potential to cause human and environmental health effects. Specifically, $\mathrm{SO}_{2}$ has short-term and long-term health effects associated with its exposure. The short-term effects of inhaling this contaminant include respiratory effects such as bronchoconstriction and other respiratory illnesses while long-term effects include respiratory diseases such as emphysema (United States Environmental Protection Agency, 2014a). $\mathrm{NO}_{2}$ health effects include respiratory effects such as air inflammation and when combined with other contaminants such as ammonia, can cause or worsen respiratory diseases (United States Environmental Protection Agency, 2014b). A study by Shah, Balkhair et al. (2011) found that exposures to SOx was associated with pre-term delivery and a study by Llop et al. (2011) further confirmed that SOx and NOx were associated with pre-term birth.

Due to the nature of PM, the ultra-fine particles are responsible for the various health effects since they can get deep within the lungs and blood stream (United States Environmental Protection Agency, 2014c). Effects include premature death for those with heart or lung disease, decreased lung function, increased respiratory symptoms as well as non-fatal heart attacks (United States Environmental Protection Agency, 2014c). PM is particularly dangerous as it allows for the strong tendency of adsorption of contaminants such as dioxin (McKay, 2002), arsenic (Hu, Liu, Chen et al., 2015) which eventually are what make contact with receptors. A study by Dejmek, Selevan, Benes et al. (1999) found that exposure to PM during the first gestational month was a critical period for fetuses by adversely affecting fetal growth. 
In addition, the study revealed the possible risk of intrauterine growth retardation resulting from exposure to PM (Dejmek et al., 1999). A recent study by Zhu, Liu, Chen et al. (2015) investigated the relationship between maternal exposure to PM2.5 during pregnancy and found that it can increase the risk of low birth weights and pre-term births.

$\mathrm{CO}$ is formed during the combustion process as a result of carbon not being completely oxidized and can reduce the oxygen-carrying capacity of blood due to its high affinity to hemoglobin compared to oxygen (National Research Council, 2000; United States Environmental Protection Agency, 2014d). Due to the lack of blood getting to the body's organs, high levels of CO can result in death in adults and the fetus (United States Environmental Protection Agency, 2014d). Ammonia is a gas that is poisonous if inhaled in large quantities, causing lung damage (Agency for Toxic Substances and Disease Registry, 2004; Environment Canada, 2013b). In lesser amounts it can irritate the eyes, nose and throat (Environment Canada, 2013b).

Other common air contaminants include $\mathrm{HF}$ and $\mathrm{HCl} . \mathrm{HCl}$ is an irritant that can cause respiratory problems (Agency for Toxic Substances and Disease Registry, 2002b). HF is a very strong acid that is irritant to the eyes, nose, and respiratory tract (Agency for Toxic Substances and Disease Registry, 2003). Large amounts of HF on the skin can cause death by the fluoride damaging the heart or lung (Agency for Toxic Substances and Disease Registry, 2003).

PAHs are produced during incomplete combustion and the routes of exposure include ingestion of contaminated water, as well as inhalation of contaminated air (United States Environmental Protection Agency, 2008a), dermal contact with air, water, or soil near the waste 
site, or even through a mother's breast milk (Agency for Toxic Substances and Disease Registry, 1995). Through laboratory studies, it has been determined that some PAHs are known to cause cancer (Agency for Toxic Substances and Disease Registry, 1995). High levels of PAHs have also been associated with developmental defects for pregnant mice (Agency for Toxic Substances and Disease Registry, 1995; United States Environmental Protection Agency, 2008a). The target organs of PAH are usually the kidney and liver (United States Environmental Protection Agency, 2008a).

PCBs are persistent mixtures (Agency for Toxic Substances and Disease Registry, 2000) and their major exposure routes are through ingestion of contaminated foods (such as fish, game and marine mammals) and contaminated water, inhaling polluted air and having dermal contact (Agency for Toxic Substances and Disease Registry, 2000). In humans, PCBs have been associated with liver cancer (Agency for Toxic Substances and Disease Registry, 2000; Health Canada, 2005b), kidney cancer (Health Canada, 2005b) as well as cancer of the biliary tract (Agency for Toxic Substance and Disease Registry, 2000). In addition, women who were exposed to PCBs or consumed large amounts of contaminated fish gave birth to babies who had lower birth weights, problems with motor skills and responses, a decrease in short-term memory as well as an affected immune system compared to women who were not exposed to such levels of PCBs (Agency for Toxic Substances and Disease Registry, 2000).

Aside from gaseous effluents, the incineration process also produces byproducts such as soot and incombustible waste known as bottom ash, which must ultimately be disposed of in a landfill (National Research Council, 2000). Soot and bottom ash also have health implications but due to the scope of this research, they will not be discussed. 
Due to the number of different types of contaminants and their properties, only a few have been discussed here. However, it is important to note that developing fetuses are highly vulnerable to toxins, particularly to carcinogens (Huai et al., 2010). Fetuses undergo rapid cell differentiation and cell proliferation and carcinogens can easily impede this process (Huai et al., 2010). Although literature suggests that there are concerns about human health risks arising from incinerators, there are no conclusive studies which can support that incinerators can directly cause health effects (Snary, 2002). This is due to the fact that the incidences of health effects cannot be exclusively linked to the emissions from incinerators due to the background exposures of contaminants that exist. This makes it difficult to assess the direct relationship of emissions and adverse effects. Consequently, best available techniques and practices should be utilized when assessing the health risks of emissions to protect human health as much as possible.

This discussion of these contaminants provides insight into the concerns of the contaminants released from incinerators. Specifically, it draws attention to fetal effects associated with the exposure to these harmful contaminants, identifying that the inclusion of pregnant women and fetuses should be a best practice when conducting human health risk assessments.

\subsubsection{Emission Exceedances for Facilities with Modern Air Pollution Control Technologies}

In recent years, increasingly stringent limits have been imposed on atmospheric emissions from municipal solid waste incinerators (Sabbas, Speiser, Heiss-Assbichler et al., 2001), and modern APC technology have controlled the amounts of pollutants released into the 
atmosphere. Despite, modern air control technologies such as scrubbers and filters, there are still emissions since the air control pollution technologies, although advanced and effective, do not eliminate all pollutants (Takahashi et al., 2010).

Even modern day incinerators have had several occurrences of emission exceedances deviating from regulatory limits. In recent years, Covanta has had exceedances of permitted contaminant levels from their incineration facilities in the US. In 2008, Covanta's facility in Wallingford, Connecticut produced levels of dioxins and furans $250 \%$ above the allowable permit and regulatory limit (Department of Energy and Environmental Protection, 2011). From 2004 to 2008, Covanta's facility in Lancaster, Pennsylvania had increased levels of carbon monoxide, sulfur dioxide, hydrogen chloride and nitrogen oxide exceeding the regulatory limits (Commonwealth Pennsylvania Department of Environmental Protection, 2009). Another exceedance of dioxins and furans occurred at Covanta's Pittsfield, Massachusetts facility when its dioxin and furan levels surpassed the regulatory limits by $350 \%$ (Commonwealth of Massachusetts Department of Environmental Protection, 2008). This raises concerns about the public health and safety with Covanta operating the Durham-York facility and raises the question of whether vulnerable populations remain at risk despite advanced APC technology.

\subsection{Susceptible Populations of Focus: Pregnant Women and Fetuses}

HHRAs should take into account vulnerable population segments to accurately determine their health risks. While certain segments such as children and the elderly are extensively studied in the literature, effects on pregnant women and fetuses have not been 
widely reported in current HHRA literature. Therefore, they were chosen as the primary focus of this study. This study will contribute to the limited niche of research on pregnant women and fetuses and their consideration in HHRAs.

Health Canada (2011) states that the fetus has the possibility of being exposed to harmful contaminants via the placenta. According to Landrigan, Kimmel, Correa et al., (2004) fetuses are uniquely vulnerable to environmental toxicants, and are up to 10 times more vulnerable to some contaminants (National Academy of Sciences, 1993) therefore, they require special considerations in risk assessments. A primary reason for their vulnerability is due to the critical windows of development, which explains their unique patterns of exposure to environmental toxicants (Landrigan et al., 2004). Critical windows of development are defined as a time interval when a subpopulation-in this case pregnant women and fetuses-are more sensitive to a chemical exposure than at other time periods (Selevan et al., 2000). According to Chance (2001), human growth is the most rapid in utero and in early childhood stages, as well as puberty, and therefore, are the time periods where the contaminants can damage the body the most. Specifically for fetuses, the most critical time period is during the first trimester of development, during the organogenesis phase which leads to rapid development of tissues and organs and continues to develop in infants and young children (United States Food and Drug Administration, 2005; Diav-Citrin, 2011; Felter, Daston, Euling et al., 2015). Therefore, exposure during this time could lead to extensive damage to multiple organ systems.

The fetal life-stage refers to a particular stage of development. The term life-stage refers specifically to a period of life that may have distinct characteristics which contribute to the potential differences in vulnerability to different contaminants (United States Environmental 
Protection Agency, 2006; Brown, Barone, and Kimmel, 2008). The distinction of life-stages is important when accounting for health effects because exposures during different fetal lifestages result in different adverse effects. Exposure pathways for this life-stage differ from other receptor groups as well; for example, the fetus would receive exposures trans-placentally from the mother. With pregnant women exchanging about $72 \%$ more air over an 8 hour period at rest than non-pregnant women as Selevan et al. (2000) found, concerns increase with the likelihood of inhaling environmental toxicants at a greater amount which could potentially be transferred from the mother to the fetus.

It is important to note that most of the chemicals that are harmful to humans are fat soluble, therefore the chemicals are stored in the fatty tissues of the nervous system (Dejmek et al., 1999). Mercury is a good example, because prenatal exposure to methylmercury affects neurodevelopment that results in the trans-placental transfer of mercury from maternal blood to the fetal brain (Landrigan et al., 2004) and this can cause neurodevelopmental damage. In addition, the fetal blood-brain barrier is not fully developed, therefore substances are readily available to cross into the central nervous system (Rodier, 1995). The World Health Organization (WHO) has also drawn an association between dioxins and developing fetus, stating that it is the most sensitive to dioxins compared to other vulnerable populations, creating a greater health impact on them (World Health Organization, 2014).

Vulnerability of the fetus and pregnant women was illustrated by a study conducted by Farlie (2007) and provided the inspiration for this study. In his study on Canada's nuclear power plants, looking specifically at the contaminant tritium, he explicitly stated that based on local exposures, pregnant women should not live within $10 \mathrm{~km}$ of a nuclear power plant 
(Farlie,2007). Although tritium and nuclear plants are not a focus of this research, nor are the mechanisms of the contaminants the same, Farlie's (2007) finding provides credence to this sub-population being uniquely vulnerable and affected by the release of environmental contaminants more than the general population. In addition, it was interesting that Farlie gave such a stark warning in regards to the sensitivity of this sub-population. For this reason, it was mentioned in this study.

\subsection{Human Health Risk Assessments}

HHRAs are structured, systematic, decision-making tools (National Research Council, 1996; Calow, 1998) that are used to determine the fate and transport of chemicals in the environment including health risks associated with the inhalation, ingestion and dermal contact with contaminants (Snary, 2002; Government of Alberta, 2011). In the context of incineration, the health risks associated with the emissions are what will be analyzed in the HHRA. A formal definition of an HHRA is provided below. A HHRA is:

A process used to estimate the nature and probability of adverse health effects in humans who may be exposed to chemicals in contaminated environmental media, now or in the future (Ontario Ministry of the Environment, 2005; United States Environmental Protection Agency,2012a).

Not only have risk assessments been used to describe the probability of an adverse event from chemical and physical agents, they have also been used to describe a series of 
unwanted events such as industrial explosions and a wide variety of natural disasters (Paustenbach, 1989). The most basic form of risk assessment can be traced back to $3200 \mathrm{BC}$ (Covello and Mumpower, 1985) (Cited in Schulte [2003]) but modern risk assessment is much more complex.

Risk assessments became increasingly popular after military operations research in the 1950's to avoid chemical and nuclear plant failure (Demidova and Cherp, 2005), but prevailed after the 1976 dioxin release in Seveso, Italy as well as the 1984 methyl-isocyanate incident in Bhopal, India (World Bank, 1997). Industrial pollutants have been an increasing human health concern and have gained widespread attention, particularly after the release of Rachel Carsons' Silent Spring in 1962 (National Resources Defense Council, 2013). Risk assessments assist policy makers and regulators to make informed decisions about the health risks that have the potential to harm humans (Paustenbach, 1989).

There are five different components for a complete HHRA which include the problem formulation/screening stage, the hazard identification stage, the exposure assessment stage, the dose-response/toxicity assessment stage and finally the risk characterization stage. Problem formulation/Screening is the first stage of the risk assessment process that involves the screening of chemicals and identification of chemicals of potential concern, identification and description of potential receptors (including sensitive receptors) as well as identification of exposure pathways associated with the project (Government of Alberta, 2011; Health Canada, 2012). The main product of this stage is the development of a conceptual site model that describes the project and the interactions the chemicals have with receptors via different exposure routes and to determine the chemicals, pathways and risk that have the greatest 
potential of creating a health risk (Government of Alberta, 2011). This stage provides the scope of the HHRA.

The second stage of the HHRA is the hazard identification stage. In many risk assessments, hazard identification is used in place of the problem formulation stage, or vice versa, but for the purpose of this study, both stages will be used, since both are quite distinct in description and nature. Problem formulation focuses on the screening and scoping of the HHRA while hazard identification focuses its process on whether exposure to a specific contaminant can cause an increase incidence of specific adverse health effects and whether these health effects are likely to be exhibited in humans (United States Environmental Protection Agency, 2012). Specifically, this stage determines if chemicals of concern have the potential to increase the incidence of cancer in humans (National Academy of Sciences, 1983) and is observed via animal and human-based toxicity studies.

The next stage is the exposure assessment stage. It involves identifying the population(s) which might be exposed to the agent of concern, the specific routes in which exposure may occur as well as estimating the magnitude, frequency and duration of the potential exposure of the contaminants of concern (National Research Council, 1996; Snary, 2002; Ministry of the Environment, 2005).

Next is the dose-response stage which focuses on the relationship between the magnitudes of exposure and the probability of an adverse health effect as a result (Ministry of the Environment, 2005). Here, toxicity reference values (TRVs)-acceptable doses or concentrations of a contaminant of concern that can be tolerated by a receptor without causing 
an adverse health effect-are identified (Health Canada, 2010). The TRVs should consider vulnerable populations when formulating tolerable concentrations. This stage is key for the protection of vulnerable populations in HHRA.

Risk characterization is the final stage which integrates the previous stages of the risk assessment to determine the probability that an emission from the facility causes harm to the receptors of concern (National Research Council, 1996; Snary, 2002). In other words, this stage makes risk estimations using quantitative or qualitative analysis (Ministry of the Environment, 2005) and also discusses the uncertainties that are present in the risk assessment as a whole (National Research Council, 1996; Snary, 2002).

These stages are important in formulating a health risk assessment, but it is crucial that the HHRA is centered on protecting sensitive populations, not just the general population. Pregnant women and fetuses are usually not the focus in a HHRA, therefore it becomes questionable whether they are truly being protected from facilities such as incinerators.

\subsubsection{Human Health Risk Assessments for Pregnant Women and Fetuses}

Historically, risk assessments have focused on adult exposures and toxicities giving little consideration to the fetus and fetal development (Landrigan et al., 2004). Default values in the past were based on a healthy young adult and did not account for the sensitivities and unique characteristics of the fetus (Landrigan and Carlson, 1995). The risk assessment for this population needs to move beyond consideration of average levels of exposure and risk and the 60-70 kg average adult (Landrigan et al., 2004). Although risk assessments have started 
considering "worst-case" scenarios in their calculations, the consideration of unique susceptibilities are not taken into account and are often generalized.

As the WHO (2008) stated, assumptions cannot be made about children assuming they are little adults, which implies the need for their unique consideration in HHRAs (Olin and Sonawane, 2003). For this reason, WHO (2008) proposes a different risk assessment approach to ensure that these vulnerable populations are protected. Daston, Faustman, Ginsberg et al. (2004) agreed by stating that taking embryonic and fetal development into consideration in a risk assessment is as important as life after birth, because these developmental stages affect how a toxicant will behave, either during this developmental period or the effects that may persist after birth. Even using the term "children" or "child" is very general. Cohen Hubal, de Wet, Dur Toit et al. (2014) stated that it was important to consider childhood as sequences of life-stages, ranging from conception to fetal development moving into infancy and adolescent, instead of characterizing children in one subgroup and analyzing them as the same. Due to the changes in anatomy, physiology, metabolism, and behavior, these life-stages are considered different from each other and have the potential of creating higher exposures compared to one another and most definitely compared to adults (Cohen Hubal et al., 2014). From preconception to adolescence, it is important to take into consideration susceptibilities at each development stage particularly because the distinct life-stages are characterized by dynamic processes occurring at molecular, cellular, organ system and organism level (World Health Organization, 2006). As a result, differences in life-stages will determine the severity of the exposures (World Health Organization, 2006). 
The International Life Sciences Institute (ILSI) conference held in 2001 set out to develop a health risk assessment framework for children from exposure to environmental agents (Olin and Sonawane, 2003). The conference established that HHRAs for this sub-population need to recognize the potential significance of "exposure timings in relation to the susceptibility of a developing human and a "systematic approach to the consideration of factors that may influence risk during development from conception to organ maturation" (Olin and Sonawane, 2003:1525). Daston et al. (2004) stated that a risk assessment approach that takes this population segment into consideration needs to acknowledge that the effects of exposures earlier on in life may have a late onset of harmful health impacts. McClenaghan et al. (2003) added that cancer was one of the delayed health consequences to early exposures. Furthermore, risk assessments needs to consider the critical time periods of concern, as this will highlight which organs are targeted and how they are affected (Daston et al., 2004). Therefore, considering a life-stage approach in the HHRA process benefits pregnant women and children and helps create comprehensive analysis of different vulnerabilities that they face (Brown et al., 2008).

A HHRA that protects pregnant women and fetuses differs from the general risk assessment by accounting for various factors that are specific to susceptible populations. This study will examine whether these unique characteristics considered in HHRAs for vulnerable populations were considered in the Durham-York HHRA. Best practices of a HHRA specific to this population will be outlined in detail in the framework, in accordance with experts in the field as well as the literature. 
Prominent sources that consider a life-stage approach and emphasize a focus on vulnerable populations in HHRAs are Selevan et al. (2000); Olin and Sonawane, (2003); Daston et al., 2004; Ginsberg, Slikker, Bruckner et al. (2004); World Health Organization (2006); Brown et al. (2008); Cohen Hubal, Moya and Selevan (2008); Makris, Thompson, Euling et al. (2008); and Cohen Hubal et al. (2014). It is important to recognize that regulatory authorities also agree with the life-stage approach to risk assessments. This includes the USEPA with their risk assessment guidance entitled A Framework for Assessing Health Risks of Environmental Exposures to Children (United States Environmental Protection Agency, 2006) as well as the WHO with their document titled Principles for Evaluating Health Risks in Children Associated with Exposures to Chemicals (World Health Organization, 2006). However, these risk assessment guidance documents are not being used widely in facility risk assessments. Ontario currently does not have a document outlining a life-stage risk assessment process.

\subsubsection{Inaccuracies of Human Health Risk Assessments}

There have been a few comprehensive studies conducted with findings that portrayed the inadequacies of HHRAs specifically for incineration projects and for other polluting facilities as well. Two prominent publications by Snary (2002) and Demidova and Cherp (2005) both focused on inaccuracies directly related to incineration projects and therefore are important to mention.

Snary (2002) conducted his study of HHRAs on United Kingdom (UK) incinerators, as incineration in the UK has become quite prominent. For the scope of his study, Snary (2002) chose to focus on the failings present in the exposure assessment and the risk characterization 
sections of each HHRA. In the 19 HHRAs that were reviewed, Snary (2002) found that, in the exposure assessment, 11 out of the 19 risk assessments failed to provide a reasons why an exposure route was selected or not selected. This presents a challenge because HHRAs are required to provide a justification for actions done (Batterman, 2004). In addition, 8 of the 19 HRAs selected the exposure route of inhalation alone and did not consider indirect pathways of exposure (Snary, 2002).

There were significant failings identified in the risk characterization portion of many HHRAs as well (Snary, 2002). For one, Snary (2002) found that all of the 19 HHRAs failed to acknowledge that different risks are calculated with different models which vary in regard to their assumptions and uncertainties. He felt that comparisons of risks that were made in this stage were unrelated (Snary, 2002). For example, comparing the risks of getting cancer to an annual risk of dying in a road accident is not a good comparison by the risk assessor because both are based on different types of modeling (Snary, 2002).

Lastly, Snary (2002) examined whether uncertainty was adequately accounted for in risk assessments. Discussing uncertainties in the risk characterization stage is a necessity as it allows for the refinement of the risk assessment process and it is a requirement of risk assessors to present a clear explanation of the strengths and limitations of their findings (Snary, 2002). Only 6/19 HHRAs identified that risk estimations were associated with uncertainties and 1/6 went into more complex detail about uncertainty (Snary, 2002). Snary (2002) concluded that a comprehensive and regulatory guideline consisting of best practices for incinerator HHRAs would benefit the process of assessing risks that are posed by incinerators because this 
would serve as a useful guide for developers, consultants and regulatory bodies in determining the adequacy of an HHRA (Snary, 2002).

Demidova and Cherp (2005) proposed a model for integration of Environmental Impact Assessment (EIA) and risk assessment based on a best practices approach. In their paper, they evaluated seven Environmental Impact Statements (EIS) for incinerators located in the UK where a HHRA was an element of the EIS (Demidova and Cherp, 2005). An EIA is "a process of systematic analysis and evaluation of environmental impacts of planned activities and using the results of this analysis in planning, authorizing and implementation of these activities (Demidova and Cherp, 2005:412). The authors found that the treatment of human health in EIAs is very poor, therefore introducing a risk assessment into the EIA process would be beneficial as it would strengthen the assessment of health effects in EIAs (Demidova and Cherp, 2005).

General practice of EIA in the UK includes documenting the findings of an EIA in an EIS (Demidova and Cherp, 2005) which includes an assessment of health risks. The study reviewed EISs of modern EfW incinerators, medical waste incinerators and sewage sludge incinerators (Demidova and Cherp, 2005). The analysis of these health assessments found deficiencies in the hazard identification stage because adequate descriptions of the hazardous by-products were not provided (Demidova and Cherp, 2005). In addition, they found that 4 of 7 of the EISs did not provide records of public involvement in the "discussion of possible health impacts of the proposed facility" (Demidova and Cherp, 2005:423). Furthermore, there was evidence that potentially affected receptors were not identified adequately, nor were there baseline contamination of exposure media characterized (Demidova and Cherp, 2005). This poses a 
problem because the baseline provides a starting level of contamination in a particular medium prior to additional exposures from the proposed facilities giving an idea of how contaminated a medium is likely to be. Last but not least, the study found that uncertainties were only discussed in 2 out of the 7 EISs reviewed; and therefore they did not fulfill an important criterion of a HHRA (Demidova and Cherp, 2005). The study concluded by stating that integration of a risk assessment in an EIA would improve the study of health risks posed to humans (Demidova and Cherp, 2005) therefore implying that health risks to humans are currently not assessed adequately for incineration projects.

This section has shown that health risks to humans posed by incinerators have not been appropriately assessed in the past, whether in an independent HHRA or one that was conducted with another type of assessment, as presented in the Demidova and Cherp (2005) paper. The two papers also portray that in fact, best practices have not been implemented fully. Best practices should be used to minimize health risks and ensure that human health is being sufficiently protected. In Ontario, the MOE states that wherever best practices are not used, an explanation as to why they were not should be given (Ministry of the Environment, 2005) which provides a justification of why this study is being conducted. Another reason of why it is needed is because it will limit significant deficiencies and allow to focus on sub-populations such as pregnant women and fetuses, therefore, fast-tracking the HHRA process.

\subsection{Summary}

This chapter began by presenting a general overview of EfW facilities to provide further background. The chapter then reviewed the Durham-York EA process and described the design 
of the facility including its APC technology. After details on the facility, COPCs that are produced and emitted by incinerators were discussed in addition to occurrences where Covanta had exceedances at their incinerator facilities. This was to show that modern APC technology still have the potential to exceed levels of contaminants being emitted supporting why the adequacy of HHRAs is crucial. With the aforementioned background established, the chapter discussed the susceptibility of pregnant women and fetuses and the essential need for their consideration in HHRAs with supporting literature. Two prominent studies that found deficiencies in HHRAs were also observed. The literature review outlined in this chapter builds the foundation and provides a rationale of why this study was conducted. The following chapter will detail the methods used to achieve the objectives of this study. 


\section{CHAPTER 3: METHODS}

\subsection{Introduction}

The purpose of this study is to examine the Durham-York Incinerators' HHRA to ensure that it followed best practices and intended to protect pregnant women and fetuses. This chapter will first outline detailed methods used to complete the research objectives. This included performing a critical review of literature and existing HHRAs and then performing a synthesis of the literature. This was followed by the interview of experts to ultimately create a best practice framework. The criteria chosen for the best practice framework to protect pregnant women and fetuses, are identified a well.

\subsection{Research Methods}

The following research methods were accomplished throughout the study in order to determine whether the Durham-York HHRA conformed to best practices particularly taking pregnant women and fetuses into consideration.

1. First, a general review of HHRAs was conducted based on the literature and on existing HHRAs. The HHRAs chosen were proposed incinerators using modern APC technologies as well as polluting facilities such as coal or combustion plants. HHRAs of other facilities were utilized due to the limited HHRAs available specifically for incinerators. A total of 11 HHRAs $^{(2,12,15,22,24,25,29,32,59,61,63)}$ predominantly from the US due to availability, were analyzed. When best practices were observed, either in the literature or HHRAs, they were noted in an organized excel spreadsheet which then allowed for an easy 
comparison to other literature/HHRAs to determine the number of times the practice was cited. This step was called synthesis of the literature/HHRAs. Snary (2002) used a similar method to capture best practices.

2. Information from these sources was then used as the foundation for an interview template. The interview template consisted of a number of open-ended questions that were concerned specifically with HHRAs for pregnant women and fetuses. The purpose of the interview process was to complement the concepts identified in the literature and to contribute to a deeper understanding of best practices for incinerator HHRAs. The interviews were conducted with leading HHRA practitioners and public health/environmental specialists. Details of the participants' area of expertise is presented in section 3.2.2. Due to ethics, the participants' identity remained confidential.

3. This interview method of capturing best practices was used in a previous study conducted by Mueller (2010), but this method was applied to EAs and used to determine best practices for Health Impact Assessments (HIAs) in Ontario's Nuclear Industry. This method proved to be successful in his paper, therefore it was selected for this research. It allowed to capture data from several different sources and also allowed the structuring of specific questions that ultimately assisted in attaining the research objectives, leaving irrelevant data out of the process.

4. From the literature review, HHRAs and interview process, the best practice framework was created. Some general practices were included in the framework if they were specific for incineration and others were omitted if they were general for all types of 
HHRAs. The experts were given the opportunity to look over the best practice framework in order to confirm the credibility of the research and to observe if what they claimed were best practices were captured by the draft list. Due to busy work schedules, they were unable to comment.

5. The framework was then compared to the Durham-York HHRA to identify the conformities and deficiencies of the document. The framework was set up as a checklist, with a simple "yes" or "no" determining whether the practice was observed and to what extent. From this conclusion was made deciding whether the Durham-York HHRA was conducted according to best practices and adequately considered pregnant women and fetuses. The research method flow diagram is presented below in Figure 3.1 to highlight the different steps taken.

The United States Environmental Protection Agency (USEPA) (1989) stated that a standardized checklist is an essential review tool. This provides justification for using a checklist as a best practice framework to examine whether the Durham-York facility conducted a HHRA according to best practices. The consultation with experts was used to capture data to determine best practices that should be addressed in the framework. Expert input focuses on "gathering data, information and input on issues" that will help in developing the project (Patterson, Meek, Strawson et al., 2007:1612). This is commonly done in the form of written responses as it identifies relevant data that may be missed by the researcher (Patterson et al., 2007). Patterson et al. (2007) also mentioned that expert input can be more formal and structured. This particular method was chosen in the study as it helped the researcher obtain specific research required for analysis. 


\subsection{Qualitative Research}

\subsubsection{Interview Template}

The interview process is a powerful tool when conducting research and has been proven valuable numerous times. The interview template in this study was created after the literature review was conducted. According to Qu and Dumay (2011), it is crucial to collect enough information and gain knowledge on the topic of interest before preparing informed questions to participants. The literature helped identify best practices that drew attention to vulnerable populations, specifically pregnant women and fetuses. Originally, questions were designed and then pooled until they were narrowed down, focusing on ones that were significant for the framework. As a result, a list of 20 open-ended questions were prepared to capture best practices for incineration processes to protect pregnant women and fetuses. Open-ended questions were used as they maintained focus on the research question but did not presume an answer from participants (Seidman, 2006). Furthermore, they allowed participants to provide their own thoughts on things without feeling the need to filter out or limit their responses as a result of how the question was framed (Rubin and Rubin, 2004) Open-ended questions were also used by Mueller (2010) which proved to be successful In his study. After the interview template was created (Appendix C) and was approved by the Ryerson Ethics Committee, the process of recruiting participants was initiated.

\subsubsection{Recruiting Process}

Following the ethics approval of the interview template and other research methods, the recruitment of participants began. Potential participants included: public health specialists, 


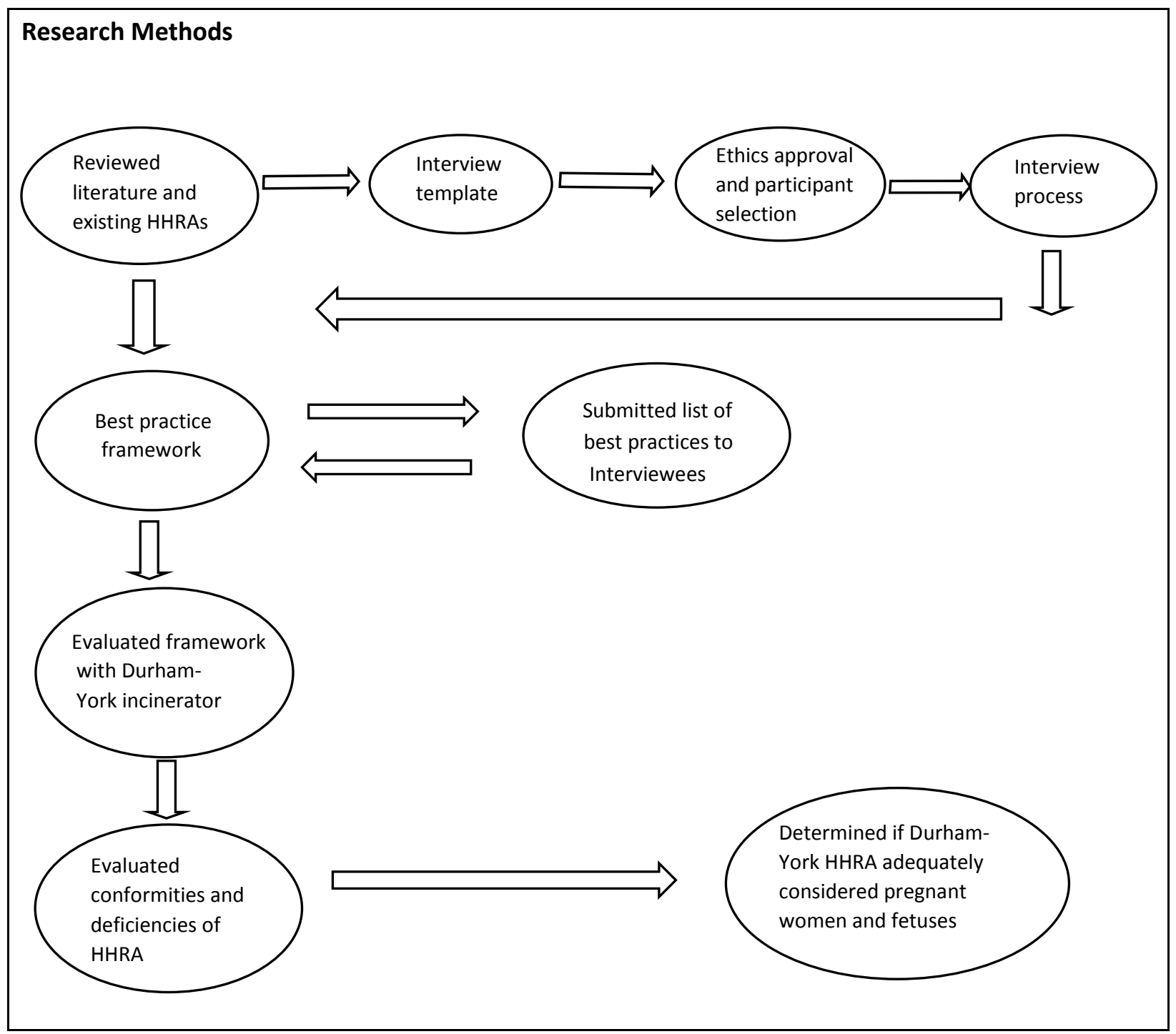

Figure 3.1: Methods used to achieve research objectives

risk assessment specialists/consultants, environmental health/science specialists, toxicologists, and epidemiologists in the environmental and public health fields. These experts were from the government sector, universities or private sectors.

The background of potential participants were researched in detail to confirm that they were able to contribute valuable expertise to the interview questions and that there were no conflicts of interest with people who were apart of the Durham-York project. Unfortunately, 
this project was extensive and many individuals were a part of the approvals process.

Therefore several conflicts of interest arose and resulted in a lower number of participants.

The search process began by contacting authors who had published research on the following: topics of HHRA, general risk assessments, vulnerable populations and HHRAs as well as incineration and public health. Government officials in the areas of public health and HHRAs and Environmental HHRAs were also contacted. Finally, experts such as professors in universities were contacted to participate. The Principal Investigator and the supervisor of this study worked closely to select appropriate participants for the interview process. The individuals contacted were from across Canada, the United States and even the UK. Experts were contacted outside of Canada to ensure a reasonable recruitment number. Since MSW incinerator' HHRAs are not common in Canada as much as they are in the United States and the UK, broadening the scope would allow a greater number of experts in the field to contribute to the study. In addition, it is easier to locate HHRAs for other polluting facilities when the study scope is broadened to consider places outside of Canada.

A recruitment letter/letter of invitation (Appendix B) was prepared according to ethics with information that pertained to the research process, including the purpose of the thesis and the role the participants would play. The consent form (Appendix A) and interview template (Appendix C) were attached to the email for potential participants to review prior to agreeing or declining to partake in the project. The consent form introduced the Principal Investigator, the purpose of the research, how to agree/disagree to be part of the study, next-steps after consent was provided, and any potential risks or discomforts associated with the study. In 
addition, all potential participants were guaranteed full confidentiality in the project meaning their identity would not be disclosed anywhere in the research process.

If the individual did not want to participate, a response to the email was not required, but some responded stating they did not want to or were unable to participate due to conflicts of interest. Following this, no further questions were asked by the Principal Investigator. If an individual agreed to participate, they were required to read and sign the consent form and indicate the method of interview they preferred. For simplicity and ease, the participants could email their consent and their preferred method of interview, stating that they have read the information and agreed to participate in the study.

Potential participants were able to choose from two methods of interview: the telephone interview or the email interview. The email interviews provided flexibility to participants allowing them to respond at their convenience. This method is also beneficial for potential participants who are a long distance away (Ratislavova and Ratislav, 2014). In addition, email interviews allow participants to be more focused, therefore formulate their answers with more detail, and easily review their answers if necessary (Ratislavova and Ratislav, 2014). Written responses are also easier to analyze compared to telephone or face-to-face interviews.

If the participants wished to use the telephone method, they were contacted via email to schedule a time for the interview. Proponents of the telephone method were given the option of being recorded to allow the Principal Investigator to take notes, which all telephone proponents agreed to. Literature has found that this method used in qualitative research is 
successful without issues of data quality (Sturges and Hanrahan, 2004). In addition, the telephone method was easier for those who preferred to talk in person but due to distance (Sturges and Hanrahan, 2004) or their work-load, were unable to do so. The face-to-face method of interview was not given as an option due to time constraints on the Principal Investigators' part, but if the participant requested it, arrangements were made as it is one of the most conventional methods of interviewing participants to receive rich, in-depth data (Lechuga, 2012).

Once an initial letter of invitation was sent to experts, a reminder email was sent a week after to remind potential participants to respond if they wanted to partake in the study. This reminder email proved successful as some of the individuals who wanted to participate but forgot to respond initially, had a chance to do so. Those who did not respond were assumed to have declined to participate and they were not contacted again. For those who had questions before agreeing to participate, their concerns were addressed privately via email. Contact information on those who rejected and who accepted the research consent was organized in an excel spreadsheet by the Principal Investigator. The Principal Investigator was the only individual who has access to this data in a locked spreadsheet, to keep the participants and those who were contacted, anonymous.

\subsubsection{Participation Rates}

Individuals who had published literature (on HHRA, incineration and public health, vulnerable populations), government HHRA/ toxicologist experts and other professionals in the academic field were contacted to be participants. A total of 71 experts were contacted via 
email with an invitation to participate. As mentioned, the interview package included an invitation letter as well as the interview template and research consent form to familiarize the individuals with the study and their role. From the 71 individuals contacted, 19 people responded back to the email. From the 19 individuals, 11 people stated that they were unable to participate either due to time constraints or due to conflicts of interest meaning $8 / 19$ individuals agreed to participate. According to Guest, Bunce and Johnson (2006), eight participants fall within the range of six to twelve interviews being sufficient with a narrow research scope. Miller (2012) stated that six to ten was a minimum number of formal interviews that can be conducted.

One individual stated that they were interested in participating and also consented to be a participant. After a few months of being out of town and a busy workload, the individual was unable to participate decreasing the total number of participants be seven. Out of seven participants, two required another ethics approval in addition to the Ryerson ethics approval in order to participate. Although it was a lengthy process, the second ethics board approved the project and allowed the two participants to take part in the study. Out of the seven individuals that participated, two took part in a face-to-face interview, two chose the telephone interview method and agreed to be recorded for note taking purposes, and three completed written email interviews due to the flexibility of the option. Although more interviews were hoped for during the process, the lack of response from the experts and the conflict of interest by others limited the number of participants. Due to time constraints of the research project, the Principal Investigator and the project supervisor came to the conclusion that seven would be a viable number of interviews for the project. It was assumed that this was not an issue since the 
interview method was used to support the primary method of data collection; the literature and risk assessment review.

Figure 3.2 shows the number and different specialization of participants who were contacted and Figure 3.3 shows the number and specialization of participants who consented to be part of the research project. It was hoped that there would be more individuals in each category but unfortunately, that was out of the researchers' control. It was apparent that many individuals declined due to supposed conflict of interest associated with the Durham-York EfW facility. It was determined that 5 of 19 who responded had a perceived conflict of interest stating that they were in some way affiliated with the Durham-York project, and could not participate due to that reason. This was unfortunate because the interview template did not require comments on the facility itself and was rather a template on general HHRAs focusing on vulnerable populations. The result was a participation rate of less than $10 \%$. The participants were given a random letter $A$ to $G$ to help referring to them throughout the study.

\subsection{Human Health Risk Assessment Framework}

After the literature and the HHRAs were analyzed, followed by the expert interviews, the process of creating the framework was initiated. This began by dividing the framework into five categories that were mentioned earlier. The first was the problem formulation stage, followed by the hazard identification stage, exposure assessment stage, dose-response stage, and finally the risk characterization stage. 


\section{Number and Specialization of Potential Participants}

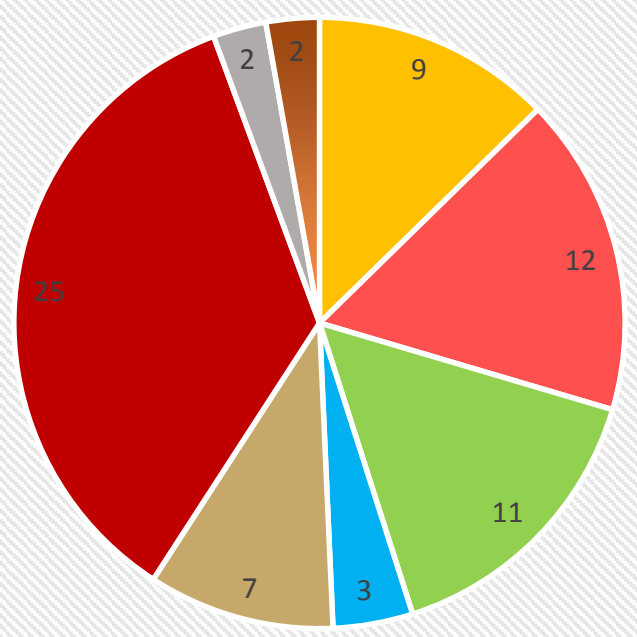

Risk Assessment Specialists

- Research Regulatory Toxicologists

- Epidemiologists

- Air Quality/Air Quality and Human Health Specialist

- Environmental Health Specialists

- Risk Assessment Consultants

- Environmental Science Specialists

Figure 3.2: Number and specialization of total individuals contacted

\section{Number and Specialization of Participants}

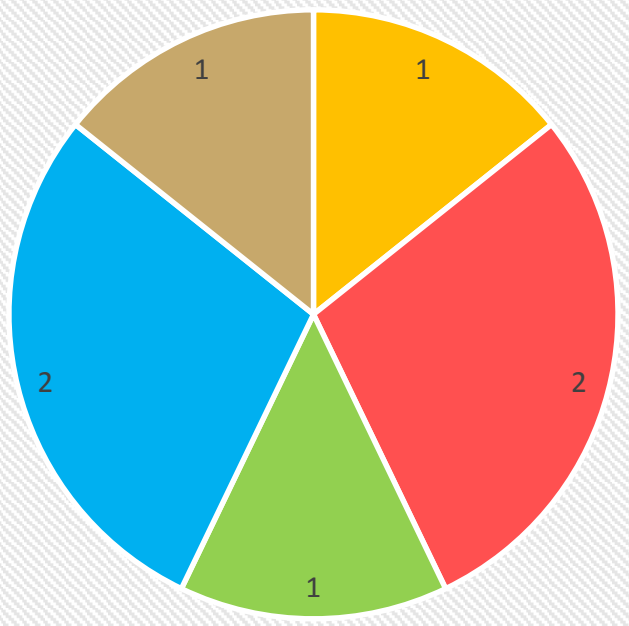

- Risk Assessment Specialists

- Research/Regulatory Toxicologists

Epidemiologists

- Air Quality/Air Quality and Human Health Specialists

- Environmental Health Specialists

Figure 3.3: Number and specialization of participants 
The Principal Investigator used an excel spreadsheet to organize different practices found in literature, previous HHRAs and interviews to the different stages of an HHRA. A number was given to each of the sources, including the interviewees to be able to identify their references when the final framework was created. Each source was reviewed a minimum of five times to ensure that all the information and best practices that were extracted were placed in the correct section and were truly a best practice. A criterion was identified a best practice by the number of times it was evident in different sources. If a requirement was not cited frequently, but was a crucial practice in authoritative sources, it was still included in the framework.

For the framework, $64^{5}$ sources were reviewed which included the interviews, HHRAs and the literature. The next section describes the different best practices that were placed in each risk assessment category, in detail. At the end of each section, the best practice criteria for each specific risk assessment category is presented, but the reference are omitted for sake of simplicity. The lists of references are lengthy, therefore, only one to two references are highlighted and the rest will be identified in the full framework (Appendix D) corresponding to the endnotes at the end of the document.

\subsubsection{Problem Formulation/Screening}

The problem formulation stage is the first step of the health risk assessment process. In many health risk assessments, this stage is not mentioned and is determined to be part of an

\footnotetext{
5 This number is in agreement with Snary (2002) who used 61 sources in his review of HHRA best practice criteria.
} 
initial screening process instead, but for the purpose of this research, and as observed in numerous HHRA guidelines (United States Environmental Protection Agency, 2004; Ontario Ministry of the Environment, 2005; Health Canada, 2012), it will be the first step of this framework. This stage presents initial screening of key components of the risk assessment and lays the foundation for the entire process, establishing whether the scope of the HHRA is adequate (United States Environmental Protection Agency, 2004; Government of Alberta, 2011).

There are many critical points to be met in the problem formulation stage for any risk assessment for EfW facilities. It begins with defining the problem and then determining whether the risk assessment will be a qualitative or a quantitative risk assessment (Ontario Ministry of the Environment, 2005). Focusing on the site characterization of the specific EfW facility, the location of the facility should be mentioned (Health Canada, 2012) along with the previous and current land use (Demidova and Cherp, 2005). This helps determine the type of area and the impacts of the waste facility in addition to different receptors it may affect. The problem formulation stage contributes information to the atmospheric dispersion model later on in the risk assessment, therefore the model used should be identified initially (Lonati and Zanoni, 2012). Identifying baseline environmental conditions is a significant best practice when assessing EfW facilities according to literature (Demidova and Cherp, 2005). Numerous interviewees felt that it was necessary in theory but difficult to do. In theory, establishing the existing chemical contamination level in environmental media is necessary to measure any deviations the facility will create from the baseline case. In practice, one expert commented that: 
[Determining baseline information] is expensive, so it should be considered carefully. This should be considered on a case-by-case basis and with careful consideration of how the baseline information will be used. For example, if your HHRA estimates an increase incidence of $1 / 10,000$ cancer risk over a lifetime and your worst-case scenario point of impingement will impact 100 people, then there is very little point to generating baseline health information for that impacted group (Interviewee B per. comm., 2014).

Another remarked:

It would be good to have as much baseline data [as] possible to complete the [Health Risk Assessment]. It would be important to know if any receptors would be predicted to have exposures above some standard like the [TRV] knowing the background exposures to the chemicals of concern would be useful in predicting how much the additional exposure from the facility will impact health risks (Interviewee A per. comm., 2014).

Interviewee F specified that "baseline information should be well characterized prior to the undertaking" (Interviewee F per. comm., 2014). Overall, the consideration of baseline information is important as explained by experts and as seen in the literature. Even though one expert stated that it be collected on a case-by-case basis, the inclusion of the baseline scenario is still considered a best practice.

The facility characterization in the problem formulation should include the air emission control technology to be employed by the facility to reduce the amounts of harmful contaminants it will potentially produce (Cangialosi, Intini, Liberti, et al., 2007). After the control technologies are identified, it is important to discuss the various contaminants ${ }^{6}$, including criteria air pollutants ${ }^{7}$ (Northeast Maryland Waste Disposal Authority, 2014) that can

\footnotetext{
${ }^{6}$ Refers to contaminants such a cadmium, arsenic, mercury, PAHs PCBs, dioxins and furans etc.

${ }^{7}$ Refers to contaminants such as carbon monoxide, sulfur oxides, nitrogen oxides etc.
} 
be released via the combustion process (United States Environmental Protection Agency, 2014e).

The central focus of this risk assessment framework is on pregnant women and fetuses, to determine the extent to which they are assessed in risk assessments. In this section of the risk assessment framework, it is crucial to determine the toxicity of the chemicals specific to life-stages (Daston et al., 2004) as different life-stages, particularly the fetal stage of development, experience different toxic effects of a chemical than an adult or a child. This also raises concerns of organ-specific toxicity for fetuses and for other young life-stages, so it is important to determine the organs or development systems that are affected by the chemical exposure and to consider specific time periods of concern (Daston et al., 2004).

Receptors that are susceptible to the chemical exposures should be identified in this stage as well, particularly focusing on which receptors are the most sensitive/vulnerable with a rationale of why they are considered vulnerable (Ginsberg et al., 2004). These populations are differentiated from the normal population based on numerous parameters including age, body weight, particular life-stages of development (fetus) or pre-existing life-conditions (Daston et al., 2004). An interviewee contributed a detailed description of the parameters in the following response:

We need to consider all the possible parameters that lead to increased risk of adverse effects. So each subpopulation would need to be looked at separately to determine critical factors that lead to susceptibility. Additionally the situation is made more complex because the mode of action ${ }^{8}$ differs with the different chemical exposures making the selection of key parameters more challenging. But overall, some key parameters would

\footnotetext{
${ }^{8}$ The term "mode of action" is defined as a sequence of key events and processes, starting with interaction of an agent with a cell, proceeding through operational and anatomical changes, and resulting in cancer formation (United States Environmental Protection Agency, 2005a)
} 
be related to the host-such as pharmacokinetics and pharmacodynamics (which themselves incorporate differences due to age, sex, body size etc.) as well as genetics. Additionally there are also parameters that impact exposures (i.e. children more likely to have exposures to contaminant in soil around their houses) (Interviewee A per. comm., 2014).

There are many more parameters that inform the definition of a vulnerable population, but for the purpose of this thesis, a pregnant woman and her fetus are the main focus.

Although the literature (Selevan et al., 2000; Olin and Sonawane, 2003; Daston et al., 2004; Ginsberg et al.,2004; World Health Organization, 2006; Brown et al., 2008; Cohen Hubal et al., 2008; Makris et al., 2008; Cohen Hubal et al., (2014) and the experts from interviews identified that pregnant women and fetuses are a vulnerable subpopulation to be investigated in a risk assessment, it was very difficult to find any risk assessments that mentioned pregnant women in their assessments of health risks. However, the Keeyask Generation Project Human Health Risk Assessment which is a hydropower project revised in 2013 was the only HHRA reviewed that considered pregnant women as a receptor group since mercury was a major contaminant of concern (Wilson Scientific Consulting Inc., 2013). This showed that although this segment of the population is highly sensitive, it was not a criterion that was commonly followed, but numerous literature agreed that it should be and therefore it was considered a best practice.

Another interviewee found that determining a vulnerable population "depends on science evidence about who is vulnerable, [whether from exposures] or a physiological way" but agreed that pregnant women and children should be among the vulnerable population considered in a risk assessment (Interviewee E per. comm., 2014). Another interviewee emphasized that pregnant women and fetuses should be assessed in a risk assessment because: 
There will be different toxicological endpoints. Also pregnant women's behavior (pica, eating and drinking more) and physiological changes can result in differences to biochemical parameters, update [and] excretion (Interviewee B per. comm., 2014).

One interviewee indicated that pregnant women and their fetuses should be assessed in a risk assessment with "the best available evidence and sure that the risks are properly assessed" (Interviewee A per. comm., 2014). They provided further insight stating:

The best practice is that a vulnerable subpopulation [such as the pregnant woman and fetus] would be considered separately from the larger population to ensure their risks are properly characterized (Interviewee A per. comm., 2014).

For receptors that are not considered in a risk assessment, a justification must be provided (Ontario Ministry of the Environment, 2005). This practice is an imperative part in the problem formulation stage as well as the entire risk assessment since the receptors determined here will be the only ones considered throughout the analysis. The assessor must recognize that even children have different life-stages ranging from the fetal to adolescent stage, therefore generalizations cannot be made. Simply referring to "children" as a receptor group cannot accurately describe the effects each different life-stage within the "children" category will experience (Daston et al., 2004; Ginsberg et al., 2004). Utilizing general terms will not allow an adequate protection of the fetus. Likewise, considering pregnant women as part of the "adult receptor" population is inadequate because inhalation of chemical contaminants not only harms the woman but harms the developing fetus and could cause impacts to both. Pregnant woman are found to exchange more air and have altered absorption or excretion patterns as well, and this impacts how they react to contaminants entering their bodies 
(Selevan et al., 2000). This stage should also identify the routes of uptake by these receptors, whether they be dermal, inhalation or trans-placental so greater detail could be provided for the specific pathways in the exposure assessment (Cohen Hubal et al., 2008).

The problem formulation stage should result in the development of a conceptual model which involves a qualitative characterization of the most relevant receptors (which life-stages are likely to be exposed), exposure/scenario pathways particular to the receptors identified, as well as patterns of exposures and endpoints of exposures (i.e. toxicity to organs) (United States Environmental Protection Agency, 2006; Cohen Hubal et al., 2008). The conceptual model helps in determining additional data that may be required to complete the risk assessment (Government of Alberta, 2011) and can be presented as a diagram, flow chart or a narrative description of the key relationships (United States Environmental Protection Agency, 2006). Exposure pathways that are not included in the problem formulation need to have a justification of why they were excluded (Ontario Ministry of the Environment, 2005).

Input from stakeholders and the public should be stated to give further insight as to how transparent and accessible the information was. Many of the interviewees felt that input from the stakeholders/public was necessary in all stages of the risk assessment. One stated that "the process should be transparent with the public being informed in all activities of the [HHRA]" and "they should have access to all [of] the information" in the risk assessment (Interviewee A per. comm., 2014). Another interviewee confirmed that the public/stakeholder input criterion should be a best practice because when they don't, issues arise (Interviewee C per. comm., 2014). An example was given by one interviewee referring to a cancelled gas plant. Interviewee D commented that "the public ran a muck and there was damage and costs 
financially because they did not involve the public [as] politicians perceived that it was not a concern to the public" (Interviewee D per. comm., 2014). Another participant stressed that this involvement of the public should be in an "early stage because acceptance at the end of the project is important" which supports why this criterion should be conducted initially, but exercised throughout the assessment as well (Interviewee E per. comm., 2014).

Overall, a risk assessment is hard to produce, and the use of previous risk assessments of the same type of project often serves as a guideline for new undertakings. Therefore, the use of any precedents (i.e. previous risk assessment calculations or exposure data from risk assessments/models/stack emission rates/contaminants of concern etc.) should be identified (Northeast Maryland Waste Disposal Authority, 2012). One of the interviewees stated that the use of precedents is important "to see if methodology is good" and if you are assessing a "similar population" (Interviewee E per. comm., 2014). One of the experts clarified the use of precedence stating that "it is not [important] in terms of setting of acceptable [exposure] levels or thresholds because knowledge changes" (Interviewee D., per. comm., 2014). One participant summed up the use of precedents well by commenting that "because [the assessments] are site-specific, they provide a guidance but do not become a precedent...if the precedent perspective is nice and complete, we [should] work towards that information" (Interviewee F., per. comm., 2014). This emphasizes that one should be careful when using precedents ensuring that they aren't repeated just because it has been working in the past. It is important to use them as a guideline and work towards producing new data as well. This best practice can be present in all the stages of the risk assessment, but since the problem 
formulation stage provides an overview of the data in the following sections, it was placed

here.

Table 3.1 below shows the best practice criteria for the problem formulation stage of a

HHRA. The best practices are presented in a checklist form for this and following sections but it

does not mean that the criterion will appear in the same order in the HHRA being evaluated.

Regardless of the order, the framework aims to assist a risk assessor though the process and

\section{Problem Formulation/Screening Best Practice Criteria}

1.1 The risk assessment uses a quantitative assessment of risk

1.2 Identifies the geographical location of the facility

1.3 Identifies land use

1.4 Identifies the baseline environmental conditions

1.5 Identifies the air emission control technology to be used

1.6 Identifies chemicals of concern that will be included in the risk assessment (prominently cadmium, mercury, arsenic, PAHs, PCBs, dioxin and furans)

1.6.1 Chemicals also include criteria air pollutants

1.6.2 Determines the toxicity of the chemicals specific to life-stages (sensitivity of receptors to chemicals)

1.6.3 Determines the organs/developmental systems that these chemicals may affect or if they cause any critical organ-specific toxicity

1.7 Identifies the preliminary receptors/potentially highly exposed life-stages (i.e. pregnant women) that are the most sensitive to the chemicals of concern identified and those more likely to be exposed

1.7.1 Defines vulnerable or highly exposed populations/life-stages using numerous parameters (i.e. age, body weight, fetuses, pregnant women, racialized communities, those with pre-existing health conditions etc.)

1.7.2 Provides a rationale for focusing on the particular groups of receptors/life-stages or why certain age groups experience higher exposures or are more susceptible

1.7.2.1 Specifically identifies fetuses/pregnant women as a vulnerable group

1.7.3 Detailed justification of any receptor/age group excluded provided

1.8 Identifies the most likely route of uptake of chemicals for different life-stages/receptors (gastrointestinal, absorption, dermal penetration, respiratory tract absorption/inhalation, ingestion trans-placental or a combination etc.)

1.9 Outlines a conceptual model to guide the risk assessment to consider sources, receptors (life-stage groups), stressors, exposure pathways/scenarios and individual characteristics of chemicals and endpoints (toxicity to organs etc.)

1.9.1 Provides justification for any omitted/incomplete routes or pathways

1.10 Allows for public/stakeholder input

1.10.1 Transparency of information is present

1.11 Discusses the use of any precedents (from other HHRAs) for data purposes

Table 3.1: A summary of the Problem Formulation/Screening best practice criteria 
recognize what is present and what is missing. When creating the criteria, some general HHRA practices were mentioned in all sections, but more emphasis was placed on criteria related to pregnant women and fetuses.

\subsubsection{Hazard Identification}

In the hazard identification step, data is evaluated for potential adverse health effects. It establishes whether exposure to a specific contaminant can cause an increased incidence of health effects in preconception, or other developmental life-stages (United States Environmental Protection Agency, 2006; United States Environmental Protection Agency, 2012).

In order to determine the adverse health effects to specific life-stages, the risk assessment must identify animal-based and human-based toxicological studies ${ }^{9}$ to determine the type and nature of the health effects from contaminants of concern (World Health Organization, 2010). It is assumed that when a chemical agent produces an adverse health effect in response to an exposure in experimental animals, humans have the potential of experiencing a reaction as well, but researchers are aware that the type of effects seen in animals does not accurately correspond to what will be observed in humans (World Health Organization, 2006). From all the research studies on animals and humans (if available), the risk assessment should weigh the evidence and reach a conclusion about the carcinogenic potential of the contaminants (United States Environmental Protection Agency, 2005a). These studies are used to establish evidence for identifying and characterizing the adverse events for a

\footnotetext{
${ }^{9}$ Should include human-based toxicological studies if they are available, but these are difficult to find due to ethical reasons.
} 
specific chemical agent and its chemical endpoints (Northeast Maryland Waste Disposal

Authority, 2012) as well as providing an evaluation under which conditions a hazard would be expressed (Snary, 2002). One interviewee described some of the chemicals released/produced by incinerators and their toxic health effects/endpoints to humans stating:

There will be exposures to fine particulate, lead, arsenic, acrolein, dioxins, furans, and other air toxics. There will be individuals with certain health conditions in which their disease/symptoms are worsened with exposure or who are susceptible to other adverse health effects. Individuals who have respiratory disease or functional deficits in respiratory function would be at increased risk. Also those who are immunocompromised may face higher risks. Also, since carcinogens will be emitted, those with genetic factors for increased cancer risk would be adversely impacted. Co-exposures to other carcinogens is a concern as well (Interviewee A per. comm., 2014).

Exposure to a developing fetus can have many different endpoints depending on the critical period of exposure (World Health Organization, 2006). This is why it is important to identify any life-stage specific health effects during the risk assessment process, making it easier to determine critical periods of development (Selevan et al., 2000). One interviewee went into detail with life-stage specific health effects to mercury, a contaminant released from the incineration process. They commented that "mercury is toxic to the developing brain [of the fetus $]^{10}$ and is transmitted across the placenta [from the mother to the fetus] (Interviewee E per. comm., 2014). The interviewee also commented that "[chemicals like] mercury, should be assessed differently for [pregnant women and fetuses] compared to the normal population, in a risk assessment" (Interviewee E per. comm., 2014) since the endpoints of these chemicals are very different for them compared to normal populations. These exposure effects can either

\footnotetext{
${ }^{10} \mathrm{~A}$ critical window of development in a fetus is the stage where the development of the brain occurs
} 
be immediate or have a later onset in life, therefore identifying that critical windows of development and the onset of the adverse health effects is crucial to the risk assessment (World Health Organization, 2006). Further, it is imperative to identify the most susceptible life-stages for exposure when discussing the harmful effects of contaminants (Brown et al., 2008).

Finally, the hazard identification stage should state the strengths and/or limitations of data used to support the weight-of-evidence ${ }^{11}$ and other conclusions, including data gaps and uncertainties (United States Environmental Protection Agency, 2006). Table 3.2 below summarizes the hazard identification best practice criteria.

\footnotetext{
Hazard Identification Stage Best Practice Criteria

2.1 Identifies animal-based and/or available human-based toxicology studies to determine the type and nature of health effects from contaminants of concern

2.1.1 Determines whether effects from animal studies are different than what humans will ultimately experience when extrapolating data from animals to humans and /or when extrapolating data from human adults to earlier life-stages

2.1.2 Determines weight-of-evidence to see whether chemicals may pose a carcinogenic risk 2.2 Provides characterization of hazardous/toxic effects or endpoints for each selected chemical (adverse effects/outcomes)

2.2.1 Identifies under which conditions the hazard can be expressed

2.2.2 Determines the life-stage-specific health effects/health outcomes (specifically for pregnant women where necessary)

2.2.3 Considers the potential for harm during critical windows of development and/or also considers delayed effects/late onset of chemical effects

2.2.4 Determines the most susceptible life-stages for exposure (i.e. pregnant women [fetuses] breast feeding infants)

2.3 States the strengths and /or limitations of data used to support the weight-of-evidence and other conclusions made in this step including data gaps and uncertainties
}

Table 3.2: A summary of the Hazard Identification best practice criteria

\footnotetext{
${ }^{11}$ In a situation of uncertainty, the term weight-of-evidence is used to describe whether evidence supporting one side of an argument is greater than that supporting the other side (Balls, Amcoff, Bremer et al., 2006).
} 


\subsubsection{Exposure Assessment}

The exposure assessment involves quantifying the level of chemical exposure per medium (i.e. air) to a receptor exposure route (i.e. inhalation, ingestion or dermal absorption) for complete exposure pathways (Entech-Rem Canada, 2013). Exposure assessments should identify potential pathways of exposure and quantify the magnitude, frequency and duration of human contact with a contaminant from various exposures (World Health Organization, 2006).

In detail, the exposure assessment should identify and characterize all receptors including vulnerable receptors and other highly exposed groups that were initially determined in the problem formulation stage (Ministry of the Environment, 2005; Health Canada, 2012). This includes determining the breathing rates, patterns of food consumption and other physiological changes for vulnerable populations (Selevan et al., 2000). Selevan et al. (2000) distinguished pregnant women from a non-pregnant woman as they found that pregnant women exchanged about $72 \%$ more air over 8 hours at rest which is 5,000 L compared to the $2,900 \mathrm{~L}$ in non-pregnant women. This difference is significant as greater air exchange implies breathing in more contaminants and this has the potential to cause detrimental effects to the fetus and the mother. Following this step, the receptor locations should also be identified in detail (Architecture, Engineering, Consulting, Operations and Maintenance, 2010) to determine the distance of the receptors from the incineration facility. This helps in determining who is getting the most/least exposure.

A significant contribution to the framework is the consideration of fetal and appropriate developmental life-stages having a higher intake-rate to body-weight-ratio. This is important to 
acknowledge since the higher intake-rate allows for greater exposures to a very small body weight, therefore greater impact on the body (World Health Organization, 2010).

The exposure assessment needs to identify possible pathways and different routes of exposure (Ontario Ministry of the Environment, 2005), particularly ones that are age/life-stage specific. Focusing specifically on the fetus, the exposure pathway of concern is the transplacental pathway (Cohen Hubal et al., 2008). A mother's exposure to contaminants suggests that she would become a significant source of contaminants to the fetus with transfer through the placenta (Cohen Hubal et al., 2008).

To characterize human exposures to toxic chemicals accurately, it is crucial to use the correct exposure factors ${ }^{12}$ that are adjusted to the relevant life-stage (fetus) when assessed. The EPA's Child-Specific Exposure Factors Handbook (United States Environmental Protection Agency, 2002) and the Exposure Factors Handbook: 2011 Edition (United States Environmental Protection Agency, 2011a) both recognize that pregnant women and fetuses have different body weights and characteristics compared to the normal population. These documents have adjusted factor values, which should be taken into consideration in HHRAs. For early lifestages, there are a series of critical windows of development, as mentioned earlier, and within these, exposure can be characterized differently depending on age-specific behaviours (United States Environmental Protection Agency, 2006).

\footnotetext{
${ }^{12}$ Some exposure factors include ingestion of drinking water, ingestion of fruits/vegetables, meat/dairy intake, human milk intake, dermal factors and human activity factors (United States Environmental Protection Agency, 2011a)
} 
The quantification of the chemical in different exposure media is an essential component of the exposure assessment stage. This concentration is what determines the exposures that humans will experience. This quantification leads to the dose-response stage of the risk assessment to estimate the intake rate/dose of the contaminant by the susceptible populations (Government of Alberta, 2011). This estimation of concentrations in different media is based on fate and transport modelling using air dispersion and air deposition models (CPF Associates, 2005).

Air dispersion models (i.e. California Puff Model (CALPUFF), American Meteorological Society/Environmental Protection Agency Regulatory Model (AERMOD) use data inputs such as physical and chemical properties of chemicals, bio-accumulation and bio-concentration factors for the relevant media (i.e. air, water, soil ) in addition to area-specific meteorological, hydrogeological and soil data properties (The Government of Alberta, 2011). Furthermore, the models require the concentration of a chemical in a medium and the time period in which there is contact between the individual and the chemical (World Health Organization, 2010). Air dispersion models vary from one another, but the model used must be widely received in the literature and adopted in practice. The name of the model should be mentioned in the exposure assessment section (World Health Organization, 2010). It is very important that the model used is well-recognized since justification for its use must be provided in the HHRA (The Government of Alberta, 2011) and it is important that the process for using the model is transparent and reproducible (The Government of Alberta, 2011). Both air dispersion and deposition modeling results provide the necessary information to calculate ambient air 
concentrations and deposition rates for selected chemicals of concern, which are then used in the fate and transport modelling (CPF Associates, 2005).

A discussion of start-up/shut-down/ accidental conditions is important because the different conditions result in the release of contaminants. One participant recognized that "start-ups and shut-downs are associated with higher releases and should be considered in the overall impact" (Interviewee C per. comm., 2014). Higher releases therefore contribute to higher exposures to different populations including vulnerable ones.

Finally, due to the modelling assumptions made in this section, there should be a discussion of the strengths and limitations of the model used for the fate and transport modelling (United States Environmental Protection Agency, 2005a). The exposure modelling is the core section of the exposure assessment therefore, it is imperative to ensure that the model is fully transparent (The Government of Alberta, 2011). The exposure assessment best practice criteria are summarized in Table 3.3 below.

\section{Exposure Assessment Best Practice Criteria}

3.1 Identifies and characterizes all receptors including vulnerable receptors and other highly exposed groups 3.1.1 Considers breathing rates, patterns of food consumption and other physiological changes for vulnerable populations

3.1.2 Identifies and evaluates receptor locations

3.1.3 Acknowledges a higher intake-rate to body-weight-ratio for fetus/children

3.2 Identifies various pathways and routes of exposure

3.2.1 Includes specific pathways of exposure to the fetus/young children via ingestion of breast milk/ trans-placental

3.3 Uses exposure factors that are adjusted for relevant life-stages of concern (i.e. fetus), if necessary

3.4 Provides the concentration of the pollutant in the exposure media

3.4.1 Identifies the air dispersion model (i.e. CALPUFF, AERMOD)

3.4.2 Identifies the results of deposition modelling

3.5 Presents a discussion of start-up/shutdown/accidental conditions

3.6 Discussion of strengths/ limitation/assumptions of the data and the models presented

Table 3.3: A summary of the Exposure Assessment best practice criteria 


\subsubsection{Dose-Response Assessment}

The dose-response assessment is also known as the toxicity assessment and it evaluates potential risks to humans at different exposure levels from the COPC (United States Environmental Protection Agency, 2005a). In general, this stage identifies the maximum dose humans can receive without an adverse effect (Cantox Environmental Inc., 2000). The doseresponse analysis is developed from studies that report quantitative data on the dose and its response (United States Environmental Protection Agency, 2005a).

In order to conduct a life-stage specific dose-response assessment, a careful examination of the critical periods of development should be documented, as exposure in these periods may result in more severe outcomes. Each life-stage is developmentally different, particularly the fetal stage. Theoretically, particular dose of a contaminant in the fetal life-stage will have different effects compared to the same dose in a different life-stage and these differences need to be acknowledged in a risk assessment. However, Howd (2010) reported that the fetus and infant would receive different doses of the same contaminant due to the differences in exposure routes, supporting that doses of a contaminant are dependent on lifestages.

Furthermore, it is important to identify different impacts on organ and biological systems in various susceptible life-stages in the dose-response assessment (Daston et al., 2004). Daston et al. (2004) described the critical developmental stages in the respiratory system, immune system and nervous system at different life-stages and this evaluation revealed that exposure during certain developmental periods can have a large impact on growth of an 
organism. Focusing on fetuses, critical periods in organogenesis and other fetal development stages are important to include, due to rapid growth during this time (Landrigan et al., 2004) and because the literature has associated exposures in this period with immune disorders and asthma as well (Daston et al., 2004). This analysis is necessary in order to understand the expected adverse effect for the specific dose received.

In order to characterize risks in a HHRA, TRVs need to be identified for each contaminant of concern and the pathway to which it will be applied (Health Canada, 2012). A TRV is a published values that is used in a risk assessment to characterize risks that are associated with chemical contaminants (Health Canada, 2010). Non-carcinogenic chemicals have a daily dose TRV that is an acceptable level whereas for carcinogens, the TRV represent an upper bound estimate of the slope of exposure and the effect, which is most probably cancer (Health Canada, 2010). For carcinogens, there is no exposure limit that is unlikely to produce an adverse health effect, as any exposure can contribute to cancer. Each TRV is different depending on the class of the chemical and the route of exposure-whether it is inhalation, ingested or dermal (Government of Alberta, 2011).

Any value that exceeds the TRV value for a chemical is expected to result in adverse effects specifically for non-carcinogens (Health Canada, 2012). The health effects that are associated with exceedances should be mentioned in this section whether they are developmental delays, low birth weight or others (United States Environmental Protection Agency, 2006). If a chemical is carcinogenic, the types of cancers that are a result of exposures to specific chemicals should also be identified here (Health Canada, 2012). Chemical-specific and/or population life-stage specific factors which include effects on the absorption, 
distribution, metabolism and/or excretion in vulnerable populations including pregnant women and fetuses should be identified (Olin and Sonawane, 2003). These are the factors that allow the assessment to characterize how toxic the chemical will be to the receptor in question (World Health Organization, 2006).

Each TRV is based on the duration of the exposure (whether it is acute, short-term of chronic) as well as the route of exposure, whether inhalation, dermal or oral (United States Environmental Protection Agency, 2006). These values are used for adults but they are said to be health protective for the most sensitive population as well, depending on the developmental life-stage and how it is derived for this subpopulation (United States Environmental Protection Agency, 2006). The source of the TRV values and the rationale for their use needs to be reported in this section as it is a common practice (Government of Alberta, 2011; Health Canada, 2012). Furthermore, the dose-response data used in this section to compare to the risks produced by the facility, must be obtained from a reliable source, including Health Canada, the US Environmental Protection Agency, California Environmental Protection Agency (CalEPA), or the Agency for Toxic Substances and Disease Registry (Government of Alberta, 2011; Health Canada, 2012). One expert agreed that the "USEPA and Health Canada" are reliable sources of dose-response data and are regularly used in Canada (Interviewee F per. comm., 2014).

Dose-response evaluation considers interspecies variability as well as human variability, but does not commonly incorporate exposures during specific life-stages (World Health Organization, 2006). Since the focus of this risk assessment framework is fetuses and pregnant women, adjustment/uncertainty factors are necessary with the availability of reproductive and development data to account for differences in variability (World Health Organization, 2006). 
This can be through extrapolations from animal studies (United States Environmental Protection Agency, 2006) to different critical windows of development. These adjustments would account for the differences in the receptors and provide an adequate evaluation of receptors to ultimately provide an accurate estimation of risks.

Furthermore, in order to appropriately assess health risks to all receptors, certain groups of chemicals need to be assessed as mixtures since mixtures have different effects on adults, children and various life-stages within children. There are different ways of assessing this including the analysis of additivity, antagonism, synergism and potentiation (Government of Alberta, 2011). Additivity is described as two more chemicals greater than the sum of the individual effects; antagonism occurs when one chemical reduces/blocks the effect of another chemical; synergism is when the combined toxicity of two or more chemicals is greater than the sum of the individual effects of each chemical, and potentiation is when one chemical increases the toxicity of another even if it is not toxic itself (Government of Alberta, 2011). This set of chemical interactions must be assessed carefully by the risk assessor.

It is best for a dose-response assessment to end with a narrative summarizing the section and uncertainties associated with dose-response values (United States Environmental Protection Agency, 2005a). The uncertainties may include the choice of experimental animal species that are used to derive dose-response values for humans or the extrapolation of available data to different human life-stages (United States Environmental Protection Agency, 2006) including fetal life-stages. The discussion of uncertainties is important because it has to account for the lack of data available for sensitive populations. Table 3.4 below summarizes the dose-response best practice criteria. 


\section{Dose-Response Best Practice Criteria}

4.1 Identifies the maximum dose humans can receive without any adverse effects or the relationship between the dose and the severity of the effect

4.2 Identifies susceptibilities of different life-stages for different biological/organ systems when exposed to chemicals (immune, nervous, respiratory, reproductive effects)

4.2.1 Identifies critical periods of fetal development and organogenesis to help identify dose-response relationships associated with chemicals

4.3 Identifies toxicity reference values (RfD, CSF, RfC, SF, URF, TDI, ADI)

4.3.1 Dose-response data obtained for at least one of the following reliable sources: obtained from at least one of the following reliable sources: MOE, Health Canada, USEPA, WHO, California EPA, ATSDR

4.3.2 Rationale provided of all exposure limits/toxicity reference values used

4.4 Identifies carcinogenic and non-carcinogenic endpoints for each chemical agent and for the different types of exposures

4.4.1 Identifies different health effects associated with the exposure to a chemical agent (i.e. cancer, mortality, developmental delays, birth defects, low birth weight)

4.5 Identifies chemical-specific and/or population/life-stage specific factors which includes in effects on pharmacokinetics (absorption, distribution, metabolism, and/or excretion)

4.6 Uses adjustment/uncertainty factors to account for specific receptors (i.e. fetal life-stage)

4.7 Evaluates possible chemical interactions of one or more of the following: additivity, antagonism, synergism, potentiation

4.8 Discusses significant uncertainties in the dose-response step

Table 3.4: A summary of the Dose-Response best practice criteria

\subsubsection{Risk Characterization}

The risk characterization stage is the final stage in the risk assessment process. In this stage, the integration of the problem formulation (Brown et al., 2008) hazard identification, exposure assessment and dose-response assessments occurs and allows for a quantitative estimate of health risks (Brown et al., 2008; Government of Alberta, 2011).

This information is then used by risk managers to make informed decisions about health risks to the populations of interest (United States Environmental Protection Agency, 2006).

Essentially, an estimate of risk is provided by comparing the exposure with the set guidance values (TRVs) (World Health Organization, 2010). The risk estimations that are developed must be in accordance with Canadian or International guidelines for safe exposure limits (i.e. Health Canada, USEPA, Integrated Risk Information System (IRIS), MOE, WHO) (Cantox Environmental 
Inc., 2000). In order to analyze the risks to specific chemicals, the dominant chemicals that may potentially affect different receptors must be identified (Cantox Environmental Inc., 2000) and any exceedances in these contaminant levels from the recommended exposure limits must be mentioned (Architecture, Engineering, Consulting, Operations and Maintenance, 2010).

The risk characterization takes the different exposure scenarios ${ }^{13}$ that are described in the initial parts of the risk assessment and creates a risk analysis (United States Environmental Protection Agency, 2005a). This is done by outlining the risks to the vulnerable population (Interviewee A per. comm., 2014; Interviewee B per. comm., 2014; Interviewee C per. comm., 2014; Interviewee D per. comm., 2014; Interviewee E per. comm., 2014) and comparing those risks to the other populations outlined in the problem formulation (World Health Organization, 2006) to establish the differences. It also puts the risks into perspective by comparing them to other types of risks (Cantox Environmental Inc., 2000). For example, some risk assessments compare a health risk being equivalent to travelling a specific speed in an automobile or plane, or even drinking a specified amount of liquid (Cantox Environmental Inc.,2000). This is meant to give people a greater understanding of the underlying risk estimates.

Furthermore, as in any general risk assessment, there are calculations that help determine whether exceedances of exposure exist for carcinogens and non-carcinogens.

\footnotetext{
13 "An exposure scenario considers the physical setting, potential uses of a contaminated resource (e.g., future residential land use or consumption of fish), the population that may be exposed (infant, child, or adolescent), fate and transport of contaminants, and how exposure may occur including ingestion, dermal contact, and inhalation" (United States Environmental Protection Agency, 2014f)
} 
For non-carcinogens, the hazard quotient ${ }^{14}$ is provided (United States Environmental Protection Agency, 2010) and many have included a hazard index calculation as well, which reflects the total chronic hazard which is attributable to exposure to all COPCs through a single exposure pathway (United States Environmental Protection Agency, 2005b) and is calculated by summing the hazard quotients for each exposure pathway and each chemical in that pathway (Electric Power Research Institute, 2013). For carcinogens, an excess lifetime cancer risk is compared to a de minimis level (i.e. $1 \times 10^{-6}$ or $1 \times 10^{-5}$ ). The de minimis level is an acceptable risk value for carcinogens when calculating the incremental lifetime risk (Health Canada, 2012). One participant commented on the de minimis level stating that one in a million is "appropriate, but not a standard and very stringent as well" (Interviewee F per. comm., 2014). The de minimis level can range from one in a million to one in one hundred thousand to even one in ten thousand depending on where (country or province) the risk assessment is being conducted. Another interviewee stated that this value was "conservative" in nature and that all populations "should be protected in one in a million" but that "this is an overestimate" (Interviewee G per. comm., 2014). The overestimation is to ensure that populations are protected.

A major part of the risk characterization step is uncertainty. An interviewee commented that:

\footnotetext{
${ }^{14}$ Hazard Quotients are values that are used to express risk from chronic exposures to non-carcinogens but through various exposure pathways (Jacques Whitford Limited, 2009c). A HQ of 0.2 is used in the Durham-York HHRA as a benchmark, therefore any intake of contaminants by all exposure routes less than 0.2 ensure that no adverse health effects are expected (Jacques Whitford Limited, 2009c). For methylmercury, a HQ of 1 is used because $100 \%$ of the methylmercury in a receptor would be coming from the ingestion of fish (Jacques Whitford Limited, 2009c).
} 
Best practice indicated the need to do sensitivity analysis to estimate the impacts of various assumptions on the overall estimate of risk. While a wide band of risk many be difficult to communicate, it is a good practice to be transparent about [the uncertainties]. This should also help indicate how protective the final estimates are (Interviewee $C$ per. comm., 2014).

A life-stage specific risk characterization should include a discussion of where data is deficient/unavailable and how this affects uncertainty in the risk estimates (Brown et al., 2008). Other uncertainties include the extrapolation of data from experimental animals to humans and from adults to earlier life-stages (i.e. fetal life-stage) since there is a difference in the toxicokinetics of both subpopulations (Brown et al., 2008). In addition, any uncertainties associated with life-stage specific adjustments (Brown et al., 2008) should also be mentioned. This involves the identification of any uncertainty factor used in the risk assessment to account for limitations and data gaps of information and specifically life-stage-specific uncertainty factors (United States Environmental Protection Agency, 2006). Assumptions made in different models and calculations used in the risk assessment should also be mentioned (Electric Power Research Institute, 2013) to develop a greater understanding of the risk estimates. An interviewee commented that "models are used in previous [risk assessment] stages (i.e. dispersion models for exposure assessment; PBPK models for dose) [and] each should be subjected to both sensitivity and uncertainty analysis" (Interviewee A., per. comm., 2014). The TRVs should be analyzed for accuracy (Ontario Ministry of the Environment, 2005) and determine whether they protect sensitive populations as well, which is likely done with the use of uncertainty factors (Health Canada, 2012). One participant stated that "we need better TRVs to indirectly better assess pregnant women and fetuses" (Interviewee F per. comm., 2014) 
to account for their sensitivities accurately. The strengths and limitations of all other data should also be presented here (Brown et al., 2008).

Looking at the entire risk assessment, not only does it have to be transparent, clear and concise, but it is important to assess the degree of confidence in the overall characterization as well (World Health Organization, 2006). If the risk assessment practices the precautionary principle ${ }^{15}$, it should be apparent when going through the risk assessment (Government of Alberta, 2011). An interviewee mentioned that the precautionary principle is already exercised since "the precautionary [approach is]... built into the 1 in a million" carcinogenic risk guideline (Interviewee D per. comm., 2014). One risk assessor commented similarly stating that the "precautionary principle is used [already]" (Interviewee G per. comm., 2014) but another stated that "it is difficult to use in the risk assessment because those who are oppose a project will use the precautionary principle to [stop it] not knowing that it already is used and lies within the professional judgment realm" (Interviewee F per. comm., 2014). Overall, it is important to note that the precautionary principle is significant as it protects human health in the face of uncertainty. The weight-of-evidence approach as well as public participation are both used in many risk assessments to incorporate the precautionary approach in risk assessments (McClenaghan et al., 2003).

\footnotetext{
${ }^{15}$ There is no one definition for the precautionary principle. According to the Rio Declaration on Environment and Development precautionary principle should be widely applied when possible and "when there are threats of serious or irreversible damage, lack of full scientific certainty shall not be used as a reason for postponing costeffective measures to prevent environmental degradation". This definition is more towards an environmental perspective. For this purpose of this research the definition has been adapted and the precautionary principle is when an activity raises threats or harm to human health or the environment and the precautionary approach is taken even in the face of scientific uncertainty (McClenaghan et al., 2003).
} 
The risk characterization should conclude by identifying any other critical data gaps and information that were not previously discussed in the stage as well as any future research needs to help perfect the risk assessment process for future risk assessments and help fill the gaps in knowledge to reduce the limitations in the future as well (Makris et al., 2008). Table 3.5 below displays the best practice criteria for the risk characterization stage of health risk assessments for vulnerable populations.

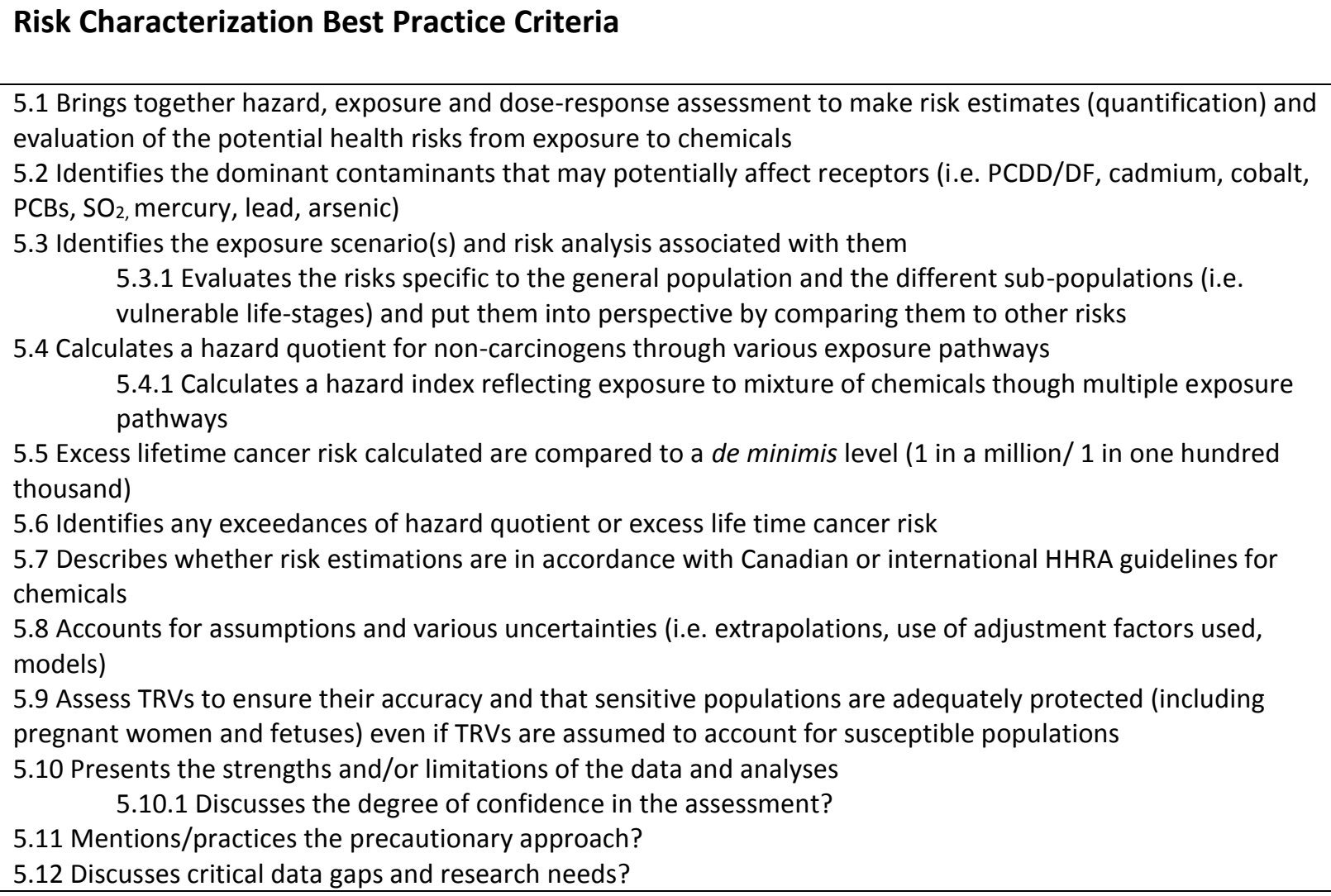

Table 3.5: A summary of the Risk Characterization best practice criteria

\subsection{Evaluation of the Durham-York Human Health Risk Assessment to the Best Practice}

\section{Criteria}

In order to fulfill the main component of the thesis, the Durham-York HHRA was

evaluated and compared to the best practice criteria to assess whether vulnerable populations, 
specifically pregnant women and fetuses were considered adequately. The best practice criteria served as a checklist when going through the HHRA but were not followed step-by step, since all HHRAs are conducted differently. First, the entire HHRA was reviewed and notes were taken in each section. The Durham-York HHRA was set up in a different format compared to the sections in the framework. The HHRA included a problem formulation stage, an exposure assessment a hazard assessment stage and finally a risk characterization stage. A distinct hazard identification stage was not included in the HHRA but the information was dispersed though different stages.

After reviewing the HHRA independently, it was examined using the evaluation checklist. $\quad A$ " $Y$ " for yes was entered for criteria that were considered/included in the risk assessment and an " $\mathrm{N}$ " indicated no for criteria that was absent in the risk assessment. Beside each " $Y$ ", the letter " $D$ " for deficient or " $C$ " for conforming with best practices, was entered. This identified if the criterion present in the risk assessment was fully compliant to best practices or was deficient and had limitations, even though it was present.

\subsection{Summary}

This chapter provided an overview of the method that was followed in this study. The chapter began with the methods used throughout the entire research process followed by an explanation of the qualitative research process including the interview template that was created, the recruiting process for the experts as well as their participation rates. The chapter then detailed the human health risk assessment framework and expectations in each stage 
when considering pregnant women and fetuses. The section then explained the process of how the framework/checklist was used to evaluate the Durham-York HHRA. The analysis is presented in the following chapter. 


\section{CHAPTER 4: RESULTS OF THE DURHAM-YORK HUMAN HEALTH RISK ASSESSMENT}

\subsection{Introduction}

This chapter provides an analysis of the Durham-York HHRA by comparing it to the best practice framework. Compliant, deficient and missing criteria are discussed for each stage of the HHRA separately for the problem formulation, hazard identification, exposure assessment, dose-response assessment and the risk characterization stage. The deficiencies and absence of criteria are the main elements of focus in this study.

\subsection{Problem Formulation/Screening: Conformities and Deficiencies}

The problem formulation section of the HHRA had criterion that conformed with best practices but presented several deficiencies as well. Table 4.1 presents criteria that conformed with best practices and Table 4.2 presents criteria that were considered in the HHRA but were deficient since they were missing important components. Finally, Table 4.3 will identify criteria that were absent. From the analysis, $12 / 20$ best practice criteria were met, $7 / 20$ were met with deficiencies and 1/20 was absent. A discussion of each category is provided below.

\subsubsection{Problem Formulation/Screening: Conformities with Best Practice Criteria}

In every risk assessment, it should be apparent whether a qualitative assessment of risk is being conducted or a quantitative assessment. The Durham-York HHRA mentions the use of a quantitative assessment to evaluate risks to human health. In situations where risks could not be evaluated quantitatively, they were discussed qualitatively (Jacques Whitford Limited, 


\section{Problem Formulation/Screening: Criteria that Conformed with Best Practices}

1.1 The risk assessment uses a quantitative assessment of risk

1.2 Identifies the geographical location of the facility

1.3 Identifies land use

1.4 Identifies the baseline environmental conditions

1.5 Identifies the air emission control technology to be used

1.6 Identifies chemicals of concern that will be included in the risk assessment (prominently cadmium, mercury, arsenic, PAHs, PCBs, dioxin and furans)

1.6.1 Chemicals also include criteria air pollutants

1.7 Identifies the preliminary receptors/potentially highly exposed life-stages (i.e. pregnant women) that are the most sensitive to the chemicals of concern identified and those more likely to be exposed

1.9 Outlines a conceptual model to guide the risk assessment to consider sources, receptors (life-stage groups), stressors, exposure pathways/scenarios and individual characteristics of chemicals and endpoints (toxicity to organs etc.)

1.10 Allows for public/stakeholder input

1.10.1 Transparency of information is present

1.11 Discusses the use of any precedents (from other HHRAs) for data purposes

Table 4.1: Criteria that conformed with best practices in the Problem Formulation/Screening stage of the Durham-York human health risk assessment

\section{Problem Formulation/Screening: Deficiencies of Best Practice Criteria}

1.6.2 Determines the toxicity of the chemicals specific to life-stages (sensitivity of receptors to chemicals) 1.6.3 Determines the organs/developmental systems that these chemicals may affect or if they cause any critical organ-specific toxicity

1.7.1 Defines vulnerable or highly exposed populations/life-stages using numerous parameters (i.e. age, body weight, breathing rate, fetuses, pregnant women, racialized communities, those with pre-existing health conditions)

1.7.2 Provides a rationale for focusing on the particular groups of receptors/life-stages or why certain age groups experience higher exposures or are more susceptible

1.7.2.1 Specifically identifies fetuses/pregnant women as a vulnerable group

1.8 Identifies the most likely route of uptake of chemicals for different life-stages/receptors (gastrointestinal absorption, dermal penetration, respiratory tract absorption/inhalation, ingestion trans-placental or a combination etc.)

1.9.1 Provides justification for any omitted/incomplete routes or pathways

Table 4.2: Deficiencies of best practice criteria in the Problem Formulation/Screening stage of the Durham-York human health risk assessment

\section{Problem Formulation/Screening: Absence of Best Practice Criteria}

1.7.3 Detailed justification of any receptor/age group excluded provided

Table 4.3: Absence of a best practice criterion in the Problem Formulation/Screening stage of the Durham-York human health risk assessment 
2009c).The HHRA discussed aspects of the general requirements in risk assessments adequately including information about the geographical location of the facility, as well as the air emission control technology to be used.

Baseline environmental conditions were present in the screening portion of the HHRA rather than in the Problem Formulation stage, however, both stages were assumed to be the same in the best practice framework. The baseline conditions included baseline concentrations in different medium such as soil, biota, as well as air (Ollson et al., 2014). The air medium evaluated did not include facility related emissions or exposures since this was evaluating the scenario prior to any construction (Ollson et al., 2014). The purpose of evaluating baseline conditions was to determine any existing health risks in the area from background exposures of contaminants.

In regards to air quality, a monitoring station was set up in the surrounding area of the proposed facility between September 2007 to December 2008 (Jacques Whitford Limited, 2009c). Criteria air pollutants were monitored as well as PAHs, metals and dioxins and furans. The risk assessment presented the results of the different contaminants monitored and how they compared to monitoring stations in other locations as well as ambient air quality criteria (Jacques Whitford Limited, 2009c). The Durham-York HHRA also included an analysis of baseline conditions plus conditions that would exist with the facility in the operational phase.

The Problem Formulation stage identified chemicals of concern that would be included in the risk assessment and this criterion is key. The contaminants were narrowed down by focusing on chemicals that were toxic, bioaccumulative, persistent and were viewed as a 
concern by regulatory authorities and the public (Jacques Whitford Limited, 2009c). The assessment analyzed criteria air contaminants as well as $\mathrm{NO}_{x}, \mathrm{CO}, \mathrm{PM}, \mathrm{NH}_{3}$, volatile organic compounds (VOCs), PAHs, metals and any other contaminant capable of causing environmental and health effects were included (Jacques Whitford Limited, 2009c).

The risk assessment also described the receptors that would be analyzed including receptors thought to be sensitive. These included five different life-stages: infants (0-6 months); toddlers ( 7 months to 4 years); children ( 5 years to 11 years); adolescents (12 year to 19 years) and adults (20 years to 75 years) (Jacques Whitford Limited, 2009c). Each were analyzed according to the differing land uses surrounding the facility (Jacques Whitford Limited, 2009c). These life-stages were then combined to create a "composite" receptor that would evaluate carcinogenic lifetime exposures for the following receptors: local resident (infant, toddler, composite), local farmers (infant toddler, composite), daycare/schools(toddler, adult), recreation user/sport (toddler composite), recreation user-camping (toddler, composite), additional exposures from swimming (toddler, composite) and additional exposures from hunting and angling (toddler, composite) (Jacques Whitford Limited, 2009c). The HHRA specified the toddler life-stage to be the most sensitive due to their lower body weight and their higher soil ingestion rates, therefore higher intake of contaminants (Jacques Whitford Limited, 2009c). Here, the best practice criterion of including sensitive receptors was met because the toddler life-stage was identified as being the most vulnerable. However, further analysis into this shows that pregnant women and fetuses were not considered amongst the vulnerable group although literature confirms that they in fact are vulnerable. This deficiency will be detailed in the next section. 
Furthermore, common routes of uptake were discussed in the assessment including inhalation, ingestion and dermal penetration in accordance with the appropriate media: soil, food agriculture (poultry, beef, eggs milk), as well as breast milk (Jacques Whitford Limited, 2009c). The problem formulation concluded with a detailed conceptual model outlining the risk assessment boundaries in a diagram format. The inclusion of public and stakeholder input in the risk assessment in addition to the transparency of the information, was essential. Meetings, open houses and other avenues for public discussion and engagement were held to receive public input in the initial stages of the risk assessment process and this was explicitly mentioned in the problem formulation section. Finally, the use of precedents from a previous risk assessment of a similar facility was mentioned. For the identification of contaminants of concern, data from the Algonquin Power Thermal Treatment Facility stack-testing was used in the risk assessment. This action was necessary seeing that the Durham-York facility was not built at the time the HHRA was conducted, therefore a facility that was similar was used to estimate future emissions.

\subsubsection{Problem Formulation: Deficiencies and Absence of Best Practice Criteria}

The Problem Formulation had several criteria that conformed to best practices but several deficiencies and absences were presented as well. For one, pregnant women and fetuses were not amongst the vulnerable receptors identified in the risk assessment and justification was not provided as to why they were excluded. The problem formulation stage should consider the most critical receptors and according to the literature (Selevan et al., 2000; Olin and Sonawane, 2003; Daston et al., 2004; Ginsberg et al., 2004, Brown et al., 2008; Cohen Hubal et al., 2008; Makris et al., 2008) this is a vulnerable group and should be identified in a 
HHRA especially for a facility that releases harmful emissions. In addition, Farlie (2007) recognized that pregnant women were a vulnerable population and extra precaution was necessary for their protection. Although this study was focused on nuclear power plants specifically tritium, his research revealed that exposures affect pregnant women differently than normal healthy adults and with the possibility of carcinogenic emissions from the DurhamYork facility, pregnant women are at risk. The Ontario MOE (2005) stated that the exclusion of any receptors must be justified and without a justification, a deficiency is recognized. Although the Durham-York HHRA identified an infant receptor ranged from 0-6 months old, it did not define what the zero years meant, and therefore it cannot be assumed that the fetus was considered. In addition, the parameters used to identify receptors were limited, focusing only on body weight, breathing rate and food consumption. According to Interviewee $E$ (Interviewee E per. comm., 2014) and the literature, those with pre-existing health conditions as well as those who are pregnant are also significant parameters to consider.

Specifically, the HHRA stated that the toddler was the most sensitive receptor, but the justification did not prove convincing, as pregnant women provide an additional source of exposure to the fetus trans-placentally, and this has the potential to cause more harm since pregnant women exchange more air than average women, and therefore more contaminants can directly be inhaled and affect the fetus and the mother.

The problem formulation identified exposure pathways that were considered in the risk assessment (ingestion, inhalation and dermal penetration) and provided justification for those that were not considered. However, due to the exclusion of pregnant women and fetuses, the trans-placental pathway was not considered either, and a justification was not provided. This 
pathway is important because a mother's exposure to environmental media can have direct effects on the fetus, via trans-placental transfer (Cohen Hubal et al., 2008). If it was argued that the zero in 0-6 months life-stage corresponded to an unborn fetus, the trans-placental pathway should have been taken into considered regardless. If not, a justification of why it was not included should have been presented. Neither case provides an explanation for the omission therefore presenting a deficiency.

There were two criteria that were mentioned in the risk assessment appendices, but were not included or discussed in the problem formulation section, which posed as a deficiency in the best practice criteria. One criterion was to include a preliminary discussion of the toxicity of the chemicals to receptors and another was to determine any organs or developmental systems that the pollutants would affect. Although this was discussed in the dose-response assessment, it would have been beneficial to highlight these in the problem formulation since

this section frames the rest of the risk assessment. An initial assessment of specific organs and how they may vary susceptibly during development would determine whether those organs or systems should be looked at in more depth when conducting the assessment.

\subsection{Hazard Identification: Conformities and Deficiencies}

The next section that was analyzed was the hazard identification stage. Table 4.4 presents the criteria that conformed to best practices, Table 4.5 outlines criteria that was met but deficient and Table 4.6 outlines the criteria that was absent in the HHRA. It is crucial to mention that the Durham-York HHRA did not have a labelled hazard identification stage in the 
risk assessment, but the information that was included was present into other stages of the document. These data were analyzed as part of the hazard identification stage. Out of nine best practice criteria in this stage, $7 / 9$ criteria were met, $1 / 9$ was met with deficiencies and 1/9 was absent.

\subsubsection{Hazard Identification: Conformities with Best Practice Criteria}

As previously mentioned, the Durham-York HHRA did not have a distinct hazard identification, but the HHRA toxicity profile (Appendix $\mathrm{H}$ of the Durham-York HHRA) included a majority of the information that would normally be part of this stage. Appendix $\mathrm{H}$ was present in the document to support the calculations of the TRVs in the dose-response assessment, which would later be used to determine whether risks were acceptable.

The toxicity profile provided detailed information on each contaminant of concern. Contaminants were listed in alphabetical order outlining animal or human-based studies that determined the toxicity of the specific chemical as well as the weight-of-evidence to support its carcinogenic potential. In addition, it is clear that animals and humans experience different effects at different doses of a chemical and that this should be accounted for in the risk assessment. This was recognized in the toxicity profiles with the use of uncertainty factors for any type of variation including interspecies variation. The toxicity profile divided the discussion of each contaminant into carcinogenic effects, if any, and non-carcinogenic effects. With this, a characterization of the toxic effects/endpoints of each chemical was outlined and made transparent to readers. This description also encompassed the conditions under which the hazard was expressed. For example, hydrogen chloride was described to be irritating and 


\section{Hazard Identification: Criteria that Conformed with Best Practices}

2.1 Identifies animal-based and/or available human-based toxicology studies to determine the type and nature of health effects from contaminants of concern

2.1.1 Accounts for whether effects from animal studies are different than what humans will ultimately experience when extrapolating data from animals to humans and /or when extrapolating data from human adults to earlier life-stages

2.1.2 Determines weight-of-evidence to see whether chemicals may pose a carcinogenic risk 2.2 Provides characterization of hazardous/toxic effects or endpoints for each selected chemical (adverse effects/outcomes)

2.2.1 Identifies under which conditions the hazard can be expressed?

2.2.2 Determines the life-stage-specific health effects/health outcomes (specifically for pregnant women where necessary)

2.2.4 Determines the most susceptible life-stages for exposure (i.e. pregnant women [fetuses] breast feeding infants)

Table 4.4: Criteria that conformed with best practices in the Hazard Identification stage of the DurhamYork human health risk assessment

\section{Hazard Identification: Deficiencies of Best Practice Criteria}

2.2.3 Considers the potential for harm during critical windows of development and/or also considers delayed effects/late onset of chemical effects?

Table 4.5: Deficiency of a best practice criterion in the Hazard Identification stage of the Durham-York human health risk assessment

\section{Hazard Identification Stage: Absence of Best Practice Criteria}

2.3 States the strengths and /or limitations of data used to support the weight-of-evidence and other conclusions made in this step including data gaps and uncertainties

*Excluded a hazard identification section

Table 4.6: Absence of a best practice criterion in the Hazard Identification stage in the Durham-York human health risk assessment

corrosive if in contact with any tissue; brief exposures were said to cause throat irritations

(Agency for Toxic Substances and Disease Registry, 2002). Although the term life-stage was not used in identifying health effects/outcomes, terms such as fetus, young infant or child was used to distinguish different stages of life considered in determining a hazard.

Each chemical identified in Appendix $\mathrm{H}$ recognized all the possible susceptible 
populations that it would affect, which was consistent with best practices. The list also identified pregnant women and the developing fetus as susceptible to many contaminants. Although it can be assumed that their sensitivity was considered in creating the TRVs used in the risk assessment to determine an acceptable level of exposure, it is uncertain.

\subsubsection{Hazard Identification: Deficiencies and Absences of Best Practice Criteria}

Evaluation of the HHRA identified that the exclusion of the hazard identification section is a critical absence from the best practice framework. Although hazard identification data was presented in other sections, particularly the dose-response section, the HHRA would have benefitted from a separate section for more clarity in the HHRA. The use of a hazard identification stage is a best practice in many risk assessments but sometimes a risk assessment may have a problem formulation instead of a hazard identification or vice versa. Sometimes, the hazard identification stage is merged with the dose-response section as well, which seemed to be the case in this HHRA. For the purpose of this discussion it was found that both stages should be present for a comprehensive risk assessment framework to analyze vulnerable populations.

Critical windows of development were considered in animal studies on pregnant rats, as doses of chemicals were given at different time periods of gestation (which varied by chemical) and their outcomes were analyzed. However, early life exposures and their effects in later lifestages were not considered and this presents a deficiency. Critical windows of development

such as a specific trimester or periods of gestation/organogenesis were not identified as having different physiological/cell structural effects as a result of chemical exposures. The HHRA could 
have benefitted with this information even if the data was limited because, this is a gap in knowledge that must be addressed in risk assessments (Brown et al., 2008). The timing and duration of the exposure has a particular effect across various life-stages (Brown et al., 2008). Furthermore, the criteria regarding a statement determining strengths and/or limitations of data used to support the weight-of-evidence and other conclusions and data gaps or uncertainties was not present in the HHRA. This omission occurred due to the exclusion of a hazard identification section in the HHRA.

\subsection{Exposure Assessment: Conformities and Deficiencies}

The Exposure Assessment stage was performed in accordance with best practice criteria, even though the assessment did not particularly identify pregnant women as a vulnerable population. Most criteria were met, with $10 / 13$ criteria conforming to best practices and $2 / 13$ being met but with deficiencies, with $1 / 13$ criteria absent. Table 4.7 presents the criteria that conformed to best practices whereas Table 4.8 highlights the criteria met with deficiencies. Table 4.9 highlights the criteria that was absent from the Durham-York HHRA.

\subsubsection{Exposure Assessment: Conformities with Best Practice Criteria}

Most of the exposure assessment conformed to best practices. The first criterion in the exposure assessment framework outlined the identification and characterization of all receptors including the vulnerable ones. This criteria was presented in the HHRA document and moved into the appendices for further detail. As mentioned earlier, pregnant women were 


\section{Exposure Assessment: Criteria that Conformed with Best Practices}

3.1 Identifies and characterizes all receptors including vulnerable receptors and other highly exposed groups 3.1.1 Considers breathing rates, patterns of food consumption and other physiological changes for vulnerable populations

3.1.2 Identifies and evaluates receptor locations

3.2 Identifies various pathways and routes of exposure

3.2.1(a)* Includes specific pathways of exposure to young children via ingestion of breast milk 3.4 Provides the concentration of the pollutant in the exposure media

3.4.1 Identifies the air dispersion model (i.e. CALPUFF, AERMOD)

3.4.2 Identifies the results of deposition modelling

3.5 Presents a discussion of start-up/shutdown/accidental conditions

3.6 Discussion of strengths/ limitation/assumptions of the data and the models presented

Table 4.7: Criteria that conformed with best practices in the Exposure Assessment of the Durham-York human health risk assessment

\section{Exposure Assessment: Deficiencies of Best Practice Criteria}

3.1.3 Acknowledges a higher intake rate to body weight ratio for fetus/children

3.3 Uses exposure factors that are adjusted for relevant life-stages of concern (i.e. fetus), if necessary

Table 4.8: Deficiencies of best practice criteria in the Exposure Assessment of the Durham-York human health risk assessment

* Indicates that this criterion was divided into two for the purpose of this evaluation since part of it conformed to best practices and the other part did not. They were identified as two separate criterion for evaluation purposes

\section{Exposure Assessment : Absence of Best Practice Criteria}

3.2.1 (b)* Includes specific pathways of exposure to the fetus/young children via trans-placental pathway

Table 4.9: Absence of a best practice criterion in the Hazard Identification stage in the Durham-York human health risk assessment

not among the vulnerable population characterized, but the life-stages considered in the risk assessment were examined in detail. There were five life-stages that were evaluated in the HHRA (infant, toddler or preschool child, child, adolescent and adult) in addition to a composite receptor that incorporated all the life-stages in order to assess potential lifetime cancer risk (LCR) and incremental lifetime cancer risk (ILCR) of individuals to a carcinogenic compound (Jacques Whitford Limited, 2009c). Detailed tables were provided in the Appendix G of the 
HHRA displaying physical and behavioural characteristics for each life-stage, which were then used to estimate the chemical exposure to each receptor. The source of the values identified was also provided. Some of the characteristics included breathing rates, body weight as well as food consumption rates depending on the type of receptor evaluated, and this satisfied a best practice criterion. The receptor locations were also included in the HHRA with a total of 309 identified including day cares, residential developments, schools, commercial locations as well as terrestrial and aquatic land uses (Jacques Whitford Limited, 2009c). The identification of the receptor locations was necessary to conduct a multi-pathway exposure assessment to evaluate chronic exposures through various media (Jacques Whitford Limited, 2009c) through an inhalation assessment.

In the problem formulation, it was mentioned that toddlers were the most sensitive receptor due to their lower body weights and the differences in their behavioural patterns such as their higher soil ingestion rates. This did not have to be re-iterated in the exposure assessment. The exposure assessment considered sensitive populations in their calculation of human exposures rates. For non-carcinogens, the calculations were conducted using the sensitive toddler receptor as opposed to any other receptor. For breast milk exposures, infants were appropriately used instead. The carcinogenic exposure rates were calculated for the composite receptors, defined earlier. Various exposure routes were identified in the exposure assessment of the HHRA. This included the breast milk exposure route which is a possible pathway of contamination for infants who breast feed. Although different pathways were identified, greater emphasis was put on the inhalation pathway. 
A central focus of the exposure assessment is to predict the rates of exposure of human receptors to different COPC through various exposure pathways and this is dependent on the concentration of pollutants in different exposure media. The Durham-York HHRA conducted air dispersion modelling to estimate the data for this since the facility was not yet constructed at the time of the HHRA. The fate and transport of the chemicals from the source and their persistence in the media were also conducted in the HHRA. Project-related emissions were predicted for ground level concentrations of contaminants using the CALPUFF dispersion model (Jacques Whitford Limited, 2009b) which was identified in the HHRA. A detailed explanation of the model and its selection was not described directly in the HHRA but references was made to the document Air Quality Assessment Technical Study Report (Jacques Whitford Limited, 2009b) that provided full details. Deposition of the contaminants into the environment was also calculated in various environmental media to estimate the health risks to different receptors from oral and dermal contact (Jacques Whitford Limited, 2009c) and this satisfied a best practice criterion.

The emission sources are necessary to evaluate in an exposure assessment. For this reason, the Durham-York HHRA presented process upset cases which included start-ups, shutdowns and any malfunctions in the incineration system. The HHRA listed the potential for malfunctions of different parts of the incinerator system (i.e. baghouse broken (torn) bags) followed by a description of the type of upset condition. Due to the lack of available data, emission rates for these scenarios were not mentioned in the document; however, the HHRA described another approach to estimate the emissions referencing the Human Health Risk Assessment Protocol for Hazardous Waste Combustion Facilities (United States Environmental 
Protection Agency, 2005b) and this approach was detailed in the HHRA. This discussion of startup, shutdown and upset conditions is important due to the abnormal emissions these events produce which result in additional exposures to humans. This best practice criterion was satisfied accurately.

Lastly, according to best practices, a discussion of strengths, limitations or assumptions should be presented for the data and models used. This criterion was satisfied adequately with limitations and assumptions identified throughout the exposure assessment. Assumptions were described where data were missing, and the uncertainties associated with the model chosen were also described. However, this was not done directly in the risk assessment, this was done in another document entitled Air Quality Assessment Technical Study ReportAppendix D (Jacques Whitford Limited, 2009b) that specifically discussed the CALPUFF air dispersion model, but this document was referred to several times in the exposure assessment.

\subsubsection{Exposure Assessment: Deficiencies and Absences of Best Practice Criteria}

A significant deficiency that was identified previously was the exclusion of transplacental pathways which in turn led to the absence of criteria 3.2.1 (b). This pathway was absent because pregnant women and fetuses were not considered as a vulnerable population of concern. The HHRA presented various exposure factors dependent on the receptors chosen. The HHRA divided up all the different receptors being evaluated and listed different exposure factors for each, whether it was the ingestion of farm grown fruits/vegetables, ingestion of agricultural products, direct inhalation (indoor/outdoor), ingestion/dermal contact with surface water, ingestion or local wild game/fish, as well as ingestion of breast milk. Appendix $G$ of the 
Durham-York HHRA presented comprehensive tables for each receptor and the exposure factors considered. The exposure factors were taken from the USEPA Exposure Factors Handbook, 2011 (United States Environmental Protection Agency, 2011a) which organizes the different factors by different age groups making it easy to select the correct values for calculation purposes. The HHRA identified gaps and discussed the lack of data available for every life-stage of concern and justified the use of different values and adjustments made for the exposure factors used. However, a deficiency was presented with the discussion of exposure factors. Various exposure factors were provided for different receptor groups, but again, due to the exclusion of the vulnerable subpopulation of concern, maternal and fetal related exposure routes were not presented in the exposure assessment.

Furthermore, a discussion of higher-intake to body-weight-ratio was already presented and the toddler was chosen to be the most sensitive. However, a deficiency exists in this criterion because fetal life-stage has a smaller body weight than toddlers and pregnant women inhale in greater amounts of air compared to the normal healthy female, therefore greater exposure occurs. Even though the toddler was discussed, all possibilities for intake to body weight ratios should have been considered in the HHRA.

\subsection{Dose-Response Assessment: Conformities and Deficiencies}

The dose-response assessment was identified as the hazard assessment in the DurhamYork HHRA. The exposure limits of the contaminants were identified by evaluating a dose and the corresponding response as well as effects of the dose. Out of the 12 best practice criteria, 
10/12 were met by the Durham-York HHRA and 2/12 was weakly met criteria. Tables 4.10 and

4.11 present the criteria that conformed to best practices and were deficient, respectively.

There were no criteria that were excluded in this section.

\section{Dose-Response Assessment: Criteria that Conformed with Best Practices}

4.1 Identifies the maximum dose humans can receive without any adverse effects or the relationship between the dose and the severity of the effect

4.2 Identifies susceptibilities of different life-stages for different biological/organ systems when exposed to chemicals (immune, nervous, respiratory, reproductive effects)

4.3 Identifies toxicity reference values (RfD, CSF, RfC, SF, URF, TDI, ADI)

4.3.1 Dose-response data obtained for at least one of the following reliable sources: obtained from at least one of the following reliable sources: MOE, Health Canada, USEPA, WHO, California EPA, ATSDR

4.3.2 Rationale provided of all exposure limits/ toxicity reference values used

4.4 Identifies carcinogenic and non-carcinogenic endpoints for each chemical agent and for the different types of exposures

4.4.1 Identifies different health effects associated with the exposure to a chemical agent (i.e. cancer, mortality, developmental delays, birth defects, low birth weight)

4.5 Identifies chemical-specific and/or population/life-stage specific factors which includes in effects on pharmacokinetics (absorption, distribution, metabolism, and/or excretion)

4.6Uses adjustment/uncertainty factors to account for specific receptors (i.e. fetal life-stage)

4.7 Evaluates possible chemical interactions of one or more of the following: additivity, antagonism, synergism, potentiation

Table 4.10: Criteria that conformed with best practices in the Dose-Response Assessment of the Durham-York human health risk assessment

\section{Dose-Response Assessment: Deficiencies of Best Practice Criteria}

4.2.1 Identifies critical periods of fetal development and organogenesis to help identify dose-response relationships associated with chemicals

4.8 Discusses significant uncertainties in the dose-response step

Table 4.11: Deficiencies of best practice criteria in the Dose-Response Assessment of the Durham-York human health risk assessment

\subsubsection{Dose-Response Assessment: Conformities of Best Practice Criteria}

This section of the HHRA determined the maximum dose that humans can receive without experiencing any appreciable health risk. The HHRA discussed the difference between 
threshold ${ }^{16}$ and non-threshold ${ }^{17}$ approaches when evaluating contaminants to estimate the toxicity profiles of chemicals and their effects on humans. The dose-response assessment had the majority of the toxicity information in Appendix H-The HHRA Toxicity Profiles, which was cited throughout the section. Here, various life-stages were identified as being susceptible to different contaminants specifically to different biological/systems when exposed. For example, literature found that lead has the ability to produce neurological effects on the child life-stage and that nitrogen dioxide has the ability to increase the incidence of acute respiratory illness in children (Jacques Whitford Limited, 2009d). For the elderly life-stage, it was found that due to declining organ function, they were more susceptible for further organ damage due to mercury exposure (Jacques Whitford Limited, 2009d).

Pregnant women and the fetal life-stage were considered a susceptible population when determining the toxicity of chemicals and appropriate TRVs. Pregnant women were found to be highly susceptible to a variety of contaminants, ${ }^{18}$ more than an average healthy adult. For some chemicals such as tetrachloroethane, the nervous system of the developing fetus was particularly susceptible (Jacques Whitford Limited, 2009d). The fetus was found to be susceptible if exposed to mercury due to immature and developing organs (Agency for Toxic Substances and Disease Registry, 1999 as cited in Jacques Whitford Limited, [2009b]).

\footnotetext{
${ }^{16}$ Threshold dose-response refers to a benchmark that must be exceeded in order for toxicity to occur and corresponds to non-carcinogens (Jacques Whitford Limited, 2009c)

${ }^{17}$ Non-threshold dose-response refers to the inability to identify a specific dose where the health effects start to occur and corresponds to carcinogenic contaminants (Jacques Whitford Limited, 2009c)

${ }^{18}$ Contaminants included but not limited to pentachlorophenol (PCP), polychlorinated biphenyls (PCBs), vanadium, xylenes, carbon monoxides, non-criteria air contaminants such as 1,1-dichloroethene, barium, ethylbenzene, lead, mercury, tetrachloroethane (Jacques Whitford Limited, 2009d)
} 
The goal of Appendix $\mathrm{H}$ was to derive the TRVs which are doses of chemicals or benchmarks that receptors could be exposed to without developing harmful health effects (Jacques Whitford Limited, 2009c). The HHRA also identified different terms that are used in place of the TRV as they vary by jurisdiction (i.e. reference dose (RfD), reference concentration (RfC), cancer slope factor (SF), unit risk (UR) (Jacques Whitford Limited, 2009c). The HHRA presented a summary of the non-carcinogenic and carcinogenic TRVs used in both the inhalation multi-pathway exposure assessment for the criteria air contaminants and contaminants of concern. Oral TRVs were also presented where data were available. The source and rationale for each TRV used was also identified. Sources ranged from the Ontario MOE, Health Canada, IRIS, ATSDR, Canadian Council of the Ministers of the Environment (CCME), WHO, CalEPA, Texas Commission of Environmental Quality (TCEQ) as well as Alberta Environment (AENV) (Jacques Whitford Limited, 2009c). The rationales for each TRV used were described in detail in Appendix $\mathrm{H}$, using animal and human experiments to derive the appropriate values. Many of the laboratory experiments used pregnant mice as the sensitive population to derive the TRVs. In addition to the derivation, the carcinogenic and noncarcinogenic endpoint of each chemical agent was identified in the HHRA as well as Appendix $\mathrm{H}$. Different chemicals had different effects associated with them, whether it was low fetal weight $^{19}$ or cancer with exposure to arsenic, or birth defects associated with exposure to 1,1dichloroethane, beryllium, and polycyclic aromatic hydrocarbons (PAHs), to name a few.

Wherever data permitted, pharmacokinetic data and modelling were used in order to

\footnotetext{
${ }^{19}$ Low fetal weight is for mice, since human studies are unethical in this case ( Jacques Whitford Limited, 2009d)
} 
help derive the TRVs. Data for cadmium permitted the calculation of pharmacokinetic parameters of cadmiums' absorption, distribution, metabolism and its excretion and this resulted in a higher confidence level of the oral TRV calculated (Jacques Whitford Limited, 2009d). For other contaminants such as lead, lack of data permitted the exclusion of pharmacokinetic data to estimate the oral carcinogenic risk of lead (Jacques Whitford Limited, 2009d). Determining the fate of the contaminant using pharmacokinetics is a crucial part of the dose-response assessment and was considered in the derivation of health-based TRVs in the HHRA. The HHRA highlighted oral and dermal bioavailability factors to account for the amount of chemical that reaches either the bloodstream after ingestion or after it has come in contact with skin (Jacques Whitford Limited, 2009c). Where dermal bioavailability factors were not present, default values from the USEPA provided guidance (United States Environmental Protection Agency, 2008b). Most of the contaminants were evaluated via the inhalation pathway as it was assumed to be the primary pathway to exposure. For this reason oral and dermal absorption factors were not determined.

Interspecies and Intraspecies variations were highly common in TRVs due to the animal and some human studies conducted. Since receptors differ by life-stages or vulnerability, the use of adjustment/uncertainty factors is necessary to ensure that the TRVs are applicable to the receptor. The Durham-York HHRA used 10-fold uncertainty factors to account for various extrapolations to derive a TRV (Jacques Whitford Limited, 2009d). The uncertainty factors accounted for different extrapolations made from animal to human or for the sensitivity of an individual towards a specific contaminant (Jacques Whitford Limited, 2009d). In addition, 10fold uncertainty factors were used for some TRV calculations wherever there was unavailability 
of data. Wherever uncertainty factors were used, a description identified what each 10-fold factor accounted for.

Many HHRAs analyze more than one contaminant of concern, therefore, it is important to consider the potential for toxic effects of more than one chemical interacting with others. Chemical interactions would have different effects than a chemical by itself (Government of Alberta, 2011). The Durham-York HHRA assessed the carcinogenic PAHs as a mixture and the potency was based on the most toxic known PAH, benzo[a]pyrene (Jacques Whitford Limited, 2009c). A discussion was also presented on the combined effects (additivity) of chemicals which interact and a table was presented displaying health endpoints of mixtures and the chemical mixtures that can produce it. Potential interactions of chemicals were found to cause many different effects, particularly developmental and reproductive effects.

\subsubsection{Dose-Response Assessment: Deficiencies of Best Practice Criteria}

There was one deficient criterion identified in the Durham-York HHRA and it was concerned with critical windows of development. There were only a few cases where exposure during critical periods of development were tested. For example, in animal toxicity studies, rats were given various doses of mercury in gestation days 11-14 and 17-20 which corresponded to changes in behavior and learning disabilities, depending on the dose (Jacques Whitford Limited, 2009d). Arsenic was exposed to pregnant mice on gestation days 9-12 for 4 hours and this resulted in decreased fetal weight in the mice (Jacques Whitford Limited, 2009d). It is crucial to base the TRVs on this type of data because it is clear that the various windows of development for different life-stages have different effects depending on the type of exposure. The timing 
and duration of exposure is critical in all cases, particularly for pregnant women and fetuses. A complete discussion outlining the significance of various critical windows and periods of organogenesis in addition to effects of exposures during windows in fetal development, was necessary but not present. Most chemicals had the fetus and pregnant women as a susceptible life-stage but only a few explicitly considered them in the calculation of the TRV for that chemical. If TRVs are assumed to be protective of all life-stages, then consideration should have been apparent for each TRV calculated.

The dose-response assessment is associated with numerous uncertainties and calculations. Therefore, a separate summary of uncertainties pertaining to the dose-response stage would have benefitted the discussion, but was not presented. Nevertheless, a few uncertainties were discussed, which is why this was treated more as a deficiency rather than an exclusion. A summary of the uncertainties would have been helpful to provide an overview of what was uncertain in the data and therefore provide transparency for the readers.

\subsection{Risk Characterization: Conformities and Deficiencies}

The risk characterization stage is the final stage of the risk assessment process and is where the decision is made whether the predicted risks are acceptable and whether the facility is likely to produce health effects. There were a total of 15 best practice criteria in the risk characterization section of the framework of which $12 / 15$ conformed to best practices, 2/15 which deficient, and 1/15 that was absent. Tables 4.12, 4.13 and 4.14 show the criteria that conformed to best practices, were deficient and were absent, respectively. 


\section{Risk Characterization: Criteria that Conformed with Best Practices}

5.1 Brings together hazard, exposure and dose-response assessment to make risk estimates (quantification) and evaluation of the potential health risks from exposure to chemicals

5.2 Identifies the dominant contaminants that may potentially affect receptors (i.e. PCDD/DF, cadmium, cobalt, $\mathrm{PCBs}, \mathrm{SO}_{2}$, mercury, lead, arsenic)

5.3 Identifies the exposure scenario(s) and risk analysis associated with them

5.4 Calculates a hazard quotient for non-carcinogens

5.4.1 Calculates a hazard index reflecting exposure to mixture of chemicals though multiple exposure pathways

5.5 Excess lifetime cancer risk calculated are compared to a de minimis level ( 1 in a million/ 1 in one hundred thousand)

5.7 Describes whether risk estimations are in accordance with Canadian or international HHRA guidelines for chemicals

5.8 Accounts for assumptions and various uncertainties (i.e. extrapolations, use of adjustment factors used, models)

5.10 Presents the strengths and/or limitations of the data and analyses

5.10.1 Discusses the degree of confidence in the assessment

5.11 Mentions/practices the precautionary approach

5.12 Discusses critical data gaps and research needs

Table 4.12: Criteria that conformed with best practices in the Risk Characterization stage of the DurhamYork human health risk assessment

\section{Risk characterization Stage: Deficiencies of Best Practice Criteria}

5.6 Identifies any exceedances of hazard quotient or excess life time cancer risk

5.9 Assess TRVs to ensure their accuracy and that sensitive populations are adequately protected (including pregnant women and fetuses) even if TRVs are assumed to account for susceptible populations

Table 4.13: Deficiencies of best practice criteria in the Risk Characterization stage of the Durham-York human health risk assessment

\section{Risk Characterization Stage: Absence of Best Practice Criteria}

5.3.1 Evaluates the risks specific to the general population and the different sub-populations (i.e. vulnerable lifestages) and put them into perspective by comparing them to other risks

Table 4.14: Absence of a best practice criterion in the Risk Characterization stage of the Durham-York human health risk assessment

\subsubsection{Risk Characterization: Conformities with Best Practice Criteria}

A significant step in the risk characterization stage is to bring together the previous stages of the HHRA which include the hazard, exposure and dose-response assessment to make 
risk estimates (quantification) and to evaluate the potential health risk from exposures. These risk estimations are completed when the predicted intake of the contaminants is compared to the TRVs which were derived in the dose-response assessment. For non-carcinogens, the Durham-York HHRA acknowledged that human exposure can occur through various sources and not only air. For this reason, the HHRA decided to set a concentration ratio ${ }^{20}$ (CR) benchmark value of 1.0 as CR values are only applicable to air and $100 \%$ of exposure would occur in this pathway. On the other hand, HQs are associated with assessments that have multiple routes of exposure. In this case, with five potential sources of exposure (air, soil, water, produce, agricultural products), the $\mathrm{HQ}$ value was set at 0.20 . The hazard quotients and concentration ratios were calculated for the appropriate chemicals and exposure pathways in the risk assessment.

The risk characterization stage was divided into four assessment scenarios in order to effectively evaluate all possible health risks and included the baseline scenarios, construction scenarios, operations scenarios and finally the decommissioning scenarios (Jacques Whitford Limited, 2009c). The operations scenarios were evaluated at the design capacity of 140,000 tpy and a maximum design capacity of 400,000 tpy to ensure all possible operating conditions were considered (Jacques Whitford Limited, 2009c). Each scenario included different cases that were also analyzed.

In each of the assessment scenarios, tables were created identifying the contaminants

\footnotetext{
${ }^{20}$ Concentration ratios are used to evaluate health risks (acute and chronic) which are a result of exposure of chemicals in air (Jacques Whitford Limited, 2009c). A concentration ratio of 1 was used in the HHRA because exposure in the inhalation assessment has only one exposure pathway which is through air. For this reason, the entire toxicity reference value can be used here (Jacques Whitford Limited, 2009c)
} 
of concern and their concentration ratio/hazard quotient values, where relevant. The tables also identified which contaminant or contaminant mixtures exceeded the benchmarks used in the risk assessment. This satisfied the criterion for identifying contaminants that had the potential to cause harm, as well as the criterion requiring the identification of exposure scenarios and their risk estimates. The CR or the HQ's that were calculated showed whether a benchmark was exceeded or not, following the guidelines CR benchmark value of 1 and the HQ value of 0.2. Chemical mixtures were also evaluated for risks with their corresponding risk values. It was ensured here that vulnerable populations were the focus in the risk assessment, since the risks being calculated were for either the infant receptor or toddler receptor and all the exposure scenarios included either a toddler or infant where appropriate.

For non-threshold carcinogenic contaminants, a hypothetical cancer risk was assumed. For this reason, an incremental lifetime cancer risk of 1 in 1,000,000, also known as the de minimis level, was assumed. 1 in 1,000,000 was used instead of 1 in 100,000 solely due to the province the project took place in. Ontario commonly uses the stringent de minimis value of 1 in $1,000,000$ (Ontario Ministry of the Environment, 2005). Any carcinogenic calculations that exceeded this benchmark used in this assessment indicated that there was an elevated level of risk for the contaminant in question (Jacques Whitford Limited, 2009c). Wherever the risks of carcinogenic contaminants were presented in the HHRA, they were compared to the de minimis level in each scenario.

The risk assessment presented an uncertainty analysis with reasonable assumptions in most of the document. In addition, it overstated risks and acknowledged the limitations of risk assessment conclusions. According to the risk assessment, this produces a document with a 
greater level of confidence, therefore, this criterion was satisfied. The discussion of uncertainties was present in a clear and concise manner for readers. The major assumptions used in the risk assessment were presented in a table divided into uncertainties that were associated with the exposure assessment, dose-response assessment as well as the risk characterization stage. The table also presented a discussion of the conservative nature of assumptions and whether the assumptions overestimated/underestimated the risk or whether it was neutral. The uncertainty discussion included assumptions made for models, the use of the most sensitive age group, the use of current toxicological data, the derivation of TRVs, the use of the target $\mathrm{HQ}$ values of 0.2 , the Incremental lifetime cancer risk set to 1 in $1,000,000$, just to name a few.

A discussion of any strengths and/or limitations is significant for the transparency of a risk assessment. Limitations associated with chemical mixtures were identified in this section. It was determined that there are currently no regulatory benchmarks for chemical mixtures which makes it difficult to evaluate them (Jacques Whitford Limited, 2009c). In addition, there is a small probability of each contaminant in a chemical mixture being present in one location at the same time as the receptor in the same location and time (Jacques Whitford Limited, 2009c). Even with these uncertainties, chemical mixtures were considered in the risk assessment. Furthermore, various literature studies were used to support the use of a TRV, therefore satisfying a criterion. The different regulatory agencies and their documents were referred to in the HHRA when data was presented.

Ironically, for the hazard index calculation, the exclusion of the calculation was a strength in the risk characterization stage. The USEPA (2011b) defines the hazard index as a 
sum of the hazard quotients for chemical mixtures that affect the same target organ and have similar effects. The position taken in the Durham-York HHRA was that the hazard index calculation assumed that health effects of the contaminants were additive, therefore disregarding various toxicological endpoints of contaminants (Jacques Whitford Limited, 2009c). Therefore, the HHRA followed the USEPA (2005b) method of segregating the hazard index by summing the contaminant-specific hazards according to similarities such as the same target organ or systems they affect. For calculation purposes, if any of these values exceed the target hazard level assumed in the risk assessment, there would be a possibility of adverse effects (United States Environmental Protection Agency, 2005b). Although there was an exclusion of the chemical mixture calculation requiring the addition of hazard quotients, the individual organs and biological systems and their HQs for the different receptors were presented in table format and the justification provided was from a credible source. Due to the limitation of data, the hazard index segregation or calculating the hazard index can both be used and are considered to be part of the same best practice.

Furthermore, the risk assessment exercised the precautionary principle in many different parts of the risk assessment. The weight-of-evidence approach and public participation opportunities were outlined throughout the HHRA process and both are tools used to incorporate the precautionary approach (Canadian Environmental Law Agency, 2000) in the face of uncertainty. In addition, the precautionary approach was inherent in the TRVs with the use of uncertainty factors. Full certainty was absent for the contaminants that were analyzed but they were still considered in the risk assessment, exercising the precautionary approach. In addition, it was clear that lack of data did not prevent the HHRA from considering 
health risks from chemical mixtures, or specific data pertaining to certain receptors. This satisfies the precautionary principle as a lack of full scientific uncertainty was not an excuse for excluding receptors, chemicals or any other data from the risk assessment (Canadian Environmental Law Agency, 2000).

Finally, the HHRA risk characterization satisfied the best practice criterion of discussing critical data gaps, therefore identifying research needs. As mentioned earlier, the lack of data pertaining to chemical mixtures is a considerable source of uncertainty in risk assessments. The uncertainty analysis also mentioned the limitations of toxicological data when deriving TRVs, which also presents a great degree of uncertainty. This directly considers the need for greater research on chemical mixtures so that this gap in data can be filled.

\subsubsection{Risk Characterization: Deficiencies and Absences of Best Practice Criteria}

Analysis of the HHRA identified a few deficient criteria, particularly the one concerning exceedances of contaminant levels. Although $\mathrm{HQ}$ values, concentration ratios and excess life time cancer risks revealed exceedances, the justification for those exceedances were deemed inadequate. The risk assessment identified exceedances of PCBs, dioxin and furans, arsenic and various other contaminants particularly for infants and toddlers beyond regulatory values. The justifications provided were similar in most cases stating that most exceedances was a result of the baseline case and not the project itself. Regardless of where they stemmed from, construction of the facility would result in a greater exceedance of contaminants into the atmosphere, therefore compromising public health. Instead, the risk assessment needed to address ways of decreasing the levels of contaminants rather than attributing blame to baseline 
cases. If baseline cases were already elevated from regulatory levels, perhaps the location of the facility was not the best option. Data showed that the highly sensitive toddler receptor was experiencing many of the exceedances along with the infant indicating that the vulnerable population is at risk.

The uncertainties outlined in the risk characterization section provided a discussion about the TRV used. It stated that conservative assumptions were adopted to account for interspecies variation, individual differences, limitations in the toxicological information and extrapolations from acute to chronic exposures (Jacques Whitford Limited, 2009c). It also stated that these assumptions were conservative in nature, therefore, resulting in an overestimation of risk (Jacques Whitford Limited, 2009c). However, a discussion on whether the TRVs were accurate for the most sensitive population would have made the risk characterization stage stronger. Although Appendix $\mathrm{H}$ in the HHRA presented the TRV derivation and detailed information on this, a discussion in the uncertainty analysis would have presented a stronger overview of the uncertainties for readers to be aware of.

There were a few exclusions that should be noted a well. The criterion seeking the evaluation of risks specific to the general population and how they compare to risks to vulnerable life-stages would have made the risk communication easily understandable for readers. In addition, comparing these risks to general risks such as car accidents, plane accidents, or any other type of risk could have also aided in understanding what the risk estimates mean, offering re-assurance to the public of what the risks really are. Relying solely on numerical values of risk has limited meaning to the public in the absence of a qualitative comparison. 


\subsection{Summary}

Overall, this chapter provided an in-depth analysis of the Durham-York HHRA evaluation by specifying the conformities, deficiencies and absences of each section. This was done by using the best practice framework that was presented in Chapter 3. The final chapter will present a discussion and present conclusions of the findings, determining whether the Durham-York HHRA conformed to best practices and adequately considered pregnant women and fetuses. 


\section{CHAPTER 5: DISCUSSION AND CONCLUSIONS}

\subsection{Introduction}

This fundamental purpose of this research is to determine whether the Durham-York HHRA was conducted with adequate consideration of pregnant women and fetuses and whether it conformed to best practices. This is meant to fill the gap in the health risk assessment practice and to protect vulnerable populations that are highly susceptible to environmental pollutants. This chapter will provide a discussion to the results found in the previous chapter with a complete evaluation of the findings. The chapter will then revisit the research objectives and will assist in providing an overview of the research conducted and major findings from the analysis. Study limitations that were encountered will be discussed and will be followed by a discussion of future research goals.

\subsection{The Complete Evaluation of the Durham-York Human Health Risk Assessment and its Compliance to Best Practices}

The conformities and deficiencies of the best practice criteria coincided with how adequate the Durham-York HHRA was conducted. This section of the chapter will discuss the results and determine whether vulnerable populations were adequately considered in the Durham-York HHRA.

The HHRA best practices framework facilitated the analysis of whether the Durham-York HHRA conformed to best practices. In some instances, the information included in the HHRA 
deviated from a particular section of the framework. For example, the HHRA may have included a criterion in the exposure assessment that should have been in the dose-response assessment. This was not considered a non-compliance providing the criterion was used in the right context and satisfied the best practice adequately. Table 5.1 below highlights how many criterion conformed to best practices and how many were conformed or deficient when compared to the framework.

The Dose-Response Assessment performed relatively well compared to the other stages within the HHRA. It did not omit criteria unlike the other stages, and presented a strong focus on vulnerable populations. The derivation of the TRVs was associated with a detailed explanation of how it was derived and also identified susceptible populations that were affected by different contaminants. This showed transparency on the risk assessors' part. Although many practices were conformed, the deficiencies are of greater importance for the purpose of this study. The dose-response stage did not adequately consider critical periods of development when deriving all the TRV levels. Different days of gestation were used to test one or two contaminants but this should have been conducted for all contaminants to determine accurate levels of TRVs for different populations, including fetuses. With organogenesis being a particularly critical period of growth for a fetus as mentioned in Chapter 2, it should have been considered in determining acceptable levels of emissions.

A minor deficiency was also present. For transparency purposes, it would have been beneficial for a separate discussion of uncertainties in the dose-response section. This would have allowed readers to better understand the limitations of the calculations and what it meant in terms of assessing risks. 
Expectations for the hazard identification, exposure assessment and risk characterization stages were met with a few deficiencies and absences. The major absence in the hazard identification stage was the omission of a distinct hazard identification step. As a result, data for this stage was dispersed throughout the risk assessment. Nevertheless, the health problems caused by each incinerator pollutant and the most susceptible members of the population were still identified. Literature and regulatory documents show that the hazard identification stage is beneficial in a comprehensive HHRA (United States Environmental Protection Agency, 2012b), therefore including this step would be valuable for readers since it provides greater transparency of data. Another deficiency was the discussion of delayed/late onset health effects. Although a discussion of delayed onset of health impacts was mentioned for one contaminant, this was not consistent for each contaminant and should have been, since many contaminants that were screened were carcinogenic, therefore produce cancerous effects later on in life. One can argue that the LADD (lifetime average daily dose) was calculated for the cancer causing contaminants, however, detail on each calculation was not provided, and the explanation of what the calculation meant for each contaminant was not provided either, but would have been beneficial. One criterion that was absent from this stage was a discussion of the strengths/limitation of data used to support the weight-of-evidence and other conclusions made in the section, but the omission of this stage in the HHRA could explain why this may not have been considered.

The risk characterization step also performed its task by providing risk estimates. Risks from carcinogens and non-carcinogens were calculated for the most sensitive receptors (infant and toddler) as well as for chemical mixtures. Uncertainties were also discussed in the section, 
but it was hoped that the discussion would include more about the uncertainty of TRVs and their protection of the most sensitive receptors.

\begin{tabular}{|l|l|l|l|}
\hline Stages of a HHRA & Conformities & Deficiencies & Absences \\
\hline Problem Formulation & $12 / 20$ & $7 / 20$ & $1 / 20$ \\
\hline Hazard Identification & $7 / 9$ & $1 / 9$ & $1 / 9^{*}$ \\
\hline Exposure Assessment & $10 / 13$ & $2 / 13$ & $1 / 13$ \\
\hline $\begin{array}{l}\text { Dose-Response } \\
\text { Assessment }\end{array}$ & $10 / 12$ & $2 / 12$ & 0 \\
\hline $\begin{array}{l}\text { Risk Characterization } \\
\text { Total: }\end{array}$ & $12 / 15$ & $2 / 15$ & $1 / 15$ \\
\hline
\end{tabular}

* There was a very important omission that would not be seen based on criteria, which was the omission of the entire hazard identification section from the HHRA. The criteria was scattered in other sections in the HHRA instead.

Table 5.1: The results of the Durham-York human health risk assessment when evaluated against the best practice framework

In addition, evaluating the risks specific to the general population and to the different vulnerable populations should have been done to put the risks in perspective, in addition to comparing the risks to another risk that can be easily understood by readers, as mentioned in section 4.5.2. Those who are concerned with projects such as this are usually members of the public, so in order to ensure that the risks are easily understood, the risks from the HHRA need to be compared to risks that are normally occurring in everyday life. Looking back at the doseresponse stage, the derivation of TRVs were expected to be safe for sensitive subjects following exposures (Ollson et al., 2014) but the risk characterization did not discuss whether the TRV was safe enough for vulnerable populations such as pregnant women and fetuses. Although it is likely the calculations for the TRVs considered these populations, mentioning it would further 
add to the clarity of the risk assessment. Another significant deficiency was regarding the exceedances of contaminant levels. Analysis of exposures showed that many contaminants exceeded the regulatory limits, particularly for toddlers and infants. Further confusion resulted with a weak justification as to why exceedances were present. Baseline levels were held accountable for the exceedances, but risk management measures were not identified to decrease these contaminant levels prior to the construction of the facility. The facility would further contribute to the atmospheric levels and this raises concerns of public health.

The exposure assessment also adequately conformed with best practices. However, the deficiencies proved to be significant. The Durham-York HHRA did not acknowledge a higher intake to body-weight-ratio for vulnerable receptors such as pregnant women and fetuses. Again, this was due to the fact that this sub-population was not considered originally in the assessment. Although toddlers were considered for this criterion, it was not clear why other receptors were not. Another deficiency identified in this stage concerned exposure factors used in the risk assessment. The exposure factors utilized did not clearly indicate whether they were adjusted for each life-stage. The absence of the trans-placental exposure pathway was also observed in this stage and was a result of the absence of pregnant women and fetuses in the problem formulation stage.

The problem formulation/screening section was undoubtedly the poorest in conforming to best practices. This stage had significantly more deficiencies than the rest. This stage sets the foundation for the entire risk assessment and it is important that these basic criteria are met before a risk assessment proceeds. Most of the general HHRA requirements were met, but became problematic at the inclusion of life-stage specific information. For instance, the 
problem formulation should have outlined the toxicity of the chemicals, and the organs that these chemicals affect. This was done in Appendix $\mathrm{H}$ but should have been linked to this stage as well since this is where the screening of contaminants generally takes place. The key deficiency was the exclusion of pregnant women and fetuses from the HHRA, with no indication that they are a vulnerable population worth considering. The risk assessor should not have limited the parameters at this stage and should have presented a greater variety of receptors before narrowing down which were more susceptible. The failure to provide a rationale for the exclusion of this population was another reason why this performed less adequately than the rest. Many of the weaknesses in this section stemmed from their exclusion.

Overall, out of a potential 69 best practice criteria, 51 criteria were strongly met in the health risk assessment indicating that the assessment reasonably conformed to best practices. However, it is important to analyze this further and isolate the general criteria from those that pertain directly to the consideration of vulnerable populations. Table 5.2 below shows the criteria from the framework that relate directly to vulnerable populations organized by those that conformed to best practices, those that were deficient and the number that were absent.

Evaluating the numbers shows that the criteria that related directly to vulnerable populations included $11 / 25$ of the criteria that conformed to best practices, $11 / 25$ that were deficient and $3 / 25$ criteria were absent. The numbers indicate that vulnerable populations in general were considered in the assessment but deficiencies were a result of the exclusion of pregnant women and fetuses and the lack of consideration for population susceptibilities and unique patterns of exposure. As a result, the vulenrabilities of pregnant women and fetuses were not explicitly considered in the HHRA. Considering "children" as a sequence of life-stages 
is the main adjustment that needs to be made in this HHRA and future conducted HHRAs. If this approach was taken on the Durham-York HHRA, it can be assumed that all the best practices would have been met and populations ranging from fetuses to the elderly and sick, would have been accurately protected.

\begin{tabular}{|l|l|l|l|l|}
\hline Stages of HHRA & $\begin{array}{l}\text { Total number of } \\
\text { criteria directly } \\
\text { relating to } \\
\text { vulnerable } \\
\text { populations }\end{array}$ & $\begin{array}{l}\text { Criteria that } \\
\text { conformed to best } \\
\text { practices }\end{array}$ & $\begin{array}{l}\text { Deficient best } \\
\text { practice criteria }\end{array}$ & $\begin{array}{l}\text { Absences of } \\
\text { best } \\
\text { practice } \\
\text { criteria }\end{array}$ \\
\hline $\begin{array}{l}\text { Problem } \\
\text { Formulation }\end{array}$ & 8 & $1 / 8$ & $6 / 8$ & $1 / 8$ \\
\hline $\begin{array}{l}\text { Hazard } \\
\text { Identification }\end{array}$ & 4 & $3 / 4$ & $1 / 4$ & $0 / 4$ \\
\hline $\begin{array}{l}\text { Exposure } \\
\text { Assessment }\end{array}$ & 6 & $3 / 6$ & $2 / 6$ & $1 / 6$ \\
\hline $\begin{array}{l}\text { Dose-Response } \\
\text { Assessment }\end{array}$ & 5 & $4 / 5$ & $1 / 5$ & $0 / 5$ \\
\hline $\begin{array}{l}\text { Risk } \\
\text { Characterization }\end{array}$ & 2 & $0 / 2$ & $11 / 25$ & $1 / 2$ \\
\hline \multicolumn{1}{c}{ Total: } & 25 & $1 / 25$ & $3 / 25$ \\
\hline
\end{tabular}

Table 5.2: The conformities, deficiencies and absences of criteria in the best practice framework by the Durham-York human health risk assessment that pertained directly to vulnerable populations

\subsection{Summary of Research Methods and Major Findings}

\subsubsection{Summary of Research Methods}

The four research objectives for this study were presented in the introductory sections of this thesis. The first research objective was to establish HHRA best practices for incineration projects from authoritative sources on HHRA primarily for vulnerable populations (i.e. pregnant women and fetuses). A comprehensive literature review, evaluation of previously conducted HHRAs, and interviews done from authoritative experts was done in order to identify these best practices. The interview process proved difficult since recruiting participants was not as easy as 
initially assumed. Out of the 71 experts contacted via email invitations, a total of seven people agreed to participate and complete the interview process.

The second research objective was to create a framework to organize the best practices in the form of a checklist that was divided up into the five sections (problem formulation, hazard identification, exposure assessment, dose-response assessment and risk characterization) of an HHRA. This made it easier to go through the list and to compare it with a HHRA to see whether criteria were met.

The third research objective was to compare and apply the best practices framework to the Durham-York HHRA. This was conducted by section but the best practices were not considered necessarily in the same order in the HHRA as listed in the framework. Wherever a best practice was found in the HHRA, it was checked off on the framework. Wherever a best practice was not identified, the letter " $\mathrm{N}$ " was marked next to the criterion, indicating it was not present.

The fourth and final research objective was to determine if the HHRA followed best practices and to the extent it followed them, which was the main focus of the HHRA. If a best practice was identified in the risk assessment, a thorough evaluation was completed to determine if the criterion conformed to best practices or was deficient. The conformities, deficiencies and absences were totaled for each section to help determine how well the Durham-York HHRA conformed to best practices and a separate analysis showed how well vulnerable populations were considered. 


\subsubsection{Summary of Major Findings}

Overall, it was apparent that the Durham-York HHRA did not explicitly state that pregnant women and fetuses were among members of the vulnerable population identified. This was the main reason why there were deficiencies and absences present in the evaluation of the best practice criteria. Although this specific population was not identified as a vulnerable population, toddlers and infants were. For this reason, it is reasonable to assume that the HHRA offered sufficient analysis for judging the risks of a normal healthy populations as well as for younger life-stages such as infants and toddlers. Those who had pre-existing health conditions - particularly respiratory conditions-were not listed as a vulnerable population of concern in the HHRA, although it would be reasonable to assume they should have been. Although there was no direct identification of pregnant women and fetuses as a vulnerable population, they were identified as susceptible to numerous contaminants that were identified in the risk assessment. However, it was unclear whether they were implicitly considered in the calculation of all TRVs chosen. It was unclear why this sub-population was considered highly susceptible to most of the contaminants identified but not reflected in the risk assessment.

Due to the implicit considerations, this study found that vulnerable populations were considered in the Durham-York HHRA and conformed to best practices, with some notable deficiencies presented earlier. For future HHRAs conducted for incinerators and other pollution emitting facilities, it would be reasonable and recommended to identify pregnant women and fetuses as a separate vulnerable sub-population rather than just implicitly considering them in the assessment. This conclusion was also made by a participant when asked the final openended question at the end of the interview process. They stated that "the [HHRA] should 
describe how it has included consideration of in utero into the estimate of risk" (Interviewee C, per. comm., 2014). Overall, it is clear that age-related exposures are an important consideration to determine the susceptibility of an individual and the Durham-York HHRA did not take into account the vulnerabilities of pregnant women and fetuses separately from other highly exposed individuals. Although a majority of the assessment conformed to best practices, the deficiencies that were outlined were essential to meet in order to confirm that the DurhamYork HHRA fully considered pregnant women and fetuses. However, many deficiencies were minor compared to other best practice criteria, and if the HHRA took a life-stage approach when conducting the risk assessment, these deficiencies would not have existed.

\subsection{Study Limitations}

There were a few study limitations that were apparent in this research and important to note. One limitation emerged due to the low number of participants that were interviewed for the research. Although seven interviewees are a viable number of research participants for analysis purposes (Miller, 2012), a broader sample size of interviews was anticipated to generate a greater level of confidence in the research and the framework. However, due to conflicts of interests and busy schedules for many experts, seven participants was the maximum number recruited to take part in the study. Further limitations arise from the low recruitment number such as the adequacy of this sample size and whether it encompasses the views of a greater number of experts. 
Another limitation was the difficulty in finding research the pertained to vulnerable populations and health risk assessments. When conducting the literature review, although a vast number of articles were found regarding health risk assessments, many of them lacked detail and focused only on certain aspects of risk assessment. More specifically, vulnerable populations and the deficiencies in health risk assessments proved to be a gap in the literature since very few articles were found supporting the issues with risk assessments.

Some of the limitations overlapped with Mueller's (2010) study limitations, due to a similar method used for analysis. These included whether enough research was done to support the criteria listed in the framework, and whether the use of the literature and using experts to support the best practices, was appropriate. This process was a two-step method, because it was understood that simply using the literature would not include primary information from experts who have up-to date knowledge and experience with these matters. Both methods were put in place to ensure that there was a degree of support and confidence when adding a criterion to the framework. Mueller (2010) had also stated that a limitation was a result of the Principal Investigator being the only individual conducting the literature review and interviews and extracting the information from both to create the best practice framework. In addition, the Principal Investigator was the only individual comparing the HHRA to the framework and assessing whether the HHRA was performed according to best practices. This limitation was unavoidable due to the nature of the study, meaning the identity of the participants was to remain anonymous to everyone except the Principal Investigator. Ethically, any other individual reviewing the contents of the interviews to extract best practices would have been inappropriate. Therefore, as Mueller (2010) stated, this limitation was unavoidable. 
Each study was reviewed multiple times to make certain that a criterion listed as a best practice, was in fact one.

\subsection{Potential Application of the Research}

This framework may assist in environmental science and management fields for future incineration HHRA reviews in Ontario. This research will be relevant under the EAA for incinerator HHRAs as it can be used as an evaluation method. This may be used by the proponent/consultant when drawing up an HHRA, and can be used by the government when they review the HHRA for approval. This will allow the proponent to save time when drawing up an HHRA and ensuring that all criteria are present. It may also assist the government by speeding up their review process with a checklist and help ensure that everything needed to protect pregnant women and fetuses, is present.

In addition, this research can be used as an evaluation model for the new Peel Region Energy Recovery Centre HHRA which was approved in June 2013 (Peel Energy Recovery, 2015). This facility is still in its initial stages of planning, undergoing public consultations at the moment. Planning and approvals are expected to be completed by 2017, when design and construction is expected to begin (Peel Energy Recovery, 2015). The facility is to start by 2020 for an operational period of 30 years or more (Peel Energy Recovery, 2015). It will be interesting to see if the results found in this research are similar or different in terms of strengths and weaknesses of the HHRA compared to the Durham-York analysis. Furthermore, 
the findings of this study can ultimately be used for strengthening HHRAs for other polluting facilities as well and ensure that the populations are effectively being protected.

\subsection{Future Research Initiatives}

There are several future research initiatives that this thesis may contribute to when completed. It was previously stated that there were limitations in finding the data and literature for this framework, therefore further research and consultation with experts may produce a more extensive framework. The inclusion of vulnerable populations, particularly pregnant women and fetuses is currently where a gap exists in the literature. Therefore, further research in this area in future years can contribute valuable input into this framework.

Another research question that can be asked from this current Durham-York HHRA is whether the uncertainty factors used were truly accurate in accounting for the intraspecies variation between the different life-stages. Pelekis, Nicolich and Gauthier (2003) conducted research on this topic independent to this HHRA as this is still a growing niche that would be beneficial to risk assessment research to better protect all life-stages. Landrigan et al. (2004) stated that the 10x uncertainty factor was appropriate for infants, but may not be sufficient to account for unique susceptibilities of fetuses. Felter et al. (2015) have recently conducted research on the use of uncertainty factors, particularly for susceptible life-stages. They also stated that the use of these factors were appropriate for all populations particularly infants, but did not identify if the fetal life stage was among the group that was protected as well. It would be interesting to see research focusing solely on uncertainty factors for fetuses. 


\subsection{Summary and Conclusion}

Overall, this chapter has provided a discussion of the results, summary of the research methods, followed by the major findings of this research. The limitations associated with the research were identified as well as the potential contribution of this type of research. Finally, the chapter ends with a discussion of potential future research initiatives and how the research conducted here can be used for further research needs and ideas.

To conclude, HHRAs are critical for the protection of public health from polluting facilities such as incinerators. Questions were initially raised whether the Durham-York HHRA complied with best practices and whether it adequately considered pregnant women and fetuses. However, analysis showed that most of the HHRA complied with best practices but the explicit consideration of pregnant women and fetuses was neglected/omitted. Therefore, the risk assessment protects the robust healthy non-vulnerable population but the study reveals that there is an inadequate assessment of vulnerable populations. With interest growing in vulnerable populations and their inclusion in risk assessment, the best practice framework presented in this thesis hopes to create stronger risk assessments in the future, forcing them to cover details that are not commonly included, but should be. Overall, it is anticipated that this thesis will fill a gap in the literature pertaining to pregnant women and fetuses and their inclusion in HHRAs. The framework can be utilized as a guideline to assess future HHRAs as well. 


\section{Appendix A: Research Consent Form}

\section{RYERSON \\ UNIVERSITY}

CONSENT TO PARTICIPATE IN RESEARCH

A Critical Analysis of Vulnerable Populations in the Durham-York Energy-From-Waste Incinerator Health Risk Assessment

You are being invited to participate in a research study. Please read this Consent Form so that you understand what your participation will involve. Before you consent to participate, please ask any questions necessary to be sure you understand what your participation will involve.

\section{INVESTIGATORS}

This research study is being conducted by the Principal Investigator Nazira Panchbhaya with faculty supervisor Dr. Ron Pushchak from the Environmental Applied Science and Management Department at Ryerson University. The results from this research will contribute to the Principal Investigator's thesis project.

If you have any questions or concerns about the research, please feel free to contact:

Principal Investigator- Nazira Panchbhaya

Email: nazira.panchbhaya@ryerson.ca

Faculty Supervisor- Dr. Ron Pushchak

Email: pushchak@ryerson.ca

Phone Number: 416-979-5000 x 7049

\section{PURPOSE OF THE STUDY}

Best practices in a Health Risk Assessment are methods accepted for incineration projects, those methods that are common amongst experts and the literature, and are deemed acceptable, effective and protect human health.

This study will examine the Health Risk Assessment and the peer review of the Durham-York mass burn incinerator. The goal is to determine whether human health was sufficiently protected, specifically focusing on vulnerable populations to see whether best practices were followed. 


\section{DESCRIPTION OF THE STUDY AND YOUR PARTICIPATION}

If you volunteer to participate in this study, you will be asked to do the following things:

\section{Accepting/Declining the Participation Offer: Providing Consent}

The Principal Investigator will send you an email with an interview package which will contain the consent form, interview template as well as information about the research. As a selected participant, you will be asked to review the material including interview questions prior to signing any consent. After reviewing all the information and questions provided, you may either choose to accept or decline the offer to participate in the research.

If you choose to participate, you will be asked to read the consent form and send an email to the Principal Investigator indicating that you have read the consent form and that you agree to participate. You will also have to decide the method you would like to use to answer the questions, either by telephone or via email in the form of written answers (full paragraph are not required). If you choose the telephone method, you have the option of having it recorded, if comfortable. If not, you will not be pressured to do so. If you would like to choose the telephone method AND be recorded, you must also consent to the telephone recording section of the consent form, in the same method mentioned above. If you would like to choose the telephone method but not be recorded, this portion of the consent form does not have to be completed, but whether choosing the email or the telephone method, either has to be indicted in the consent email you send to the Principal Investigator. In addition, you are welcome to review or edit any recordings or transcriptions from the interviews (telephone/email). If you choose to decline participation in this study, no further action will be required from your part and you may ignore the emails.

As stated above, if you choose to participate, you must read the consent form and send an email to the Principal Investigator indicating that you have read the consent and are agreeing to participate. The email address is nazira.panchbhaya@ryerson.ca. Other methods of submission can be arranged with you and the Principal Investigator, if necessary.

\section{After you Provide Consent}

After the consent process, you may begin completing the interview questions via email. If you chose to participate by telephone, the Principal Investigator will contact you to schedule an interview.

When answering the interview questions, you may feel free to skip questions that you do not want to answer. You will not be asked why you chose not to answer the specific question. Completion of the questions should take about 45-60 minutes. If answering the questions by email, full paragraphs are not required and detailed jot notes are permitted.

Questions will be open-ended and will be chosen specifically to assist the researcher in determining best practices for Health Risk Assessments for incineration projects. An example of a question is:

\section{What is the most prominent authority from which to base HHRA standards from?}

Once the questions are completed to the best of your ability, you will be required to submit the interview questions via email to nazira.panchbhaya@ryerson.ca, unless other arrangements of submission are made personally between you and the Principal Investigator. If you have chosen to answer the questions by telephone, the Principal Investigator will be collecting the information during the telephone interview, therefore you are not required to re-submit your responses to the questions. 


\section{Submitting the Draft List of Best Practices}

After all of the responses to the interview template are received, the Principal Investigator will analyze the data and create the Health Risk Assessment best practices framework for incineration projects. Once completed, you will be invited to review the draft best practices framework. This will be submitted to you and the rest of the expert participants (optional) for your analysis and comments on whether the framework incorporates the correct practices for Health Risk Assessments specifically for incineration. Additional questions will not be provided as this step is more of a "check" to see if important best practices are incorporated. If you feel at this point that additional information is required in the framework, you may make corrections, additions or deletions directly onto the provided framework. If you feel that your responses were interpreted inadequately, then you may make this comment on the framework provided of what you felt was incorrect. Once you submit this information, the Principal Investigator will look into the additional revisions and compare them to the responses of other participants. If there is a unanimous agreement to a specific addition, deletion or correction of information, the Principal Investigator will make these changes to the framework. If there is a misinterpretation of your responses specifically, this will be addressed by the Principal Investigator and the changes will be reflected in the final copy upon your approval. However, you must remember that this framework is a best practices framework, meaning that each step in the framework must be common amongst the literature, other Health Risk Assessments and experts like yourself. If you feel that something is a best practice but this is not common amongst all sources, it may not be included in the framework. After this check is completed by you, your participation in this study are complete.

Your identity will be confidential throughout the entire process. The only identifier that will be used in the thesis and any following publications is your job title, whether it be a public health practitioner, risk assessor etc. To maintain further confidentiality, you will be assigned a random number (i.e. interviewee \#1, 2, 3 etc.) that will be used to report data in any published material. You may choose not to partake in the review of the best practice list as this section is optional, but your interview responses will still be used in the study. If you choose to withdraw all of your information at this point of the study, you may do so with no questions asked.

\section{POTENTIAL RISKS AND DISCOMFORTS}

The potential risks and discomforts for taking part in this study are very low. One minimal risk/discomfort is that your job title will be mentioned in this study. In addition, if you choose to complete the interview by telephone, the Principal Investigator will hear your voice during the interview process. As mentioned, these are all minimal to no risk events, but will be mentioned for your understanding.

It is important to remember that your identity will remain completely confidential. Although the job title will be mentioned, it will not be a specific job title. Instead, a general title such as "public health practitioner" will be used. There is no way of linking the job title to your identity since the participants are being selected from different institutions in different countries and no other identifiers will be provided.

The interview questions are non-invasive, open-ended questions so that you can comfortably answer the questions the best to your knowledge and understanding. The questions are not designed to cause any sort of discomfort, but if they do, you can feel free to skip any questions. You will be given a reasonable time period to answer and submit the completed interview template either via email, or telephone interview. You may also choose to discontinue participating for any reason at any time during the interview process.

In addition, two methods of interview are provided in this study. This has been done so you are completely comfortable answering the interview questions. If you feel uncomfortable allowing the Principal Investigator to hear your voice during the telephone interview, you may easily choose the email interview method instead and provide written responses to each question to minimize any discomfort you may feel. 
All precautions have been taken to ensure that full confidentiality is maintained in this study, but like in every study, there is always a slim chance that identity is revealed. This risk has been minimized in the sense that all the questions are not invasive and will not compromise your occupation level in any way if identity is revealed in any way. Furthermore, primary data sources with any identifiers that was used by the Principal Investigator during the study will be destroyed after the approval of the study.

The study is to simply capture what best practices for incineration include, therefore you are only providing expert knowledge in your field, and therefore the risk is very minimal.

\section{POTENTIAL BENEFITS TO PARTICIPANTS AND/OR TO SOCIETY}

The information that will be gathered in this study can potentially benefit government regulators, consultants as well as proponents for incineration projects by providing evaluation criteria for incineration Health Risk Assessments prior approval and it can assist the government by speeding up their review process.

Although potential benefits are mentioned, we cannot guarantee any benefits for your participants.

\section{PAYMENT FOR PARTICIPATION}

You will not be paid to participate in this study.

\section{CONFIDENTIALITY}

Confidentiality will be maintained throughout the study to the extent that names, gender, age, or any institution that you are affiliated with, is not mentioned. The only identifier that will be mentioned in the study is your occupation, whether a public health practitioner, risk assessor or other. All identifiers in this thesis will be removed for publication, except the job field. Your identity will not be or revealed in this study. As mentioned previously, you will be assigned a random number (Interviewee \#1, 2,3) that will be used by the Principal Investigator for organizing the data and for publishing purposes. For example, it will say that "interviewee\#1 stated..." The job title will be mentioned for means of showing your credibility along with the rest of the participants.

As mentioned, if you choose the telephone interview method and allow it to be recorded, you have the right to review and edit any recordings or any transcripts that were made from the interview by simply requesting the Principal Investigator to send these to you. If you choose the email interview option, you may change your written responses even after submitting it to the Principal Investigator. This will not be reflected or mentioned in the study. However, once the thesis project has been completed and submitted for approval, you will not be able to make changes to your responses since the data will have been analyzed at this point.

In regards to the information collected during the study, the Principal Investigator will be the only individual to have complete access to the data to maintain your confidentiality. The supervisor Dr. Ron Pushchak may also have access to data if necessary to help in the analysis of data collected. The data will be stored on a personal computer, where only the Principal Investigator will have access to with a secured database. Once the thesis has been approved by the board, the primary data (your original written responses via email as well as telephone recordings and transcripts) will be transcribed into a personal database without any identifiers of the individual. The original emails and telephone recordings with all identifiers will be destroyed after a one year time period. 


\title{
VOLUNTARY PARTICIPATION AND WITHDRAWAL
}

Participation in this study is voluntary. You can choose whether to be in this study or not. If you volunteer to be in this study, you may withdraw at any time without consequences of any kind. If you choose to withdraw from this study you may also choose to withdraw your data from the study. You may also choose not to answer any question(s) and still remain in the study. Your choice of whether or not to participate will not influence your future relations with Ryerson University.

\section{QUESTIONS ABOUT THE STUDY}

If you have any questions about the research now, please ask. If you have questions later about the research, you may contact:

\author{
Nazira Panchbhaya \\ Principal Investigator \\ nazira.panchbhaya@ryerson.ca \\ Ron Pushchak \\ Faculty Supervisor \\ pushchak@ryerson.ca \\ 416-979-5000 x 7049
}

This study has been reviewed by the Ryerson University Research Ethics Board. If you have questions regarding your rights as a research participant in this study, please contact:

Toni Fletcher, Research Ethics Coordinator

Research Ethics Board

Office of the Vice President, Research and Innovation

Ryerson University

350 Victoria Street

Toronto, Ontario M5B 2K3

416-979-5042 or toni.fletcher@ryerson.ca 


\section{SIGNATURE OF RESEARCH PARTICIPANT/EMAIL WITH CONSENT}

Your signature below indicates that you have read the information in this agreement and have had a chance to ask any questions you have about the study A Critical Analysis of Vulnerable Populations in the Durham-York Energy-From-Waste Incinerator Health Risk Assessment as described herein. Your questions have been answered to your satisfaction, and you agree to participate in this study. You have been given a copy of this form. For your convenience, you may read the consent and send the Principal Investigator an email indicating that you have read the consent, and agreeing to participate in the study as well as the method you would like to use to be interviewed.

Name of Participant (please print)

Signature of Participant

Date

\section{CONSENT FOR AUDIO RECORDING (VOLUNTARY- PARTICIPANT CAN CHOOSE NOT TO USE THIS METHOD OF INTERVIEW OR NOT BE RECORDED IF THEY DO CHOOSE THIS METHOD)}

This study allows you to participate via two methods. One is an email interview where you can write out the responses to the questions and submit it to the researcher. The second is a telephone interview method where the researcher will schedule an interview time with you. Before the telephone interview is conducted, the researcher will ask whether you are comfortable with an audio recording of the interview. If you agree, the researcher will record the interview and you will have the ability to review and edit the recordings and transcripts upon request. If you do not agree to be audio recorded, the researcher will not pressure you in any way to do so and the interview will commence.

A signature is required below IF you choose the telephone interview method opposed to the written email method, AND you agree to be audio recorded. If not, the telephone interview method can still be used without an audio recording, but the researcher will take notes during the interview for research purposes. If choosing the interview method but preferring not to be audio recorded, the section below does not have to be filled out. For your convenience, you may read the consent and send the Principal Investigator an email indicating that you have read this section of consent, and are agreeing to be audio recorded.

Name of Participant (please print) 


\section{Appendix B: Letter of Invitation}

\section{RYERSON}

UNIVERSITY

Title of Study: A Critical Analysis of Vulnerable Populations in the Durham-York Energy-FromWaste Incinerator Health Risk Assessment

Principal Investigator: Nazira Panchbhaya, Graduate Student, Masters of Environmental Applied Science and Management, Ryerson University

Faculty Supervisor: Dr. Ron Pushchak, Department of Occupational and Public Health, Ryerson University

Dear Participant,

I, Nazira Panchbhaya, a graduate student in the Masters of Environmental Applied Science and Management Program at Ryerson University invite you to participate in a research project entitled: A Critical Analysis of Vulnerable Populations in the Durham-York Energy-From-Waste Incinerator Health Risk Assessment.

The purpose of this research project is to examine the Health Risk Assessment and the peer review of the Health Risk Assessment for the Durham-York mass burn incinerator. The goal is to determine whether human health was sufficiently protected, specifically focusing on the vulnerable population of pregnant women and fetuses to see whether Health Risk Assessment best practices were followed. The literature and expert knowledge will be used to determine and create a Health Risk Assessment best practices framework.

Should you choose to participate, you will first be asked to read the consent form and send an email to nazira.panchbhaya@ryerson.ca that you have read the consent and agree to participate. This process is for your convenience as it will eliminate the process of printing the consent form, signing it and then scanning it back to me via email. It is important to remember that when you send the consent email back to the Principal Investigator, you will have to indicate which method of interview you would like to choose, whether the telephone method or the email interview method. A simple statement indicating this such as "I would like to choose the email/telephone method for interview purposes" in the consent email to the Principal Investigator will suffice. The interview template is also attached to this email so you can look over the types of questions you will be asked to complete before signing the consent form. If you have further questions about the study prior to deciding whether you will participate or not, please feel free to contact the Principal Investigator via email at nazira.panchbhaya@ryerson.ca. 
The Principal Investigator will contact you via email a week from the original email as a friendly reminder to read and complete the consent process if you would like to participate in the study, if you have not already done so. If not, you can ignore the emails and you will not be contacted again in regards to this study.

If participating and choosing the telephone interview method AND allowing the Principal Investigator to audio record the interview, then the audio recording section of the consent should be read and consent for this section should be given separately in the consent email, therefore, a total of two consents. One is for participating in the study and the other is for the audio recording, if you choose this option. If you choose to use the telephone interview method and would not like to be audio recorded, then your consent email only needs one consent, which is for the entire study. A simple statement could be stated in the email indicating that you would like to participate. The interview will take about 45-60 minutes to complete. Full paragraphs are not required to answer the questions and jot notes are permitted.

Once the interview questions are completed, the answers will be collected by the Principal Investigator and will be used to create a Health Risk Assessment best practices framework. Once this framework is completed, the Principal Investigator will invite participants via email to look over the draft framework to see whether they feel it accurately includes best practices for incineration Health Risk Assessments. As an expert participant, you may comment on the framework and analyze to see whether it captures best practices. Once this is done, you will be asked to send the comments, if any, to the Principal Investigator, so changes can be made, if required.

This research can potentially benefit government regulators, consultants as well as proponents for incineration projects by providing an evaluation criteria for incineration Health Risk Assessments prior approval and it can assist the government by speeding up their review process.

Confidentiality will be maintained throughout the study to the extent that names, gender, age, or any institution that you are affiliated with, is not mentioned. The only identifier that will be mentioned in the study is your occupation, whether a public health practitioner, risk assessor or other. All identifiers in this thesis will be removed for publication, except the job field. Your identity will not be revealed in this study. You will be assigned a random number (Interviewee \#1, 2, 3) that will be used by the Principal Investigator for organizing the data and for publishing purposes. For example, it will say that "interviewee\#1 stated...". The job title will be mentioned for means of showing your credibility along with the rest of the participants. 
This study has been reviewed by the Ryerson University Research Ethics Board. If you have questions regarding your rights as a research participant in this study, please contact:

\author{
Toni Fletcher, Research Ethics Coordinator \\ Research Ethics Board \\ Office of the Vice President, Research and Innovation \\ Ryerson University \\ 350 Victoria Street \\ Toronto, Ontario M5B 2K3 \\ 416-979-5042 or toni.fletcher@ryerson.ca
}

If you have any other questions regarding the content of the study itself, please feel free to contact me (see below for contact information).

Thank you,

Nazira Panchbhaya

Principal Investigator

nazira.panchbhaya@ryerson.ca

Dr. Ron Pushchak

Faculty Supervisor

$416-979-5000 \times 7049$

pushchak@ryerson.ca 


\section{Appendix C: Interview Template}

\section{Interview Questions}

\section{All of the following questions relate to municipal solid waste incinerator Human Health Risk Assessments (HHRA)}

1. How should vulnerable populations be defined in a health risk assessment?

2. What are the necessary parameters that should be considered when identifying vulnerable populations?

3. Should sensitive/vulnerable populations such as the fetus (those of pregnant women) be assessed for a health risk assessment separately/differently than the normal population?

4. What are potential health hazards from start-up and shut-down conditions for incinerators? ii.) Should these scenarios be included in a health risk assessment for incinerators?

5. Which priority health issues of new incinerator facilities should be given special attention when constructing such facilities?

6. Are standards used for air contaminants released from incinerators appropriate in considering different components of the population?

7. What are all the exposure pathways to be considered when assessing the health risks for fetuses?

8. How should the exposure assessment differ for vulnerable populations such as pregnant women and fetuses from other populations? (Do you feel additional data is required for this population?)

9. It is understood that 1 in a million is an appropriate carcinogenic risk standard for general populations. a.) Is this standard appropriate for fetuses? b.)If not, which risk standard is appropriate and should be specifically used for fetuses?

10. Identify and discuss what the most prominent authority is to base HHRA standards?

11. Should dioxins and furans, mercury and arsenic be assessed differently in a HHRA for robust populations and vulnerable populations? (Should the standard be more stringent?) 
12. Should baseline information be considered in an HHRA? ii.) What is the best method of identifying baseline information needed to undertake effective monitoring for the health of pregnant women and fetuses?

13. Discuss the use of precedents for exposure from previous HHRAs. Do you feel these are relevant and appropriate?

14. Should the risk characterization specifically describe risks to sensitive/vulnerable populations?

15. Should it be a best practice to report the uncertainty of exposure/chemical uptake in an environment in an HHRA?

16. How important is it to include a discussion of uncertainties in the risk characterization stage? What should this discussion include?

17. How important is it to use the precautionary approach in health risk assessments for incinerators and why?

18. What should the publics' role in HHRA be? ii.) Which stages should there be involvement?

19. Is there additional information that should be available to the public from HHRAs?

20. Discuss any other information that should be included in the HHRA specifically for the fetus of pregnant women or any best practices that should be included? 


\title{
Appendix D: Human Health Risk Assessment Best Practice Framework with Emphasis
}

\section{on Vulnerable Populations}

\author{
Human Health Risk Assessment Framework for Incineration Facilities: Focus on Whether \\ Vulnerable Populations (Pregnant Women and Fetuses) are Adequately Protected
}

The framework below is set up as a checklist, meaning that each criteria requires a "yes" or "no" response indicating whether the best practice is present in the HHRA. The strengths and weaknesses of the "yes" responses can be determined by the evaluator, who can ultimately decide if additional information is needed to satisfy the criteria. If a "no" is indicated beside a criteria, this means that the criteria was not included and the evaluator can decide if it should have been, depending on the circumstances.

\subsection{Problem Formulation/Screening Best Practice Criteria}

1.1 The risk assessment uses a quantitative assessment of risk 351233354463

1.2 Identifies the geographical location of the facility 29121922313359

1.3 Identifies land use 1011121318222329335559

1.4 Identifies the baseline environmental conditions 5101636394041435563

1.5 Identifies the air emission control technology to be used 12131922293059

1.6 Identifies chemicals of concern that will be included in the risk assessment (prominently cadmium, mercury, arsenic, PAHs, PCBs, dioxin and furans) 25791112151722262728313344555663

1.6.1 Chemicals also include criteria air pollutants 51226

1.6.2 Determines the toxicity of the chemicals specific to life-stages (sensitivity of receptors to chemicals) 34263

1.6.3 Determines the organs/developmental systems that these chemicals may affect or if they cause any critical organ-specific toxicity 317424647

1.7 Identifies the preliminary receptors/potentially highly exposed life-stages (i.e. pregnant women) that are the most sensitive to the chemicals of concern identified and those more likely to be exposed 235334245475163

1.7.1 Defines vulnerable or highly exposed populations/life-stages using numerous parameters (i.e. age, body weight, fetuses, pregnant women, racialized communities, those with pre-existing health conditions etc.) ${ }^{13} 1116192133353940414247545563$

1.7.2 Provides a rationale for focusing on the particular groups of receptors/life-stages or why certain age groups experience higher exposures or are more susceptible 3465863

1.7.2.1 Specifically identifies fetuses/pregnant women as a vulnerable group? ${ }^{13111621}$ 3940414243475463

1.7.3 Detailed justification of any receptor/age group excluded provided 33354663

1.8 Identifies the most likely route of uptake of chemicals for different life-stages/receptors (i.e. gastrointestinal absorption, dermal penetration, respiratory tract absorption/inhalation, ingestion trans-placental or a combination) 34651

1.9 Outlines a conceptual model to guide the risk assessment to consider sources, receptors (lifestage groups), stressors, exposure pathways/scenarios and individual characteristics of chemicals and endpoints (i.e. toxicity to organs) 1357243133354245475051556163

1.9.1 Provides justification for any omitted/incomplete routes or pathways 123335

1.10 Allows for public/stakeholder input 15716394041424350

1.10.1 Transparency of information is present 516424350

1.11 Discusses the use of any precedents (from other HHRAs) for data purposes 1612164142 
1.1

1.2

1.3

1.4

1.5

1.6

1.6.1
1.6 .2

1.6.3

1.7

1.7.1

1.7 .2

1.7.2.1

1.7.3
1.8

1.9

1.9.1

1.10

1.10 .1

1.11

Summarize the compliances, deficiencies and absences present in this section and determine whether the problem formulation/screening stage was completed sufficiently. 


\subsection{Hazard Identification Stage Best Practice Criteria}

2.1 Identifies animal-based and/or available human-based toxicology studies to determine the type and nature of health effects from contaminants of concern 42444750586164

2.1.1 Accounts for whether effects from animal studies are different than what humans will ultimately experience when extrapolating data from animals to humans and /or when extrapolating data from human adults to earlier life-stages 4447506364

2.1.2 Determines weight-of-evidence to see whether chemicals may pose a carcinogenic risk ${ }^{1}$ 6123542445058

2.2 Provides characterization of hazardous/toxic effects or endpoints for each selected chemical (adverse effects/outcomes) 579121731354244475056

2.2.1 Identifies under which conditions the hazard can be expressed ${ }^{1173456}$

2.2.2 Determines the life-stage-specific health effects/health outcomes (specifically for pregnant women where necessary) 342474850

2.2.3 Considers the potential for harm during critical windows of development and/or also considers delayed effects/late onset of chemical effects 5424748515358

2.2.4 Determines the most susceptible life-stages for exposure (i.e. pregnant women [fetuses] breast feeding infants) 42445051535863

2.3 States the strengths and /or limitations of data used to support the weight-of-evidence and other conclusions made in this step including data gaps and uncertainties ${ }^{1424750}$
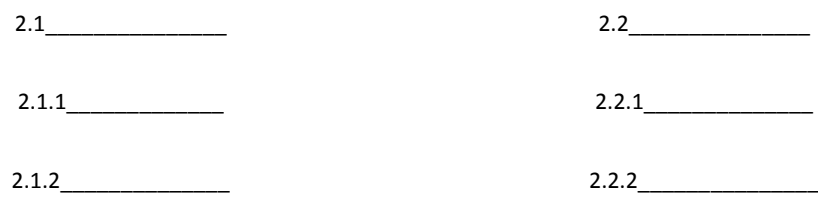

2.2 .3

2.2.4

2.3

Summarize the compliances, deficiencies and absences present in this section and determine whether the hazard identification stage was completed sufficiently. 


\subsection{Exposure Assessment Best Practice Criteria}

3.1 Identifies and characterizes all receptors including vulnerable receptors and other highly exposed groups 23571115182225313335545961

3.1.1 Considers breathing rates, patterns of food consumption and other physiological changes for vulnerable populations 1646474860

3.1.2 Identifies and evaluates receptor locations 51115223155

3.1.3 Acknowledges a higher intake rate to body weight ratio for fetus/children 431444760 3.2 Identifies various pathways and routes of exposure 1311121731323547516163

3.2.1 Includes specific pathways of exposure to the fetus/young children via ingestion of breast milk? 1112151934414751 or trans-placental 3424647485160

3.3 Uses exposure factors that are adjusted for relevant life-stages of concern (i.e. fetus), if necessary 42434751

3.4 Provides the concentration of the pollutant in the exposure media 35122225273347505256

3.4.1 Identifies the air dispersion model (i.e. CALPUFF, AERMOD) 12589111213222325303233444754 57

3.4.2 Identifies the results of deposition modelling 12252932

3.5 Presents a discussion of start-up/shutdown/accidental conditions 1216213839404155

3.6 Discussion of strengths/ limitation/assumptions of the data and the models presented ${ }^{133355143}$

3.1

3.1 .1

3.1 .2

3.1.3
3.2

3.2.1

3.3

3.4
3.4 .1

3.4.2

3.5

3.6

Summarize the compliances, deficiencies and absences present in this section and determine whether the exposure assessment stage was completed sufficiently. 


\subsection{Dose-Response Best Practice Criteria}

4.1 Identifies the maximum dose humans can receive without any adverse effects or the relationship between the dose and the severity of the effect 1257152227282931323435444763

4.2 Identifies susceptibilities of different life-stages for different biological/organ systems when exposed to chemicals (immune, nervous, respiratory, reproductive effects) 3432474858

4.2.1 Identifies critical periods of fetal development and organogenesis to help identify doseresponse relationships associated with chemicals 3474858

4.3 Identifies toxicity reference values (RfD, CSF, RfC, SF, URF, TDI, ADI) 1243335566163

4.3.1 Dose-response data obtained for at least one of the following reliable sources: obtained from at least one of the following reliable sources: MOE, Health Canada, USEPA, WHO,

California EPA, ATSDR 25162122313335394041434456616263

4.3.2 Rationale provided of all exposure limits/ toxicity reference values used 5313235

4.4 Identifies carcinogenic and non-carcinogenic endpoints for each chemical agent and for the different types of exposures 313233

4.4.1 Identifies different health effects associated with the exposure to a chemical agent (i.e. cancer, mortality, developmental delays, birth defects, low birth weight) ${ }^{42} 4748$

4.5 Identifies chemical-specific and/or population/life-stage specific factors which includes in effects on pharmacokinetics (absorption, distribution, metabolism, and/or excretion) 4245464764

4.6 Uses adjustment/uncertainty factors to account for specific receptors (i.e. fetal life-stage) 14224244 475864

4.7 Evaluates possible chemical interactions of one or more of the following: additivity, antagonism, synergism, potentiation 51218233335

4.8 Discusses significant uncertainties in the dose-response step ${ }^{122} 32344447$

4.1

4.2

4.2 .1

4.3

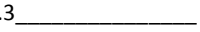

4.3.1

4.3.2

4.4

4.4 .1
4.5

4.6

4.7

4.8

Summarize the compliances, deficiencies and absences present in this section and determine whether the dose-response assessment stage was completed sufficiently. 


\subsection{Risk Characterization Best Practice Criteria}

5.1 Brings together hazard, exposure and dose-response assessment to make risk estimates

(quantification) and evaluation of the potential health risks from exposure to chemicals 1235611151722 28293234354247

5.2 Identifies the dominant contaminants that may potentially affect receptors (i.e. PCDD/DF, cadmium, cobalt, PCBs, $\mathrm{SO}_{2}$, mercury, lead, arsenic) 212223363

5.3 Identifies the exposure scenario(s) and risk analysis associated with them 1212223132

5.3.1 Evaluates the risks specific to the general population and the different sub-populations

(i.e. vulnerable life-stages) and put them into perspective by comparing them to other risks ${ }^{12}$

361112162135394041424347

5.4 Calculates a hazard quotient for non-carcinogens through various exposure pathways 1251112131522 27283132354463

5.4.1 Calculates a hazard index reflecting exposure to mixture of chemicals though multiple exposure pathways 21112182225283235

5.5 Excess lifetime cancer risk calculated are compared to a de minimis level ( 1 in a million/ 1 in one hundred thousand) 211121315202224252728293132343562

5.6 Identifies any exceedances of hazard quotient or excess life time cancer risk 215226163

5.7 Describes whether risk estimations are in accordance with Canadian or international HHRA guidelines for chemicals 2512

5.8 Accounts for assumptions and various uncertainties (i.e. extrapolations, use of adjustment factors used, models) 3571214323442475063

5.9 Assess TRVs to ensure their accuracy and that sensitive populations are adequately protected (including pregnant women and fetuses) even if TRVs are assumed to account for susceptible populations 16353743

5.10 Presents the strengths and/or limitations of the data and analyses 134424750

5.10.1 Discusses the degree of confidence in the assessment ${ }^{35283455}$

5.11 Mentions/practices the precautionary approach 51621404142434962

5.12 Discusses critical data gaps and research needs 34250

5.1

5.2

5.3

5.3.1

5.4
5.4.1

5.5

5.6

5.7

5.8
5.9

5.9.1

5.10

5.11

5.12

Summarize the compliances, deficiencies and absences present in this section and determine whether the risk characterization stage was completed sufficiently. 


\section{References Cited:}

Allsopp, M., Costner, P \& Johnston, P .2001. Incineration and human health: State of knowledge of the impacts of waste incinerators on human health (executive summary). Environmental Science and Pollution Research, 8(2), 141-145.

Agency for Toxic Substances and Disease Registry (ATSDR). n.d. Dioxins and furans. Retrieved March 8, 2014 from http://www.epa.gov/osw/hazard/wastemin/minimize/factshts/dioxfura.pdf

Agency for Toxic Substances and Disease Registry (ATSDR). 1995. Polycyclic aromatic hydrocarbons (PAHs)-ToxFAQs. Retrieved March 16, 2014 from http://www.atsdr.cdc.gov/toxfaqs/tfacts69.pdf

Agency for Toxic Substances and Disease Registry (ATSDR). 1999. Toxicological profile for mercury.

Agency for Toxic Substances and Disease Registry (ATSDR). 2000. Polychlorinated biphenyls-ToxFAQs. Retrieved March 11, 2014 from http://www.atsdr.cdc.gov/toxfaqs/tfacts17.pdf

Agency for Toxic Substances and Disease Registry (ATSDR).2002a. ToxFAQs for beryllium. Retrieved March 15, 2014 from http://www.atsdr.cdc.gov/toxfaqs/tf.asp?id=184\&tid=33

Agency for Toxic Substances and Disease Registry (ATSDR). 2002b. ToxFAQs for hydrogen chloride. Retrieved March 11, 2014 from http://www.atsdr.cdc.gov/toxfaqs/tf.asp?id=759\&tid=147

Agency for Toxic Substances and Disease Registry (ATSDR). 2003. ToxFAQs for hydrogen fluoride. Retrieved March 11, 2014 from http://www.atsdr.cdc.gov/toxfaqs/tf.asp?id=211\&tid=38

Agency for Toxic Substances and Disease Registry (ATSDR). 2004. ToxFAQ for ammonia. Retrieved March 11, 2014 from http://www.atsdr.cdc.gov/toxfaqs/tfacts126.pdf 
Agency for Toxic Substances and Disease Registry (ATSDR). 2007. ToxFAQs for arsenic.

Retrieved March 9, 2014 from http://www.atsdr.cdc.gov/toxfaqs/tfacts2.pdf

Agency for Toxic Substances and Disease Registry (ATSDR). 2008. ToxFAQs for chromium. Retrieved March 14, 2014 from http://www.atsdr.cdc.gov/tfacts7.pdf

Architecture, Engineering, Consulting, Operations and Maintenance (AECOM). 2010. Human health risk assessment for the capacity optimization project: Hennepin energy resource company Minneapolis, Minnesota: AECOM.

Ashworth, D. C., Elliott, P., \& Toledano, M. B. 2014. Waste incineration and adverse birth and neonatal outcomes: A systematic review. Environment International, 69, 120-132.

Balls, M., Amcoff, P., Bremer, S., Casati, S., Coecke, S., Clothier, R., Zuang, V. 2006. The Principles of Weight of Evidence Validation of Test Methods and Testing Strategies: The Report and Recommendations of ECVAM Workshop 58. Alternatives to Laboratory Animals : ATLA, 34(6), 603-620.

Batterman, S. 2004. Findings on an assessment of small scale incinerators for health-care waste. Geneva: WHO. pp.27

Borja-Aburto, V. H., Hertz-Picciotto, I., Lopez, M. R., Farias, P., Rios, C., \& Blanco, J. (1999). Blood lead levels measured prospectively and risk of spontaneous abortion. American Journal of Epidemiology, 150(6), 590-597.

Brown, R. C., Barone, J., Stanley, \& Kimmel, C. A. 2008. Children's health risk assessment: Incorporating a lifestage approach into the risk assessment process. Birth Defects Research. Part B, Developmental and Reproductive Toxicology, 83(6), 511-521.

Calow, P. (1998). Handbook of environmental risk assessment and management. University of Sheffield, UK: Wiley 
Canadian Environmental Law Agency (CELA). 2000. Risk assessment and the precautionary principle in Environmental standard setting and children's health (pp.61). CELA.

Candela, S., Ranzi, A., Bonvicini, L., Baldacchini, F., Marzaroli, P., Evangelista, A., \& Forastiere, F. 2013. Air pollution from incinerators and reproductive outcomes: a multisite study. Epidemiology, 24(6), 863-870

Candela, S., Evangelista, A., Marzaroli, P., Giorgi Rossi, P., Forastiere, F., Bonvicini, L.., \& Angelini, P. 2015. Exposure to emissions from municipal solid waste incinerators and miscarriages: A multisite study of the MONITER project.Environment International, 78, $51-60$

Cangialosi, F., Intini, G., Liberti, L., Notarnicola, M., \& Stellacci, P. 2008. Health risk assessment of air emissions from a municipal solid waste incineration plant - A case study. Waste Management, 28(5), 885-895.

Cantox Environmental Inc. 2000. Human health risk assessment for the proposed expansion of the kms peel, inc. Brampton, energy-from-waste facility. Brampton, ON.

Carter-Whitney, M., \& Canadian Institute for Environmental Law and Policy. 2007. Ontario's waste management challenge: Is incineration an option? pp.1-45

Chance, G. W. 2001. Environmental contaminants and children's health: Cause for concern, time for action. Paediatrics \& Child Health, 6(10), 731-743.

Clapp, R. W., Baraldi, C., Grassman, J., Mirer, F., Robie, D., \& Schnall, S. 2014. On agent orange in Vietnam. American Journal of Public Health, 104(10), 1860 
Cucchiella, F., D’Adamo, I., \& Gastaldi, M. 2014. Sustainable management of waste-to-energy facilities. Renewable and Sustainable Energy Reviews, 33, 719-728.

Cohen Hubal, E. A., Moya, J., \& Selevan, S. G. 2008. A life-stage approach to assessing children's exposure. Birth Defects Research Part B: Developmental and Reproductive Toxicology, 83(6), 522-529.

Cohen Hubal, E. A., de Wet, T., Du Toit, L., Firestone, M. P., Ruchirawat, M., van Engelen, J., \& Vickers, C. 2014. Identifying important life-stages for monitoring and assessing risks from exposures to environmental contaminants: Results of a world health organization review. Regulatory Toxicology and Pharmacology : RTP, 69(1), 113.

Commonwealth of Massachusetts Department of Environmental Protection. (2008). Administrative Consent order with penalty and notice of noncompliance. Retrieved October 15,2013 from http://www.covantawatch.org/p/violations-over-years.html

Commonwealth Pennsylvania Department of Environmental Protection. (2009).Consent assessment of civil penalty. Retrieved October 15, 2013 from http://www.covantawatch.org/p/violations-over-years.html

Covello, V.T and Mumpower, J. 1985. Risk analysis and risk management: A historical perspective. Risk Anal.5, 103-120.

CPF Associates. 2005. Human health and ecological impact analysis of the Hillsborough county resource recovery facility expansion. Takoma Park: Maryland.

Daston, G., E. Faustman, G. Ginsberg, P. Fenner-Crisp , S. Olin, B. Sonawane and T.J McLaughlin. 2004. A framework for assessing risks to children from exposure to environmental agents. Environmental Health Perspectives, 112(2), 238-256. 
Dejmek, J., Selevan, S.G., Benes, I. Solanský, I \& Srám.R.J. 1999. Fetal growth and maternal exposure to particulate matter during pregnancy. Environmental Health Perspectives, 107(6), 475-480.

Demidova, O., A. Cherp. 2005. Risk assessment for improved treatment of health considerations in EIA. Environmental Impact Assessment Review, 25(4), 411-429.

Diav-Citrin, O. 2011. Prenatal exposures associated with neurodevelopmental delay and disabilities. Developmental Disabilities Research Reviews, 17(2), 71-84.

Department of Energy and Environmental Protection. 2011. Settlement reached with Covanta for air emissions violations at plant in Wallingford. Retrieved October 15, 2013 from http://www.ct.gov/deep/cwp/view.asp?A=4013\&Q=483234

Durham York Waste. 2011. FAQs-How much energy is produced and what is it used for? Retrieved February 10, 2015 from http://www.durhamyorkwaste.ca/FAQ/FAQ.aspx\#howmuchenergy

Electric Power Research Institute (EPRI). 2013. Multi-pathway human health and ecological risk assessment for a model coal-fired power plant using a revised arsenic bioconcentration factor for edible fish. (Project No. 1026874). Palo Alto, CA: EPRI.

Entech-REM Canada. 2013. Environmental screening report-waste management, recycling and power generation facility.

Environment Canada. 2013a. Criteria air contaminants and related pollutants. Retrieved February 16, 2014 from https://www.ec.gc.ca/air/default.asp?lang=En\&n=7C43740B-1

Environment Canada. 2013b. Ammonia. Retrieved April 12, 2014 from https://www.ec.gc.ca/air/default.asp?lang=En\&n=27A52116-1 
Environmental Services Committee. 2013. Durham-York energy centre construction project update.

Farlie,I. 2007. Tritium hazard report: pollution and radiation risk from Canadian nuclear facilities. Toronto: Greenpeace Canada, June.

Felter, S. P., Daston, G. P., Euling, S. Y., Piersma, A. H., \& Tassinari, M. S. (2015).

Assessment of health risks resulting from early-life exposures: Are current chemical toxicity testing protocols and risk assessment methods adequate? Critical Reviews in Toxicology, 45(3), 219-244.

Frías, J. L., \& Gilbert-Barness, E. 2008. Human teratogens: Current controversies. Advances in Pediatrics, 55(1), $171-211$.

Ginsberg, G., Slikker, W., Bruckner, J., \& Sonawane, B. 2004. Incorporating children's toxicokinetics into a risk framework. Environmental Health Perspectives, 112(2), 272283.

Giroux Environmental Consulting.2014. State of waste management in Canada. Ontario, Submitted to the CCME.

Government of Alberta. 2011. Guidance on human health risk assessment for environmental impact assessment in Alberta. Alberta Health and Wellness

Guest, G., Bunce, A. \& Johnson, L .2006. How many interviews are enough? An experiment with data saturation and variability. Field Methods. 18 (5).

Harada, M. 1995. Minamata disease: Methylmercury poisoning in Japan caused by environmental pollution. Critical Reviews in Toxicology, 25(1), 1-24. 
Health Canada. 2005a. Dioxins and furans. Retrieved March 8, 2014 from

http://www.hc-sc.gc.ca/hl-vs/iyh-vsv/environ/dioxin-eng.php

Health Canada.2005b. PCBs. Retrieved March 18, 2014 from http://www.hc-sc.gc.ca/hl-vs/iyhvsv/environ/pcb-bpc-eng.php\#he

Health Canada. 2008a. Arsenic. Retrieved March 9, 2014 from http://www.hc-sc.gc.ca/fnan/securit/chem-chim/environ/arsenic-eng.php

Health Canada. 2008b. Cadmium. Retrieved March 8, 2014 from http://www.hc-sc.gc.ca/ewhsemt/pubs/water-eau/cadmium/index-eng.php\#Health

Health Canada. 2010. Part II: Health Canada toxicological reference values (trvs) and chemical-specific factors, version 2.0. Ottawa, ON: Canada. Contaminated Sites Division, Safe Environments Directorate.

Health Canada.2011. Environmental contaminants- vulnerable populations. Retrieved May 12, 2014 from http://www.hc-sc.gc.ca/ewh-semt/contaminants/vulnerable/index-eng.php

Health Canada.2012. Federal contaminated site risk assessment in Canada, Part I. Guidance on human health preliminary quantitative risk assessment (pqra), version 2.0. Ottawa, ON: Publications, Health Canada.

Health Protection Agency. (2012). Toxicological overview - Lead.

Health Protection Scotland. 2009. Incineration of Waste and Reported Human Health Effects. Glasgow.

Holmes, L. B. 2011. Human teratogens: Update 2010. Birth Defects Research. Part A, Clinical and Molecular Teratology,91(1), 1. 
Howd, R. A. 2010. Considering changes in exposure and sensitivity in an early life cumulative risk assessment. International Journal of Toxicology, 29(1), 71-77.

Hu, H., Liu, H., Chen, J., Li, A., Yao, H., Low, F., \& Zhang, L. 2015. Speciation transformation of arsenic During municipal solid waste incineration. Proceedings of the Combustion Institute, 35(3), 28832890.

Huai, G.U.A.N., Feng-Yuan, P.I.A.O., Xiao-Wei, L.I., Qiu-Juan, L. I., Lei, X. U., \& Yokoyama, K. 2010. Maternal and fetal exposure to four carcinogenic environmental metals. Biomedical and Environmental Sciences, 23(6), 458-465.

Jackson, J. 1999. Resources- not garbage municipal solid waste in Ontario. Retrieved on October 20,2013 from http://cielap.org/pdf/EnvAgenda_Resources.pdf

Jacques Whitford Limited. 2007. Generic human health and ecological risk assessment study. (No. 100949702). Ontario

Jacques Whitford Limited. 2009a. Durham-York residual waste study: Environmental assessment study document. Ontario, Submitted to the Minister of the Environment. (Project No. 100949).

Jacques Whitford Limited.2009b. Appendix D: Calpuff methodology in Air Quality Assessment Technical Study Report. (Project No. 1009497).

Jacques Whitford Limited. 2009c. Site specific human health and ecological risk assessment-technical study report (Report No. 1009497).

Jacques Whitford Limited. 2009d. Appendix H: Toxicity Profiles in Site specific human health and ecological risk assessment-technical study. (Report No. 1009497). 
Januszkiewicz, M. 2009. The Regional Municipality of Durham: Wind of change in Ontario's Municipal Solid Waste Management [PowerPoint Slides]. Retrieved on November 15, 2013 from http://www.saewa.ca/presentations/Mirkas_Presentation.pdf

Januszkiewicz, M. 2013. From idea to reality: Durham York energy centre [PowerPoint Slides].

Retrieved on January 15, 2014 from https://www.amo.on.ca/AMO-

PDFs/Events/CRST13/EFW1.aspx

Kinghorn, A., Solomon, P., \& Chan, H. M. (2007). Temporal and spatial trends of mercury in fish collected in the English-Wabigoon river system in Ontario, Canada. Science of the Total Environment, 372(2), 615-623.

Kuntz, S. W., Ricco, J. A., Hill, W. G., \& Anderko, L. (2010). Communicating methylmercury risks and fish consumption benefits to vulnerable childbearing populations. Journal of Obstetric, Gynecologic, \& Neonatal Nursing, 39(1), 118-126.

Landrigan, P. J., \& Carlson, J. E. 1995. Environmental policy and children's health. The Future of Children, 5(2), 34-52.

Landrigan, P. J., Kimmel, C., Correa, A., and Eskenazi, B. 2004. Children's health and the environment: Public health issues and challenges for risk assessment. Environmental Health Perspectives, 112(2), 257-265.

Lechuga, V. 2012. Exploring culture from a distance: The utility of telephone interviews in qualitative research. International Journal of Qualitative Studies in Education, 25(3), 251268.

Lee, N., Colley, R., Bonde, J. and Simpson, J., 1999. Reviewing the quality of environmental statements And environmental appraisals, Occasional Paper Number 55. Manchester: Department of Planning and Landscape, University of Manchester. 
Llop, S., Ballester, F., Estarlich, M., Esplugues, A., Rebagliato, M., \& Iñiguez, C. 2010. Preterm birth and exposure to air pollutants during pregnancy. Environmental Research, 110(8), 778-785.

Lonati, G., \& Zanoni, F. 2012. Probabilistic health risk assessment of carcinogenic emissions from a MSW gasification plant. Environment International, 44, 80.

MacViro Consultants Inc. and Jacques Whitford Limited. 2005. Residual waste disposal planning study background document 2-5 relevant policies and approval requirements: Development of environmental assessment terms of reference. Ontario.

Makris, S. L., Thompson, C. M., Euling, S. Y., Selevan, S. G., \& Sonawane, B. 2008. A lifestage-specific approach to hazard and dose-response characterization for children's health risk assessment. Birth Defects Research. Part B, Developmental and Reproductive Toxicology, 83(6), 530-546.

McClenaghan, T., Cooper, K., Vanderlinden, L., Muldoon, P., Abelsohn, A., Khatter, K., \& Keenan, K. 2003. Environmental Standard Setting and Children's Health in Canada: Injecting precaution into risk assessment. Journal of Environmental Law and Practice, 12(2), 141-279.

McKay, G. 2002. Dioxin characterisation, formation and minimisation during municipal solid waste (MSW) incineration: Review. Chemical Engineering Journal, 86(3), 343-368.

Miller, D. 2012. Expert voices. In S.E. Baker \& R. Edwards. How many qualitative interviews is enough. National Centre for Research Methods Review Discussion Paper. (pp 31). Retrieved Dec 5, 2014 from http://eprints.ncrm.ac.uk/2273

Mueller, W. School of Graduate Studies.Program of Environmental Applied Science and Management. (2010). A critical review of health impact assessments in Ontario's nuclear industry. 
National Academy of Sciences. 1993. Pesticides in the diets of infants and children. Washington, DC: National Academies Press. pp 1-372.

National Research Council. (NRC). 1983. Risk assessment in the federal government: Managing the process (1983). Washington, D. C: National Academy Press. pp 1-205.

National Research Council (NRC).1996. Committee on risk assessment of hazardous air pollutants. Science and judgment in risk assessment

National Research Council (NRC). 2000. Committee on health effects of waste incineration. 2000. Waste incineration \& public health (pp. 1-335). National Academies Press.

National Resources Defense Council (NRDC). 2013. The story of silent spring. Retrieved Dec 15, 2013, from http://www.nrdc.org/health/pesticides/hcarson.asp

Northeast Maryland Waste Disposal Authority. 2014. Fredrick/carol country renewable waste to energy facility in Frederick county Maryland human health risk assessment with transmittals. Retrieved Jan 5, 2014 from http://www.nmwda.org/projects_and_services/documents/ FCCRWTEHumanHealthRiskAssessmentwithTransmittals.pdf

Olin, S. S., \& Sonawane, B.R. 2003. Workshop to develop a framework for assessing risks to children from exposure to environmental agents. Environmental Health Perspectives, 111(12), 1524- 1526.

Ollson, C. A., Knopper, L. D., Whitfield Aslund, M. L., \& Jayasinghe, R. 2014. Site specific risk assessment of an energy-from-waste thermal treatment facility in Durham region, Ontario, Canada. part A: Human health risk assessment. The Science of the Total Environment, 466-467, 345-356. 
Oken E, Bellinger DC. 2008. Fish consumption, methylmercury and child neurodevelopment. Current opinion in pediatrics. 20:178-83.

Ontario Ministry of the Environment (MOE). 1999. Environmental Risks of Municipal Non-Hazardous Waste Landfilling and Incineration (Technical Report Summary). Ontario: MOE, Standards Development Branch.

Ontario Ministry of the Environment. 2005. Procedures for the use of risk assessment under part XV.1 of the environmental protection act. (PIBs 5404e). Ontario.

Ontario Ministry of the Environment(MOE).2010. Protocol for updating certificates of approval for air emissions. Ontario: Environmental Assessment and Approvals Branch, MOE

Ontario Ministry of the Environment (MOE).2014a. Consultation in Ontario's Environmental Assessment Process. (PIBS 5016e01). Ontario: Environmental Approvals Access and Service Integration Branch, MOE.

Ontario Ministry of the Environment(MOE).2014b. Durham/York Residual Waste Study. Retrieved August 12, 2014 from http://www.ontario.ca/environment-and-energy/durhamyork-residualwaste-study

Ontario Ministry of the Environment (MOE). 2015. Preparing environmental assessments. Retrieved February 12, 2015 from https://www.ontario.ca/environment-and-energy/preparingenvironmental-assessments

Palmer, M. G. (2007). The case of agent orange.Contemporary Southeast Asia, 29(1), 172-195.

Patterson, J., Meek, M.E, Strawson, J.E \& Liteplo, R.G. 2007. Engaging expert peers in the development of risk assessments. Risk Analysis, 27(6), 1609-1621. 
Paustenbach, D. J. 1989. The risk assessment of environmental and human health hazards: A textbook of case studies. John Wiley \& Sons Inc.

Peel Energy Recovery. 2015. Retrieved March 3, 2015 from http://www.peelenergyrecovery.ca/get-info/project-details/

Pelekis, M., Nicolich, M. J., \& Gauthier, J. S. (2003). Probabilistic framework for the estimation of the adult and child toxicokinetic intraspecies uncertainty factors. Risk Analysis, 23(6), 1239-1255.

Pushchak, R., per comm., 2013.

Qu, S. Q., \& Dumay, J. 2011. The qualitative research interview. Qualitative Research in Accounting \& Management, 8(3), 238-264.

Ratislavová, K., \& Ratislav, J. 2014. Asynchronous email interview as a qualitative research method in the humanities. Human Affairs, 24(4), 452-460.

Rodier, P. M. 1995. Developing brain as a target of toxicity. Environmental Health Perspectives, 103 Suppl 6, 73-76.

Rubin, H. J., \& Rubin, I. (2004). Qualitative interviewing: The art of hearing data.

Sabbas, T., Speiser, C., Heuss-Assbichler, S., Klein, R., Lechner, P., and Polettini, A. 2003. Management of municipal solid waste incineration residues. Waste Management, 23(1), 61-88.

Selevan, S. G., Kimmel, C.A., \& Mendola, P. 2000. Identifying critical windows of exposure for children's health. Environmental Health Perspectives, 108(s3), 451-455.

Schulte, P. 2003. Challenges for risk assessors. Human and Ecological Risk Assessment, 9(1), 439455. 
Seidman, I. 2006. A guide for researchers in education and the social sciences. New York, Teacher's College Press.

Shah, P. S., Balkhair, T., \& Knowledge Synthesis Group on Determinants of Preterm/LBW births. 2011. Air pollution

and birth outcomes: A systematic review. Environment International,37(2), 498-516.

Smith, Lesbia and Wilson, Ross. 2009. Peer review of the draft jw shhra technical study report; DurhamYork residual waste study. Retrieved March 20, 2015 from http://archives.york.ca/councilcommitteearchives/pdf/sep\%2016\%20madill\%20energy.pdf

Snary, C. 2002. Health risk assessment for planned waste incinerators: Getting the right science and the science right. Risk Analysis : An Official Publication of the Society for Risk Analysis, 22(6), 1095-1105.

Statistics Canada. 2008. Lead, mercury and cadmium levels in Canadians. (Catalogue No. 82003-XPE). Health Reports. 19(4).

Statistics Canada. 2012. Solid waste. Retrieved January 15, 2014 from http://www.statcan.gc.ca/pub/16-201-x/2012000/part-partie3-eng.htm

Storey, K., Noble, B. F. 2004. Towards best practice EA follow-up. A Report Prepared under Contribution Agreement with the Canadian Environmental Assessment Agency Research and Development Program. Hull, Quebec: CEAA.

Sturges, J. E., \& Hanrahan, K. J. 2004. Comparing telephone and face-to-face qualitative interviewing: A research note.Qualitative Research, 4(1), 107-118. 
Takahashi, F., A. Kida \& T. Shimaoka 2010. Statistical estimate of mercury removal efficiencies for air pollution control devices of municipal solid waste incinerators. The Science of the Total Environment, 408(22), 5472-5477.

Takaoka, S., Fujino, T., Hotta, N., Ueda, K., Hanada, M., Tajiri, M., \& Inoue, Y. (2014). Signs and symptoms of methylmercury contamination in a first nations community in northwestern Ontario, Canada. The Science of The Total Environment, 468-469, 950-957.

Tango, T., Fujita, T., Tanihata, T., Minowa, M., Doi, Y., Kato, N.,\& Uehata, T. 2004. Risk of adverse reproductive outcomes associated with proximity to municipal solid waste incinerators with high dioxin emission levels in Japan.Journal of epidemiology, 14(3), 83-93.

Thompson, J. 2005. The health effects of waste incinerators. Journal of Nutritional and Environmental Medicine, 15(2-3), 115-156.

Thompson, J. \& H. Anthony, H. 2008. The health effects of waste incinerators. British Society of Ecological Medicine.

United States Department of Veteran Affairs. 2015. Agent orange. Retrieved April 29, 2015 from http://www.publichealth.va.gov/exposures/agentorange/

United States Environmental Protection Agency (USEPA). 1989. Risk assessment guidance for superfund. (No. 1). Retrieved November 15, 2013 from http://www.epa.gov/oswer/riskassessment/ragsa/pdf/rags_a.pdf

United States Environmental Protection Agency (USEPA). 2000. Beryllium compounds. Retrieved March 14, 2014 from http://www.epa.gov/ttnatw01/hlthef/berylliu.html

United States Environmental Protection Agency (USEPA). 2002. Child-specific exposure factors handbook. EPA/600/P-00/002B .Washington, DC: Office of Research and Development. 
United States Environmental Protection Agency (USEPA). 2005a.Guidelines for carcinogenic risk assessment. (EPA/630/P-03/001F) .Washington, DC: US Environmental Protection Agency.

United States Environmental Protection Agency (USEPA). 2005b. Human health risk assessment protocol (hhrap) for hazardous waste combustion facilities (final). (EPA/530/R-05/006). Office of Solid Waste, USEPA.

United States Environmental Protection Agency (USEPA). 2006. A framework for assessing health risk of environmental exposures to children (Final). (EPA/600/R-05/093F). Washington, DC: U.S. Environmental Protection Agency.

United States Environmental Protection Agency (USEPA). 2008a. Polycyclic aromatic hydrocarbons (PAHs). Retrieved March 15, 2014 from http://www.epa.gov/osw/hazard/wastemin/minimize/factshts/pahs.pdf

United States Environmental Protection Agency (USEPA). 2008b. Technical guidance manual. Retrieved November 8, 2014 from http://www.epa.gov/reg3hscd/risk/human/info/solabsg2.htm United States Environmental Protection Agency (USEPA). 2010. Human and ecological risk assessment of coal combustion wastes (draft).

United States Environmental Protection Agency (USEPA). 2011a.Exposure factors: 2011 edition. (EPA/600/R-090/052F). Washington, DC: Office of Research and Development.

United States Environmental Protection Agency (USEPA).2011b. 2005 National-scale air toxic assessment- Glossary of terms. Retrieved January 11, 2015 from http://www.epa.gov/airtoxics/natamain/gloss1.html 
United States Environmental Protection Agency.2012a. Human health risk assessment. Retrieved Oct 15, 2013, from http://www.epa.gov/risk/health-risk.htm

United States Environmental Protection Agency ( USEPA). 2012b. Step 1: Hazard identification. Retrieved September 20, 2014 from http://www.epa.gov/risk_assessment/hazardous-identification.htm

United States Environmental Protection Agency (USEPA). 2013a. Arsenic in drinking water. Retrieved March 9, 2014 from http://water.epa.gov/lawsregs/rulesregs/sdwa/arsenic/index.cfm United States Environmental Protection Agency (USEPA). 2013b. Chromium compounds. Retrieved March 18, 2014 from http://www.epa.gov/ttnatw01/hlthef/chromium.html

United States Environmental Protection Agency (USEPA). 2014a. Sulfur dioxide-health. Retrieved March 12, 2014 from http://www.epa.gov/airquality/sulfurdioxide/health.html

United States Environmental Protection Agency (USEPA). 2014b. Nitrogen dioxide-health. Retrieved March 12, 2014 from http://www.epa.gov/airquality/nitrogenoxides/health.html

United States Environmental Protection Agency. 2014c. Particulate matter-health. Retrieved March 12, 2014 from http://www.epa.gov/airquality/particlepollution/health.html

United States Environmental Protection Agency (USEPA). 2014d. Carbon monoxide-Health. Retrieved March 12, 2014 from http://www.epa.gov/airquality/carbonmonoxide/health.html

United States Environmental Protection Agency (USEPA). 2014e. Framework for human health risk assessment to inform decision making. (EPA/100/R-14/001). Office of the Science Advisor Risk Assessment Forum, U.S Environmental Protection Agency.

United States Environmental Protection Agency (USEPA). 2014f. Child-specific exposure scenarios examples. (EPA/600/R-14/217F) .Washington, DC: Office of Research and Development. 
United States Food and Drug Administration (US FDA). 2005. Reviewers guidance. Evaluating the risks of drug exposure in human pregnancies. Retrieved November 15, 2013 from http://www.fda.gov/downloads/Drugs/.../Guidances/ucm071645.pdf

Vinceti, M., Malagoli, C., Teggi, S., Fabbi, S., Goldoni, C., De Girolamo, G.,\& Bergomi, M. 2008. Adverse pregnancy outcomes in a population exposed to the emissions of a municipal waste incinerator. Science of the Total Environment,407(1), 116-121.

Wilson Scientific Consulting Inc. (2013). Human health risk assessment of the mercury from the proposed keeyask generation project. Retrieved March 20, 2014 from http://keeyask.com/wp/wpcontent/uploads/2012/07/03-Human-Health-Risk-Cd-version.pdf

World Bank 1997. Environmental hazard and risk assessment. Environmental assessment sourcebook. Washington.

World Health Organization (WHO). 2006. Principles for evaluating health risks in children associated with exposure to chemicals. Germany: WHO Library.

World Health Organization (WHO). 2008. Children are not little adults. Retrieved Oct 2013, 2013, from http://www.who.int/ceh/capacity/Children_are_not_little_adults.pdf

World Health Organization (WHO). 2010. WHO human health risk assessment toolkit: Chemical hazards. (Project Document No. 8). WHO Library.

World Health Organization(WHO). 2014. Dioxins and their effects of human health. Retrieved March 8, 2014 from http://www.who.int/mediacentre/factsheets/fs225/en/ 
York Region. 2011. Durham York energy from waste project update. Retrieved April 19, 2013, from http://www.york.ca/NR/rdonlyres/fr6p6rpijgkizioko4yfq2xacyuy4sojkcnbpvt7ramp34io psq2erha p2b6zaohd4p6u4nrlna2abxma3o5i576b/rpt+1+cls +5 . pdf

Zhu, X., Liu, Y., Chen, Y., Yao, C., Che, Z., \& Cao, J. 2015. Maternal exposure to fine particulate matter (PM2.5) and pregnancy outcomes: A meta-analysis. Environmental Science and Pollution Research, 22(5), 3383-3396. 
Endnotes:

${ }^{1}$ United States Environmental Protection Agency (USEPA). 2005a.Guidelines for carcinogenic risk assessment. (EPA/630/P-03/001F) .Washington, DC: US Environmental Protection Agency.

${ }^{2}$ Cantox Environmental Inc. 2000. Human health risk assessment for the proposed expansion of the kms peel, inc. Brampton, energy-from-waste facility. Brampton, ON.

${ }^{3}$ Daston, G., E. Faustman, G. Ginsberg, P. Fenner-Crisp , S. Olin, B. Sonawane and T.J McLaughlin. 2004. A framework for assessing risks to children from exposure to environmental agents. Environmental Health Perspectives, 112(2), 238-256.

${ }^{4}$ Landrigan, P. J., Kimmel, C., Correa, A., and Eskenazi, B. 2004. Children's health and the environment: Public health issues and challenges for risk assessment. Environmental Health Perspectives, 112(2), 257-265.

${ }^{5}$ Government of Alberta. 2011. Guidance on human health risk assessment for environmental impact assessment in Alberta. Alberta Health and Wellness

${ }^{6}$ United States Environmental Protection Agency. 1992. EPA's approach for assessing the risks associated with chronic exposures to carcinogens. Retrieved Jan 12, 2014 from http://www.epa.gov/iris/carcino.htm

${ }^{7}$ United States Environmental Protection Agency (USEPA). 2014. Framework for human health risk assessment to inform decision making. (EPA/100/R-14/001). Office of the Science Advisor Risk Assessment Forum, U.S Environmental Protection Agency.

${ }^{8}$ Wheatley, Andrew and Sadhra, Steven.2010.Carcinogenic risk assessment for emissions from clinical waste incineration and road traffic. International journal of environmental health research 20.5, 313-327.

${ }^{9}$ Boudet, C., Zmirou, D., Laffond, M., Balducci, F., \& Benoit-Guyod, J. 1999. Health risk assessment of a modern municipal waste incinerator. Risk Analysis, 19(6), 1215-1222

${ }^{10}$ Lee, N., Colley, R., Bonde, J. and Simpson, J., 1999. Reviewing the quality of environmental statements and environmental appraisals, Occasional Paper Number 55. Manchester: Department of Planning and Landscape, University of Manchester. 
${ }^{11}$ United States Environmental Protection Agency (USEPA). 2005b. Human health risk assessment protocol (hhrap) for hazardous waste combustion facilities (final). (EPA/530/R-05/006). Office of Solid Waste, USEPA.

${ }^{12}$ Northeast Maryland Waste Disposal Authority. 2014. Fredrick/carol country renewable waste to energy facility in Frederick county Maryland human health risk assessment with transmittals. Retrieved Jan 5, 2014 from http://www.nmwda.org/projects_and_services/documents/ FCCRWTEHumanHealthRiskAssessmentwithTransmittals.pdf

${ }^{13}$ Cangialosi, F., Intini, G., Liberti, L., Notarnicola, M., \& Stellacci, P. 2008. Health risk assessment of air emissions from a municipal solid waste incineration plant - A case study. Waste Management, 28(5), 885-895.

${ }^{14}$ Kao, W., Ma, H., Wang, L., \& Chang-Chien, G. 2007. Site-specific health risk assessment of dioxins and furans in an industrial region with numerous emission sources. Journal of Hazardous Materials, 145(3), 471-481.

${ }^{15} \mathrm{Hashm}$, Ahlim. 2008. Human health risk assessment/ technical appendix energy from waste facility, Oxwellmains Viridor waste management Itd. (environmental statement). (SAW0602/N/DRH). RPS Planning \& Development.

${ }^{16}$ Interviewee A per. comm., 2014

${ }^{17}$ National Research Council (NRC).1996. Committee on risk assessment of hazardous air pollutants. Science and judgment in risk assessment

${ }^{18}$ Calow, P. (1998). Handbook of environmental risk assessment and management. University of Sheffield, UK: Wiley

${ }^{19}$ Cocarta, D. M., Rada, E. C., Ragazzi, M., Badea, A., \& Apostol, T. 2009. A contribution for a correct vision of health impact from municipal solid waste treatments. Environmental technology, 30(9), 963-968.

${ }^{20}$ Rovira, J., Mari, M., Nadal, M., Schuhmacher, M., \& Domingo, J. L. (2010). Environmental monitoring of metals, PCDD/Fs and PCBs as a complementary tool of biological surveillance to assess human health risks. Chemosphere, 80(10), 1183-1189.

${ }^{21}$ Interviewee C per. comm., 2014 
${ }^{22}$ Architecture, Engineering, Consulting, Operations and Maintenance (AECOM). 2010. Human health risk assessment for the capacity optimization project: Hennepin energy resource company Minneapolis, Minnesota: AECOM.

${ }^{23}$ Lonati, G., \& Zanoni, F. 2012. Probabilistic health risk assessment of carcinogenic emissions from a MSW gasification plant. Environment International, 44, 80.

${ }^{24}$ Entech-REM Canada. 2013. Environmental screening report-waste management, recycling and power generation facility.

${ }^{25}$ CPF Associates. 2005. Human health and ecological impact analysis of the Hillsborough county resource recovery facility expansion. Takoma Park: Maryland.

${ }^{26}$ Cordioli, M., Vincenzi, S., \& De Leo, G. A. 2013. Effects of heat recovery for district heating on waste incineration health impact: a simulation study in Northern Italy. Science of the Total Environment, 444, 369-380.

${ }^{27}$ Morselli, L., Passarini, F., Piccari, L., Vassura, I., \& Bernardi, E. (2010). Risk assessment applied to air emissions from a medium-sized Italian MSW incinerator. Waste Management \& Research.

${ }^{28}$ Garrick, B. J. (2002). The use of risk assessment to evaluate waste disposal facilities in the United States of America. Safety Science, 40(1), 135-151.

${ }^{29}$ Rao, R.K., Stormwind, B.L., Chaudhuri, I and Garcia, M. 2004. Multiple pathway health risk assessment an multimedia environmental monitoring programs for a municipal waste resource recovery facility in Maryland. North American Waste to Energy Conference. Savannah, Georgia.

${ }^{30}$ Ma, X. J., Jiang, X. G., Jin, Y. Q., Liu, H. M., Li, X. D., Chen, T., \& Yan, J. H. (2012). Dispersion modeling and health risk assessment of dioxin emissions from a municipal solid waste incinerator in Hangzhou, China. Journal of Zhejiang University Science A, 13(1), 69-78.

${ }^{31}$ United States Environmental Protection Agency (USEPA). 2010. Human and ecological risk assessment of coal combustion wastes (draft).

${ }^{32}$ Electric Power Research Institute (EPRI). 2013. Multi-pathway human health and ecological risk assessment for a model coal-fired power plant using a revised arsenic bioconcentration factor for edible fish. (Project No. 1026874). Palo Alto, CA: EPRI. 
${ }^{33}$ Health Canada.2012. Federal contaminated site risk assessment in Canada, Part I. Guidance on human health preliminary quantitative risk assessment (pqra), version 2.0. Ottawa, ON: Publications, Health Canada.

${ }^{34}$ Snary, C. 2002. Health risk assessment for planned waste incinerators: Getting the right science and the science right. Risk Analysis : An Official Publication of the Society for Risk Analysis, 22(6), 1095-1105.

${ }^{35}$ Ontario Ministry of the Environment. 2005. Procedures for the use of risk assessment under part XV.1 of the environmental protection act. (PIBs 5404e). Ontario.

${ }^{36}$ The Regional Municipality of Halton.2007. Step 4A: Identification and description of potential health and environmental effects. Oakville, ON.

${ }^{37}$ Health Canada. 2010. Part II: Health Canada toxicological reference values (trvs) and chemical-specific factors, version 2.0. Ottawa, ON: Canada. Contaminated Sites Division, Safe Environments Directorate.

${ }^{38}$ National Academy of Sciences. (2000). Executive Summary: Waste Incineration and Public Health. Washington, DC: Committee on Health Effects of Waste Incineration, Board on Environmental Studies and Toxicology, \& National Research Council. Retrieved from http://www.metrovancouver org/services/solidwaste/planning/ReportsforQA/NASNRC_Wastelncineration.pdf

39 Interviewee D per comm., 2014

${ }^{40}$ Interviewee B per comm., 2014

${ }^{41}$ Interviewee E per. comm., 2014

${ }^{42}$ United States Environmental Protection Agency (USEPA). 2006. A framework for assessing health risk of environmental exposures to children (final). (EPA/600/R-05/093F). Washington, DC: U.S. Environmental Protection Agency.

${ }^{43}$ Interviewee F per comm., 2014

${ }^{44}$ World Health Organization (WHO). 2010. WHO human health risk assessment toolkit: Chemical hazards. (Project Document No. 8). WHO Library.

${ }^{45}$ Olin, S. S., \& Sonawane, B.R. 2003. Workshop to develop a framework for assessing risks to children from exposure to environmental agents. Environmental Health Perspectives, 111(12), 1524- 1526. 
${ }^{46}$ Ginsberg, G., Slikker, W., Bruckner, J., \& Sonawane, B. 2004. Incorporating children's toxicokinetics into a risk framework. Environmental Health Perspectives, 112(2), 272283.

${ }^{47}$ World Health Organization (WHO). 2006. Principles for evaluating health risks in children associated with exposure to chemicals. Germany: WHO Library.

${ }^{48}$ Selevan, S. G., Kimmel, C.A., \& Mendola, P. 2000. Identifying critical windows of exposure for children's health. Environmental Health Perspectives, 108(s3), 451-455.

${ }^{49}$ McClenaghan, T., Cooper, K., Vanderlinden, L., Muldoon, P., Abelsohn, A., Khatter, K., \& Keenan, K. 2003. Environmental Standard Setting and Children's Health in Canada: Injecting precaution into risk assessment. Journal of Environmental Law and Practice, 12(2), 141-279.

${ }^{50}$ Brown, R. C., Barone, J., Stanley, \& Kimmel, C. A. 2008. Children's health risk assessment: Incorporating a lifestage approach into the risk assessment process. Birth Defects Research. Part B, Developmental and Reproductive Toxicology, 83(6), 511-521.

${ }^{51}$ Cohen Hubal, E. A., Moya, J., \& Selevan, S. G. 2008. A life-stage approach to assessing children's exposure. Birth Defects Research Part B: Developmental and Reproductive Toxicology, 83(6), 522-529.

${ }^{52}$ United States Environmental Protection Agency (USEPA). 2011. Exposure factors handbook: 2011 edition. (EPA/600/R-090/052F). Washington, DC: Office of Research and Development.

${ }^{53}$ Landrigan, P. J., \& Carlson, J. E. 1995. Environmental policy and children's health. The Future of Children, 5(2), 34-52.

${ }^{54}$ British Columbia Centre for Disease Control. 2012. Health assessment for thermal treatment of municipal solid waste in British Columbia (final).

${ }^{55}$ Demidova, O., A. Cherp. 2005 . Risk assessment for improved treatment of health considerations in EIA. Environmental Impact Assessment Review, 25(4), 411-429.

${ }^{56}$ National Research Council (NRC). 2000. Committee on health effects of waste incineration. 2000. Waste incineration \& public health (pp. 1-335). National Academies Press.

${ }^{57}$ Meneses, M., Schuhmacher, M., \& Domingo, J. L. 2004. Health risk assessment of emissions of dioxins and furans from a municipal waste incinerator: comparison with other emission sources. Environment international, 30(4), 481-489. 
${ }^{58}$ Makris, S. L., Thompson, C. M., Euling, S. Y., Selevan, S. G., \& Sonawane, B. 2008. A lifestage-specific approach to hazard and dose-response characterization for children's health risk assessment. Birth Defects Research. Part B, Developmental and Reproductive Toxicology, 83(6), 530-546.

${ }^{59}$ Pioneer Technologies Corporation. 2001. Final Spokane waste-to-energy facility human health risk assessment. Spokane, WA.

${ }^{60}$ United States Environmental Protection Agency (USEPA). 2009. Highlights of the child-specific Exposure factors handbook (EPA/600/R-08/135). Washington, DC: Office of Research and Development.

${ }^{61}$ Cameco Corporation. 2009. Port hope conversion facility site-wide risk assessment: Human health and ecological risk assessment. Port Hope, ON.

${ }^{62}$ Interviewee G per comm., 2014

${ }^{63}$ Wilson Scientific Consulting Inc. (2013). Human health risk assessment of the mercury from the proposed keeyask generation project. Retrieved March 20, 2014 from http://keeyask.com/wp/wpcontent/uploads/2012/07/03-Human-Health-Risk-Cd-version.pdf

${ }^{64}$ Felter, S. P., Daston, G. P., Euling, S. Y., Piersma, A. H., \& Tassinari, M. S. (2015).

Assessment of health risks resulting from early-life exposures: Are current chemical toxicity testing protocols and risk assessment methods adequate? Critical Reviews in Toxicology, 45(3), 219-244. 